\title{
Effective Primary
}

Pedagogical Strategies in

English and Mathematics in

Key Stage 2: A study of

Year 5 classroom practice

from the EPPSE 3-16

longitudinal study

Iram Siraj-Blatchford

Donna-Lynn Shepherd

Edward Melhuish

Brenda Taggart

Pam Sammons

Kathy Sylva

Department for

Education 
This research report was commissioned before the new UK Government took office on 11 May 2010. As a result the content may not reflect current Government policy and may make reference to the Department for Children, Schools and Families (DCSF) which has now been replaced by the Department for Education (DFE).

The views expressed in this report are the authors' and do not necessarily reflect those of the Department for Education. 


\section{THE EPPSE RESEARCH TEAM}

\section{Principal Investigators}

\section{Professor Kathy Sylva}

Department of Educational Studies, University of Oxford

0044 (0)1865 274008 / email kathy.sylva@education.ox.ac.uk

\section{Professor Edward Melhuish}

Institute for the Study of Children, Families and Social Issues

Birkbeck University of London

0044 (0)207 0790834 / email e.melhuish@bbk.ac.uk

\section{Professor Pam Sammons}

Department of Educational Studies, University of Oxford

0044 (0)1865 274142 / email pamela.sammons@education.ox.ac.uk

\section{Professor Iram Siraj-Blatchford}

Institute of Education, University of London

0044 (0)207 6126218 / email i.siraj-blatchford@ioe.ac.uk

\section{*Brenda Taggart}

Institute of Education, University of London

0044 (0)207 6126219 / email b.taggart@ioe.ac.uk

\section{Research Officers}

\section{Donna-Lynn Shepherd}

Institute of Education, University of London

0044 (0)2076126608 / email dshepherd01@ioe.ac.uk

\section{Dr Katalin Toth}

Institute of Education, University of London

0044 (0)207 9115587 / email k.toth@ioe.ac.uk

\section{Diana Draghici}

Institute of Education, University of London

0044 (0)207 6126608 / email d.draghici@ioe.ac.uk

\section{Tracking Officer}

\section{Wesley Welcomme}

Institute of Education, University of London

0044 (0)2076126684 / email w.welcomme@ioe.ac.uk

*Also Research Co-ordinator 


\title{
AUTHORS
}

\author{
Iram Siraj-Blatchford \\ Donna-Lynn Shepherd \\ Edward Melhuish \\ Brenda Taggart \\ Pam Sammons
}

Kathy Sylva

\section{ACKNOWLEDGEMENT}

The EPPE project is a major longitudinal study funded by the Department for Education. The research would not be possible without the support and co-operation of the six Local Authorities (LAs) and the many pre-school centres, primary schools, children and parents participating in the research.

We would like to give special thanks to the research assistants: Rosemary Ellis, Jill Head, Isabella Hughes, Rose Jennings, Margaret Kehoe, Laura Manni, Helen Mirelman, Stella Muttock and John Stokes who collected the data for this report.

We are also grateful to Professor John Siraj-Blatchford for his advice on the early stages of this study and the children and staff of Hamilton Primary School and their headteacher, Graeme Aldous, for helping us refine the pedagogical strategies; their feedback was very helpful. In addition we are grateful to Christopher Stone of Arthur Terry School for allowing us access to his school's policies.

The views expressed in this report are the authors' and do not necessarily reflect those of the Department for Education

@ JUNE 2011 Siraj-Blatchford, Melhuish, Taggart, Sammons \& Sylva, 
Executive Summary

Section 1 Introduction to the Effective Primary Pedagogical Strategies in English and Maths (EPPSEM) Study ................................................................ 1

1.1 Introduction to the Effective Provision of Pre-School, Primary and Secondary Education (EPPSE 3-16) Research Project (1997 - 2013)......

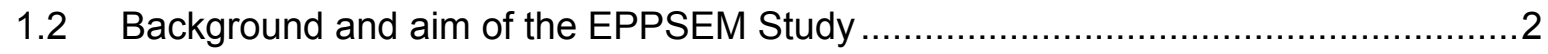

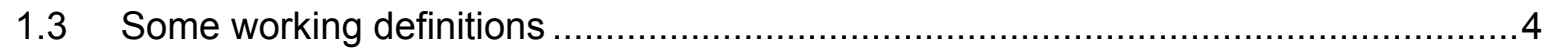

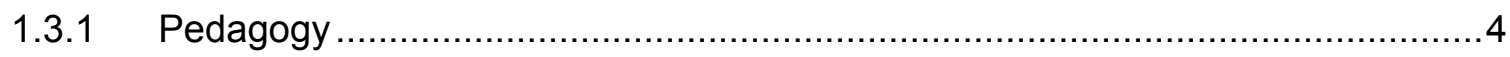

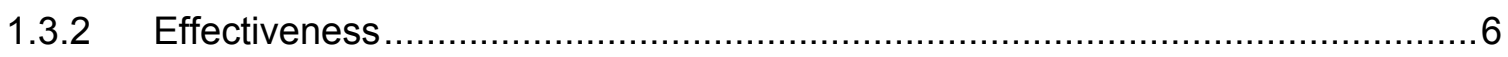

1.4 Effective pedagogical strategies: what the literature tells us..................................

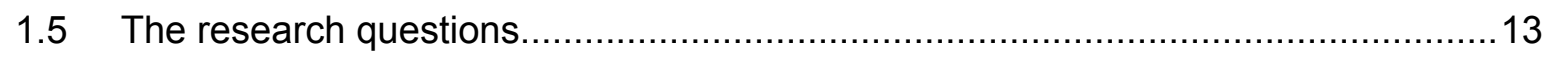

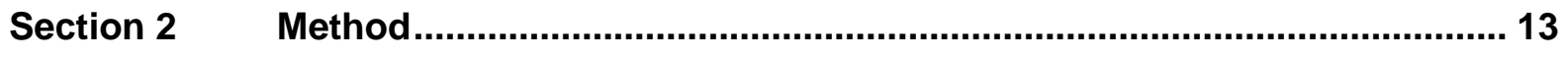

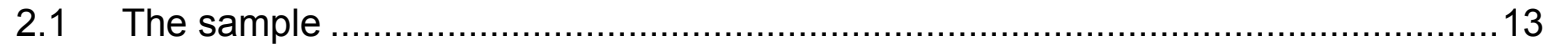

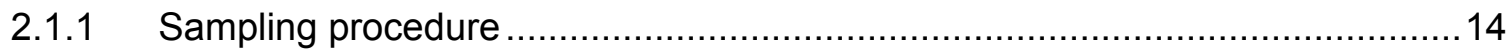

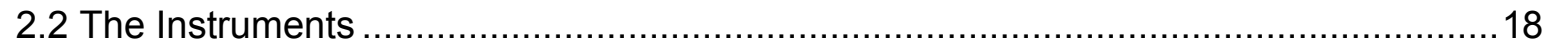

2.2.1 The Classroom Observation System for Fifth Grade (COS-5, Pianta)...................18

2.2.2 Instructional Environment Observation Scale (IEO, Stipek) ................................23

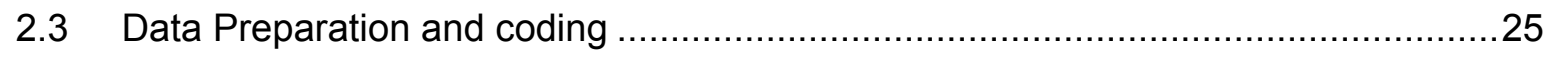

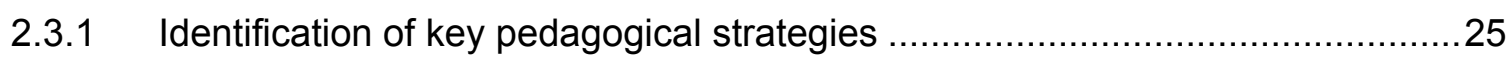

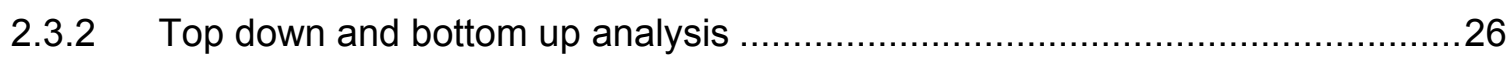

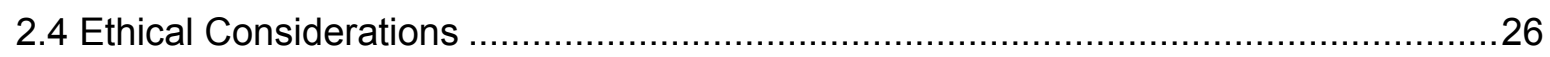

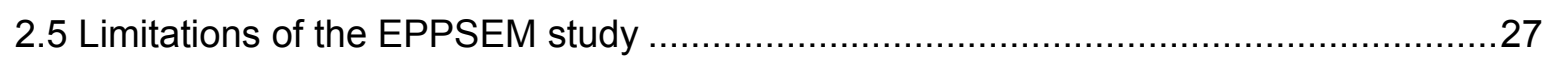

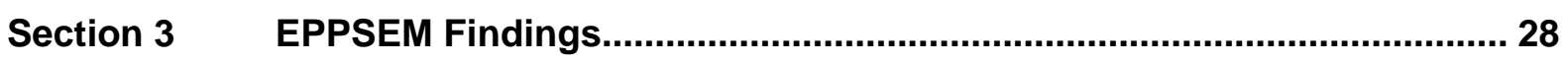

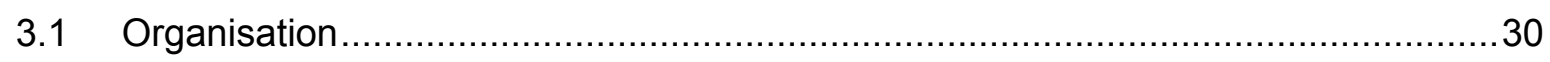

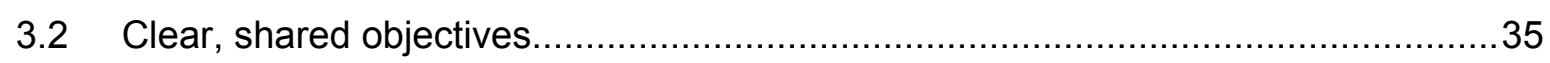

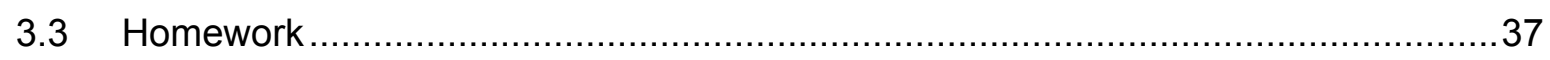

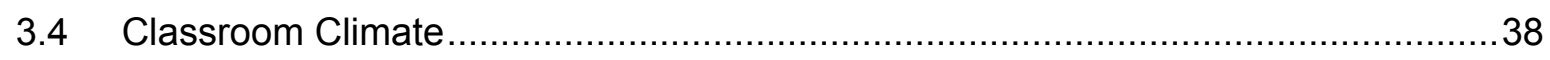

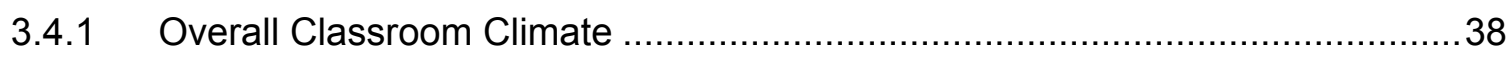

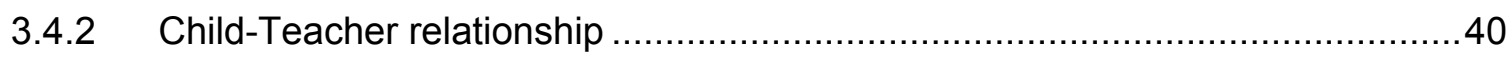

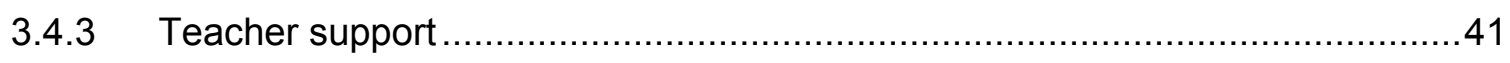

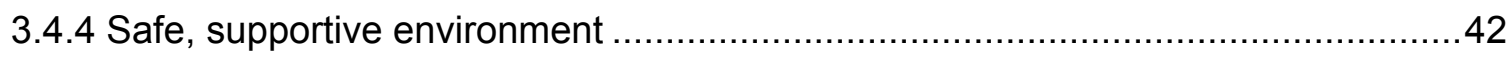

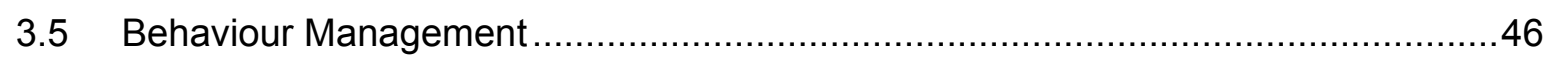

3.5.1 Frequency of disciplining and levels of disruptive behaviour ........................46 


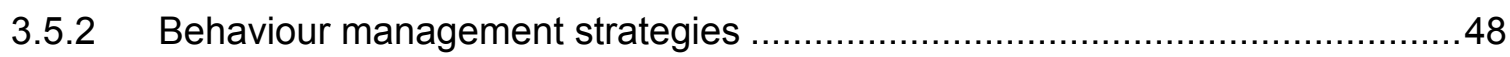

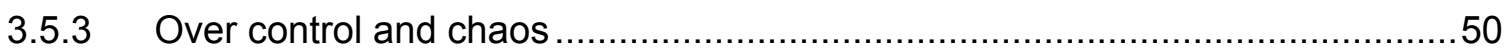

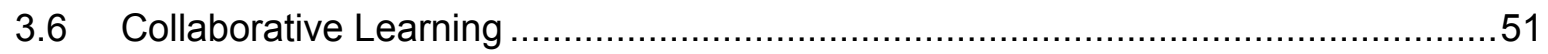

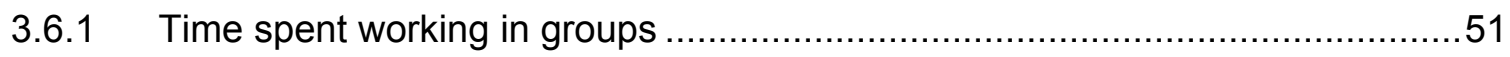

3.6.2 Use of groups for a specific, collaborative purpose ......................................53

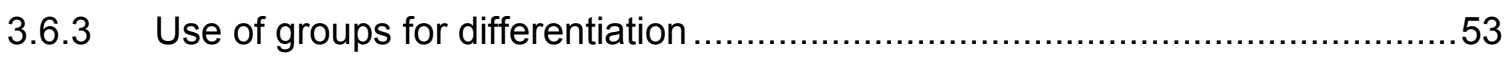

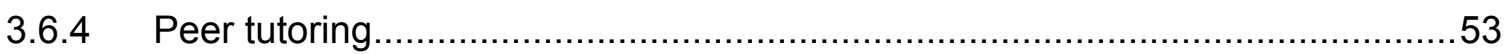

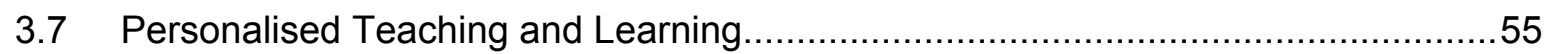

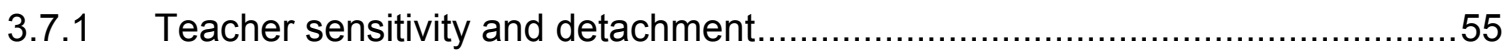

3.7.2 Variety and richness of teaching resources. ................................................56

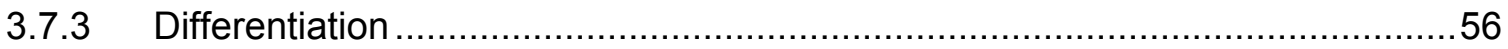

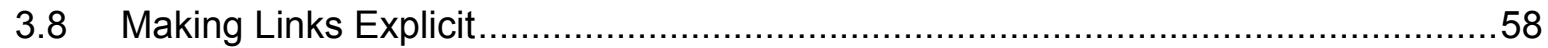

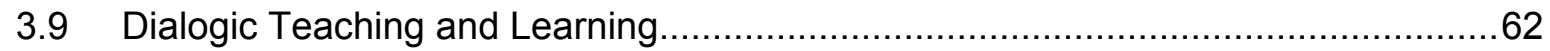

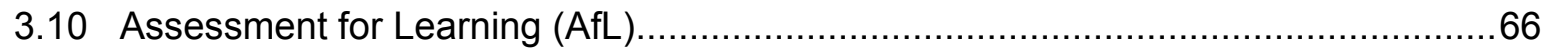

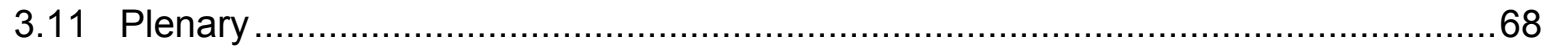

3.11.1 Informal assessment of children's understanding of basic concepts and skills 69

3.11.2 Providing an opportunity for children to share their work and receive feedback69

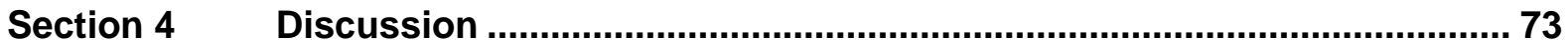

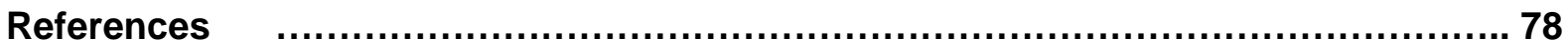

Appendix 1 The Influence of school and teaching quality on children's progress in

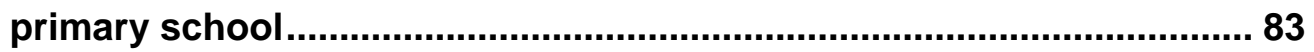

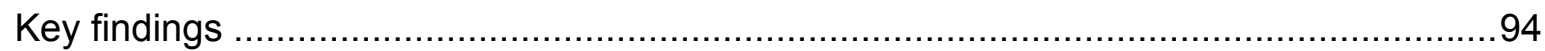

Appendix 2 The classroom observation instruments: .......................................... 101

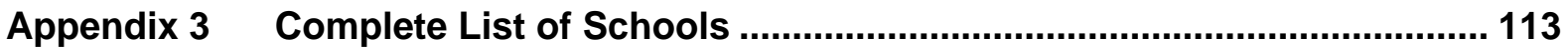

Appendix 4 IEO and COS-5: Training and Reliability ......................................... 116

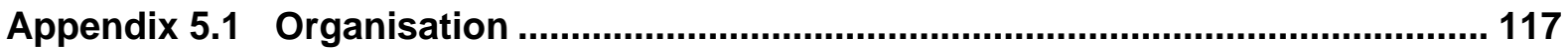

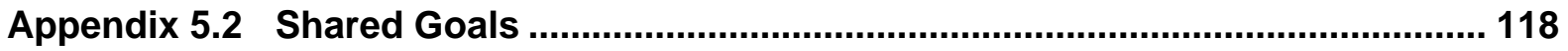

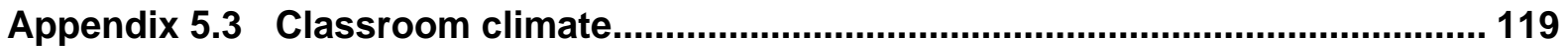

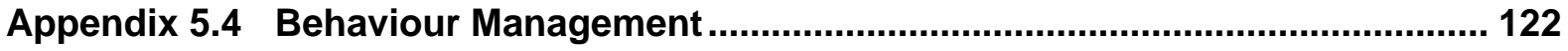

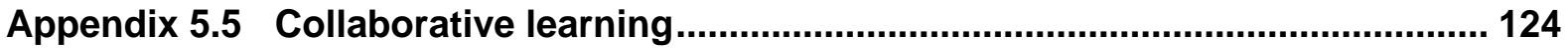

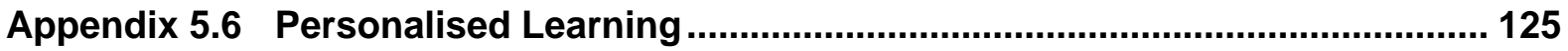

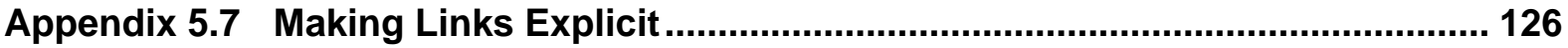

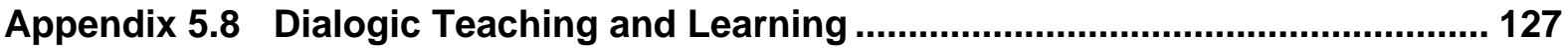

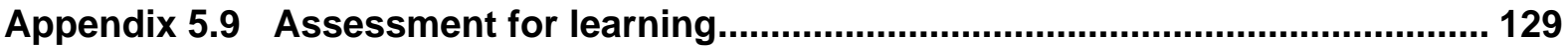

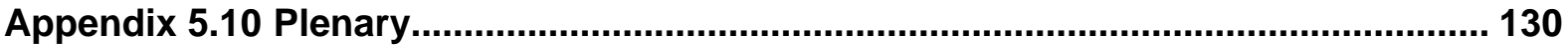




\section{List of Tables}

Table 2.1a Criteria for inclusion in Group A: Excellent schools (academically effective, good quality pedagogy)

Table $2.1 \mathrm{~b} \quad$ Characteristics of the ten typical academically effective schools with good quality pedagogy - Excellent

Table 2.1c Criteria for inclusion in Group B: Good schools (medium academic effectiveness and medium quality pedagogy)

Table 2.1d Characteristics of the nine typical schools with medium academic effectiveness and medium quality pedagogy -Good

Table 2.1e Criteria for inclusion in Group C: Poor schools (low academic effectiveness,

Table 2.1f Characteristics of the ten typical schools with low academic effectiveness and poor quality pedagogy - Poor

Table 2.2a Table 2.2a COS-5: Number of cycles as a function of number of schools

Table $2.2 b$ COS-5: Number of cycles broken down by type of lesson

Table 2.3a Effective pedagogical strategies

Qualitative descriptions of the numerical ratings Quantitative scores and their qualitative descriptions Organisation

Table 3.6b Table 3.6b Collaborative Learning: Frequency of group work observed

Table $3.7 a$ Personalised Learning

Table 3.9a Table 3.9a Dialogic teaching and learning: comprehension and analysis activities

Table 3.10a Table 3.10a Assessment for learning (AfL) 


\section{Executive Summary}

The Effective Provision of Pre-School, Primary and Secondary Education (EPPSE 3-16) project is a large scale, longitudinal, mixed-method research study that has followed the progress of $3000+$ children since 1997 from the age of 3 to $16+$ years.

A continuing question for EPPSE was whether pre- and primary school experiences or children's early home learning environment (HLE) could reduce inequality. While the original studies found that parents' socio-economic status (SES) and qualifications were significantly related to child outcomes, they also found that the quality of the early HLE was important (Melhuish et al., 2008; Sammons et al, 2004). Also important, and particularly relevant to this study, was the extent to which educational influences (pre-school and primary school quality and effectiveness) also shaped children's educational outcomes. These reports were published as 'Variations in Teacher and Pupil Behaviours in Year 5 Classrooms' (Sammons et al, 2006) and 'The Influences of Teaching Quality on Children's Progress in Primary School' (Sammons et al., 2008).

This earlier research found that overall teaching quality is a significant predictor of better cognitive progress for children, and specific aspects of classroom processes were found to predict both better cognitive progress and social/behavioural development. For example, higher levels of disorganisation were related to poorer progress in Reading and Mathematics and increased hyperactivity and quality of pedagogy showed a strong relationship with children's progress in Mathematics (Sammons et al., 2008).

Since educational influences are mainly exerted through teaching, this suggested that an indepth evaluation of the pedagogical strategies used in the schools involved would be of both policy and practitioner interest. This study provides greater insights into effective primary pedagogical strategies in English and Maths.

Pedagogy is a contentious term (see Ko \& Sammons, 2010). Our definition is: Instructional techniques and strategies which enable learning to take place. It refers to the interactive process between teacher/practitioner and learner, and it is also applied to include the provision of some aspects of the learning environment (including the concrete learning environment, and the actions of the family and community). (Siraj-Blatchford et al., 2002)

Effectiveness is another controversial term. In educational effectiveness research the focus is on the teacher or school's contribution to pupil progress (Teddlie and Reynolds, 2000) Melhuish et al (2006:4) argued that:

Primary schools where children make significantly greater progress than predicted on the basis of prior attainment and intake characteristics can be viewed as more effective (positive outliers in value added terms). Primary schools where children make less progress than predicted can be viewed as less effective (negative outliers in value added terms).

This study explores the associations between value added measures of school effectiveness and variations in pedagogy. 


\section{Methodology}

During the primary phase (EPPE 3-11) of the longitudinal study the research team conducted contextualised, value-added analyses for all primary schools in England across three years $(2002-2004)$ from Key Stage 1 to Key Stage 2 (Melhuish et al, 2006). These analyses, based on multi-level modelling, considered children's progress and attainment while controlling for a range of background factors (e.g. gender).

As part of this, 125 primary schools, drawn from the 850 schools in the study, were selected for further study. The schools identified had a range of academically effective outcomes, including those with high, medium and low effectiveness scores, covered a range of contexts (inner city, shire and rural) and had at least four EPPE children on roll. Standardised assessments were used to measure the children's cognitive attainment in Reading and Mathematics and teachers completed profiles on their children's social/behavioural development. During field visits during the Spring and Summer terms of 2004 and 2005, researchers collected information about classroom practices and processes through classroom observations. They used two observational instruments: the Classroom Observation System for Fifth Grade (COS-5, NICHD, 2001) and the Instructional Environment Observation Scale (IEO, Stipek, 1999). Of the original sample, 82 primary schools with full data sets were included in this analysis.

Strict criteria were applied to identify three distinct groups of 'ideal type' schools: academically effective schools with good quality pedagogy (10 excellent schools), schools with medium academic effectiveness and medium quality pedagogy ( 9 good schools) and those with low academic effectiveness and poor quality pedagogy (10 poor schools). The remaining schools fell in-between/around these categories (apart from a small number [8 schools] that did not fit clearly into any category and were excluded). Each 'ideal type' group contained schools from a variety of settings (inner city, shire and rural) and had a range of levels of advantage of pupil intake (i.e. schools with higher and lower percentages of pupils eligible for free school meals [FSM]).

In order to develop the analytical framework of pedagogical strategies, professional focus group discussions, with a professor of education, the head teacher of a large primary school and a teacher were combined with a literature search to identify factors that contribute to effective classroom practice. An initial 40 strategies/factors (many with similar features) were collapsed into 11 main strategies.

\section{Findings for each of the Pedagogical Strategies}

\section{Organisation}

Teachers in excellent and good schools were rated highly on their organisational skills and wasted no time. The classroom routines were efficient and smooth. Children were responsible for their own time and resources: they knew what to do and they did it.

Teachers in excellent schools were rated exceptionally highly. Their resources were prepared ahead of time, well-managed during lessons, were particularly fit-for-purpose and tailored to the individual needs of their pupils. They made productive use of instructional time by maintaining good pace and by ensuring that every second of their lessons counted. Pupils in these classes had the highest ratings of self-reliance.

Year 5 classrooms in poor schools were rated significantly lower than the other groups on how well the teacher's resources were organised and how fit for purpose they were, how 
productively instructional time was used, the clarity of the teacher's expectations, including classroom routines, and the children's independence and self-reliance. Lessons were slow to start, pace was not maintained and time was wasted during transitions. Pupils in these classes received the lowest ratings of self-reliance.

\section{Shared objectives}

Teachers in excellent and good schools ensured that the concepts and ideas presented in lessons were understood by all children. They checked that children understood the main ideas of the lesson, intervened when understanding was not clear or complete and did this even when it meant changing the lesson or activity part way through.

Although most teachers were good at making sure the learning intentions of each lesson and activity were clear to the children (for example, by writing lesson objectives on the board), teachers in excellent schools were especially good at this. Pupils in these classes were very clear about what they were expected to achieve and how much time they had to do it in.

In contrast, objectives and learning concepts and ideas were less clear in poor schools. Teachers were slow to check, and to correct where necessary, their pupils' understanding of key concepts and ideas. Although children in these classrooms were aware of their lesson objectives, it was not clear whether or not they fully understood them or how to achieve them and there was much less focus and drive to meet these goals.

\section{Homework}

Although the use of homework was not systematically recorded when classes were observed, the researchers captured some data on this area. Teachers in excellent and good schools appeared to set homework that was more meaningful and more directly linked to what the children were learning. They had a more flexible approach to setting work to be completed outside of class time and this was sometimes in addition to the timetabled requirements. For example, they were more likely to make use of spontaneous learning opportunities that arose within lessons (e.g. asking the children to write the next paragraph of a story being written collaboratively by the whole class) that would allow children to extend and deepen their understanding of classroom learning.

In poor schools, teachers set homework simply because they were required to set it and the work itself did not appear to be expressly linked to what the children were learning in class. There were no examples of teachers using opportunities that arose during a lesson to set different/more homework than what was already planned.

\section{Classroom climate}

Classroom climate, the overall feeling in the classroom characterised by teacher-pupil and pupil-pupil relationships, was rated highly in excellent and good schools. Classrooms in excellent schools were rated exceptionally highly on positive classroom climate. Children were well-liked and respected by their peers and ratings of negative classroom climate were, as expected, very low.

The overall classroom climate in poor schools was often rated as unpleasant. Teachers were more likely to display negativity (disapproval, reprimands, expression of teacher's dislike, etc) and children in poor schools were less sociable and less cooperative than those in other schools.

\section{Behaviour management}

The differences between the three groups were evident when considering the management of behaviour. Children in excellent and good schools were less disruptive and rarely needed 
to be disciplined. Where teachers did need to correct behaviour, they used humour or a quiet reminder.

Although levels of indiscipline were also generally low in poor schools, children in these schools were more disruptive and teachers disciplined them more frequently. Discipline was often public and sometimes involved threats, personal attacks, shaming or belittling children. Levels of chaos and teacher over-control (rigid approaches designed to meet teacher's rather than children's needs) were significantly higher in poor schools.

\section{Collaborative learning}

Children in excellent schools spent the most time overall in collaborative learning situations (although the differences in the amounts of time children in different groups of schools spent in collaborative learning settings were not significant).

\section{Personalised teaching and learning}

Teachers in excellent and good schools were more likely to personalise their pupils' learning experiences. They did this by being sensitive to the individual needs of the children in their classes and by providing learning materials that were rich and varied. They were rated as very low in teacher detachment (for example distancing themselves from their pupils by staying and working at their desks, not offering feedback, not noticing children's behaviour or needs) and high when providing social support for student learning in literacy.

Teachers in excellent schools were exceptionally sensitive to the needs of the children in their classes and provided outstanding learning materials specifically chosen and adapted for their pupils. The individual needs of the Year 5 children in these schools were met through their teachers' friendly approach, high expectations and appropriately challenging and differentiated tasks.

Teachers in poor schools provided teaching and learning resources that were less varied and engaging and less likely to be appropriately differentiated, were less sensitive to their pupils' individual needs and more detached from their learning experiences.

\section{Making Links Explicit}

On the whole there were few instances of teachers making extra and cross-curricular links explicit. Teachers in excellent schools were better able to and more consistent at making links to areas outside the specific lesson. Teachers in poor schools rarely connected their lessons and activities with other subjects or with areas outside the classroom or school.

\section{Dialogic Teaching and Learning}

The extent of dialogic teaching (Alexander 2011) showed few differences between the three groups, except in Numeracy where teachers in excellent schools received the highest ratings on using dialogic teaching and learning. Teachers in excellent and good schools were rated significantly higher on dialogic teaching (Alexander 2011) for their use of analysis in maths and in the depth of their pupils' knowledge and understanding. They were also rated more highly on maths discussion and communication and on sharing the locus of Maths authority (Alexander 2011). In Literacy, they were rated higher on instructional conversations.

Children in poor schools spent less time learning and carrying out analysis, their teachers were less likely to encourage discussion, analysis and depth of understanding of mathematical concepts or to share the responsibility for learning with the children or to support and promote discussion for deeper understanding $\mathrm{n}$ literacy. 


\section{Assessment for Learning (AfL)}

Teachers in excellent and good schools provided more evaluative feedback than those in poor schools. In addition, teachers in excellent schools provided greater opportunities for their pupils to reflect on their learning through review than teachers in both good and poor schools. Teachers in good and poor schools did not differ in the extent to which they provided opportunities for pupils to reflect on their learning through review.

\section{Plenary}

Although data on the use of plenaries had not been collected for all schools in the sample, teachers in excellent and good schools were found to have included plenaries in their lessons almost twice as often as those in poor schools. In addition, those in excellent schools were more likely to use the plenary to provide opportunities for further discussion, to explore issues in more depth and to extend work and concepts covered in the lesson. This finding extends that reported by Sammons et al, (2006) which revealed a statistically significant link between use of the plenary and independent measures of observed quality based on both the COS5 and IOE instruments.

In poor schools, a plenary session was often not included at the end of the lesson and when it was, was most likely to be an opportunity for children to check their answers rather than an opportunity to deepen understanding.

\section{What differentiates best practice from good practice?}

Year 5 teachers in excellent schools (defined as those which are academically effective with good quality pedagogy):

- Have excellent organisational skills. They share clear learning objectives with the children in their classes and ensure that all pupils understand these objectives and their associated concepts. Their resources are extremely well organised and fit for purpose and their classroom routines are well-established, smooth and followed by all. Children in these classrooms know what they have to do, what to do if they need help and have more responsibility for managing their time and resources.

- Establish a positive classroom climate. In these classrooms, relationships between children and between adults and children are characterised by a true sense of liking and mutual respect and are often described as happy places with a "buzz" of productive learning activity. Children in these classrooms are less disruptive, behaviour management is handled sensitively and often through expectation and teachers rarely have to discipline children. Teacher sensitivity is high and teacher detachment low.

- Personalise their teaching. These teachers are sensitive to the needs and interests of their pupils and provide a variety of resources to suit the different needs of the individual children in their classes. Learning objectives are clear and shared and these teachers are more likely to make explicit the links between the learning and activities in the classroom and other subjects and the world outside the classroom. These teachers link their homework directly to what children are learning in their lessons and are more likely to take advantage of opportunities that arise during lessons to suggest learning activities that can take place out of class time.

- Use dialogic teaching and learning, especially for Numeracy. Children in their classrooms are more likely to work collaboratively, to take part in instructional conversations in Literacy, to have opportunities to receive evaluative feedback (from the teacher or from their peers) and they spend more time learning and performing analysis. 
In Maths, these teachers outperformed other in their use of Maths analysis, the depth of their pupils' knowledge and understanding, maths discourse and communication and their willingness to allow the children to also be the maths 'authority' in the classroom. The dialogue in these classrooms was genuinely two-way; teachers were open to pupils' suggestions and corrections and used these in their teaching.

- Made more frequent and better use of the plenary. Not only were these teachers about twice as likely to use a plenary in their lessons, they used the plenary to allow further discussion, exploration and extension, to provide opportunities for useful feedback and to consolidate and deepen understanding.

It is highly likely that good organisational skills, a positive classroom climate, personalised and highly interactive approaches to teaching and learning, dialogic teaching and learning and the use of a plenary session all interact with and reinforce each other. For example, dialogic teaching and learning would be impossible in settings with a negative classroom climate. Personalising children's learning requires good organisational skills and helps both to create a positive classroom climate and to encourage discussion. 


\section{Section 1 Introduction to the Effective Primary Pedagogical Strategies in English and Maths (EPPSEM) Study}

Around the start of the millennium, a photo of a child with a flame of inspiration in his eye was used as part of a teacher recruitment campaign. The look in the boy's eyes - the pennydropping moment - was meant to entice new recruits into teaching with the promise of an exciting and intrinsically rewarding career spent inspiring young people. Whether or not there are any official statistics on the effectiveness of that photo on teacher recruitment, it was a striking image that was intended to express what, for many, is the whole point of joining the teaching profession.

Experienced teachers, however, know that it's not just the creation of that spark, but finding a way to keep the flame burning, that sets apart good from excellent teachers. Learning and teaching are about turning that initial breakthrough into a long lasting foundation that will link to and support further learning. This, of course, is the multi-billion pound question: How can we create the ideal set of circumstances for teaching and learning that leads to inspiration, long-lasting learning, deep understanding and high achievement?

Even though the answer to the 'big' question is beyond the scope of this study (and there are probably a number of answers that vary with age, context and other factors), the present study offers a unique opportunity to look closely at what happens in some of the Year 5 classrooms of highly and moderately (excellent and good) academically effective primary schools as well as what happens (or doesn't happen) in some of the classrooms of schools with low academic effectiveness and achievement (poor schools).

\subsection{Introduction to the Effective Provision of Pre-School, Primary and Secondary Education (EPPSE 3-16) Research Project (1997 - 2013)}

The Effective Provision of Pre-School, Primary and Secondary Education (EPPSE 3-16) research project is a large scale, longitudinal, mixed-method research study (see Sammons et al 2005; Siraj-Blatchford et al 2006) that has followed the progress of over 3000 children from the ages of 3 to 16 years (Sylva et al 2010). A continuing question for EPPSE was whether pre and primary schools and children's home learning experiences could reduce inequality. While the original studies found that parents' socioeconomic status (SES) and qualifications were significantly related to child outcomes, it also found that the quality of the early Home Learning Environment (HLE) was important (Melhuish et al 2001; Sammons et al $2002 ; 2003 ; 2004)$. Particularly relevant to the present study, the research showed that educational influences (pre-school and primary school quality and academic effectiveness and a study of their impact on pupils' progress and development) also shaped children's educational outcomes (Sammons et al 2008a). Since educational influences are mainly exerted through teaching, this suggested that an in-depth evaluation of pedagogical strategies used in the Year 5 classrooms of more and less academically effective schools would be of both policy and practice interest. A sub-study of the EPPSE project was conducted and produced two reports: Variations in Teacher and Pupil Behaviours in Year 5 Classrooms (Sammons et al., 2006a) and The Influence of School and Teaching Quality on 
Children's Progress in Primary School (See Appendix 1 and Sammons et al., 2008). The EPPSEM study builds on these initial findings to provide greater insights into effective primary pedagogical strategies in English and Maths.

Section 1 of this report describes the background and aims of the EPPSEM study, provides working definitions for pedagogy and primary school academic effectiveness, considers some of the findings from the literature and outlines the research questions. Section 2 includes information on methodology, sampling procedures, the observational instruments and data collection preparation and analyses. Section 3 describes the main findings from these analyses. Section 4 offers some interpretations and potential implications of the findings, synthesises the results into an overall picture and addresses the EPPSEM research questions.

\subsection{Background and aim of the EPPSEM Study}

During the primary phase (EPPE 3-11, 2003 - 2008)) of the longitudinal study, the research team conducted contextualised, value-added analyses of pupil progress, based on national assessment results, for all primary schools in England across three years (2002 - 2004) from Key Stage 1 to Key Stage 2 (Melhuish et al 2006). These analyses, based on multilevel modelling, considered children's progress and attainment while controlling for prior attainment, gender, age, ethnic background, level of disadvantage, English as an Additional Language (EAL) and special educational needs (SEN).

The researchers found that while many of the above factors influenced children's progress and attainment, the greatest contributor to children's Key Stage 2 assessment results was their prior attainment. Nonetheless, there were significant differences in progress linked to the primary school attended. The findings suggested that a school's overall academic effectiveness might be closely linked to the schools' strategy for helping lower-achieving children make good progress.

As part of the primary phase of the research, Year 5 classrooms in 125 focal primary schools, drawn from the 850 schools attended by the EPPE 3-11 children, were selected for further study. The schools identified included those with high, medium and low academic effectiveness scores, covered a range of contexts (inner city, shire and rural) and had at least four EPPE children on roll (though not all of these children were in Year 5 or in the Year 5 classroom(s) being observed). The study was conducted in Year 5 to avoid possible influence from the national assessment focus in Year 6.

During this phase, the EPPE children's cognitive attainment in Reading and Mathematics was measured by independent, standardised assessments (NFER) and their teachers returned profiles on their social/behavioural development. During field visits, researchers collected information about classroom practices and processes through classroom observations. The researchers visited the Year 5 classes to investigate variation in observed practice. Later, their observational data was linked to other information to explore the relationships between pupil progress and classroom/school factors. The research also explored the associations between children's outcomes and several measures of school attributes as reported by inspectors from the Office for Standards in Education (Ofsted) such 
as overall school effectiveness, improvement and features of their learning and teaching. The findings are reported in three technical papers (Sammons et al., 2006a; 2006b; 2008). These reports identify the following key findings:

- there was significant variation in teachers' practice and children's responses in Year 5 classes was identified;

- overall, levels of pupil engagement were relatively high, classroom climate was generally positive and teacher "detachment" fairly low;

- pupil behaviour tended to be worse in schools where there were relatively more children eligible for free school meals (FSM), and also where the observed classroom climate was less favourable;

- more whole class than individual work occurred and collaborative and other types of group work were relatively uncommon;

- the quality of classroom practice was associated with the use of plenary sessions in literacy and numeracy lessons. Practice was found to be better in classes that used plenaries in both these subjects and poorer in classes where no plenary was observed in either. Plenaries, however, were only present in approximately half the Literacy or Numeracy lessons observed;

- the quality of Year 5 pedagogy and organisation and pupil behaviour was poorer in schools with higher levels of social disadvantage in their pupil intakes, and

- the quality of practice observed was better in schools that had been rated more highly in terms of overall school leadership, effectiveness and improvement on the last Ofsted inspection.

The following key messages are especially relevant to the equity agenda generally and for schools in more challenging circumstances:

- observed scores were lower for basic skills development, depth of subject knowledge, social support for learning in Mathematics, pupil engagement and classroom routines in Literacy, in classes with more disadvantaged intakes;

- scores for pedagogy in Literacy and subject development for Numeracy tended to be lower in schools with high levels of pupils eligible for free school meals (FSM), and

- the quality of teaching tended to be poorer in schools with higher levels of disadvantage, while the behavioural challenges in terms of pupil behaviour in class tended to be greater.

The research revealed statistically significant relationships between teachers' observed practice and children's academic and social/behavioural development. These are fully summarised in Appendix 1.

The full quantitative analyses enabled EPPE 3-11 to show how variations in Year 5 teachers' classroom practices predicted child outcomes in reading and mathematics and in social 
behaviour development. The key findings identified a number of important associations of interest to both policy makers and practitioners. However, the analytical strategy was predicated on quantitative measures of teaching, pedagogy and behaviours collected from numeric rating scales. This was sufficient to answer the main research questions at the time and the analyses were conducted within the resources allocated to the research. The classroom observational data, however, were more nuanced as the field researchers were asked to collect not only their global numeric ratings but also to take detailed qualitative field notes on what teachers did and how pupils responded. These notes were used to justify their quantitative ratings on the scales. Measures of quality of pedagogy that were taken using two instruments: the Classroom Observation System for Fifth Grade (COS-5, NICHD 2001, see Appendix 2) and the Instructional Environment Observation Scale (IEO, Stipek 1999, see Appendix 2). In compiling their notes, the researchers were also able to add fine grained or "thicker" descriptions of the pedagogy in Year 5 classrooms. Further analyses of the field notes provides greater understanding of the relationships between teaching and learning in Year 5 classes and better guidance concerning excellent, good and poor practice for both practitioners and policy makers..

The EPPSEM study extends the work previously described in the EPPE 3-11 analyses of Year 5 classrooms by looking in greater depth at the rich, qualitative descriptions of classroom environments, practices and interactions provided in the researchers' detailed notes of their classroom observations. We have full qualitative and quantitative data, including both sets of structured observations, for 82 Year 5 classes, and the final analysis has been based on these 82 primary schools that vary in academic effectiveness and observed pedagogical quality (based on the earlier quantitative analyses). The study has included a good selection of schools with more and less advantaged pupil intakes (as measured by the percentage of pupils eligible for FSM) in both the effective and ineffective schools. The aim of this report is to identify, within the descriptions, the pedagogical strategies used in these schools to consider whether teachers' strategies vary according to the level of effectiveness of the school and to illustrate these differences with examples from the actual observations. This allows us to add explanatory power to some of our statistical patterns in terms of child outcomes and observed pedagogical quality ratings.

\subsection{Some working definitions}

\subsubsection{Pedagogy}

Pedagogy is a contentious term (see Ko \& Sammons 2010). There are numerous definitions and much time has been devoted to debating their subtleties. Watkins and Mortimore (1999:3) defined pedagogy as "any conscious activity by one person designed to enhance the learning of another." Alexander $(2001 ; 2008)$ argued that pedagogy has been defined too narrowly in the past and he specifically criticised Watkins and Mortimore (1999) for a definition limited to the "actions" of teachers. This is a perspective that Alexander goes on to suggest is both anti-intellectual and instrumental in nature. These arguments may seem surprising as Watkins and Mortimore's acute awareness of the importance of context was obvious and they plainly shared many of Alexander's expressed concerns about prescriptive approaches to pedagogy. In fact Ireson, Mortimore and Hallam (1999:222) demonstrated a particularly clear recognition of the issues: 
If there are to be radical changes in pedagogy to equip young people for the future, then teachers and lecturers must have an understanding of learning processes and the factors that affect them. They must also be learners too . . . for example, if teachers are to enable learners to develop a better awareness of their own learning, they too will need to develop awareness of their own learning, along with pedagogic strategies to encourage it.

Ireson, Mortimore and Hallam (ibid) went on to argue that:

The theoretical basis of new pedagogical initiatives needs to be established, researched and debated if teachers are to take on the role of the architects rather than the bricklayers of learning.

The role of teachers as architects implies a broad and carefully considered set of approaches designed to meet a wide range of contexts and individual needs.

Alexander (2008) described a holistic, socio-cultural analytic framework that many studies have now sought to apply in studying the mediating influences that play a part in the development of policy and practice. It is important to recognise, however, that this is not the only legitimate and useful analytic framework to adopt in the study of pedagogy. While holistic analysis may be considered to provide a necessary and important critical tool for engagement with national policy, it is arguably a much more focused approach that is required to support the day-to-day development of learning and teaching for practicing professionals.

Gage (1985) defined pedagogy as 'the science of the art of teaching'. This is a science that is continually developed by innovative teachers and the academic researchers who study their practice. It is in this way that many pedagogic strategies such as assessment for learning (AfL) (Black and Wiliam 1998) and dialogic enquiry (Wells 1999) have become accepted as established (and practiced) knowledge. Effective teachers must have an awareness of this body of knowledge, but they must also be considered to be practising artists in selecting the most appropriate strategies to apply in any given learning and teaching context. The best teachers creatively draw upon their knowledge of the interests and capabilities of the children in their care, and also upon a wide range of material, cultural and intellectual resources to provide the children with the most effective, engaging, memorable and rewarding learning experiences possible on a day-to-day basis. And, just as a kind of scientific 'development' may be seen in the work of many great painters, the performance of effective teachers also develops as they continually reflect upon, hypothesise about, critically evaluate and moderate their practice to achieve excellence. So much is predicated on the teacher's 'contextual literacy' of the needs and backgrounds of their students.

Joining the debate is beyond the scope of this study so it was important to establish a working definition to frame our work. The perspective EPPSEM adopts on pedagogy is also evidence-based and resonates with a wide range of empirical findings. We have adopted the definition used on previous EPPE research work that states that pedagogy refers to: 
the instructional techniques and strategies which enable learning to take place. It refers to the interactive process between teacher/practitioner and learner, and it is also applied to include the provision of some aspects of the learning environment (including the concrete learning environment, and the actions of the family and community). (Siraj-Blatchford et al 2002:p10)

\subsubsection{Effectiveness}

Effectiveness, whether at school, classroom or teacher level, is another controversial term. Ko and Sammons (2010:p45) noted that "effectiveness" is both a political and emotional term and so the way in which it is measured varies from time to time and context to context:

'Effectiveness' can be viewed as a politically contested term that can evoke strong emotions because of its perceived links with notions of professional competency and high stakes accountability in some systems and because it can be thought to question teachers' professional autonomy. Notions of what constitutes high quality or good teaching, the idea that teaching is an art rather than a science are sometimes used to question a concern with the narrower concept of effectiveness. However, notions of what constitutes 'good' or 'high' quality practice can vary markedly at different times and in different contexts.

Sammons \& Ko (2008) produced an analysis of observational data collected for the mixed methods Effective Classroom Practice project (Day et al, 2008). They noted that some theories of teacher effectiveness argue that this is a differentiated model (in other words, that teachers' practice and effectiveness may vary over time and in different contexts). They compare this to the generic model that suggests some teachers' practice may be more effective than others (i.e. one can distinguish between more effective and less effective teachers). Their data provided some evidence to support both concepts.

In this recent multi-disciplinary study into effective classroom practice, Day et al's (2008) study investigated the observed practice of teachers in schools identified as more effective using contextualised, value-added measures of academic performance. Comparisons were made between the observed practices of effective and the most effective teachers observed in the study:

Although many aspects of effective classroom practice were utilised by both effective and more effective teachers, the more effective teachers seemed to possess and combine a greater range of teaching and learning strategies . . . in consistently positive and more reflective, complex and contextuallyresponsive ways (Day et al, 2008).

These findings provided further support for both concepts of effectiveness. While the teaching and learning strategies that are considered to be the most effective depend on the context and change over time, the individual abilities of teachers to adapt to these changes also vary.

For the purposes of the EPPSEM study, a value-added measure of academic effectiveness that provided an indication of whether a school's pupils were performing at, below or above 
their expected level of academic achievement, based on national data, was the most useful because it simplified the grouping of schools and facilitated comparisons between them. Melhuish et al (2006:4) argued that:

Primary schools where children make significantly greater progress than predicted on the basis of prior attainment and intake characteristics can be views as more effective (positive outliers in value added terms). Primary schools where children make less progress than predicted can be viewed as less effective (negative outliers in value added terms)

and this is the approach we have adopted.

\subsection{Effective pedagogical strategies: what the literature tells us}

When reviewing the literature on academic effectiveness and quality of pedagogy, a number of themes emerge. Some of these themes (e.g. leadership, monitoring and use of assessment data, communication with parents) were not addressed in the research instruments used for this study and so are not included in this report. A brief review of the relevant literature for each of the strategies that has been included in this study is provided below.

Organisation (of classroom routines and the structure of lessons) is almost universally accepted as an important element of effective pedagogy. Previous EPPE research (Sammons et al., 2008) reported that children in disorganised Year 5 classrooms made less progress in Reading and Maths than their peers in better organised classrooms. Levels of hyperactivity were also increased in these classrooms. Regarding lesson structure, observed practice was rated more favourable in classes where plenaries - an essential part of each lesson as outlined in the National Strategies - took place (Sammons et al 2006a; 2006b).

The Cambridge Primary Review states that good teaching "is well-organised and planned" (Alexander, 2010:281). The Rose Review (2006) of the teaching of early reading pointed out that difficulties in learning to read can be caused by poor classroom organisation and that efficient classroom organisation and management, as well as clear lesson objectives, are characteristics of good and outstanding teaching. Pace is a good indicator of how well planned a lesson is and Sharples et al (C4EO 2011:14) included pace in the list of effective classroom interventions that help "close attainment gaps for children living in poverty". The need for careful and flexible planning as an effective pedagogical strategy for teaching Maths was highlighted by Williams (2008), particularly as a way of adapting lessons to the sometimes unpredictable needs of pupils. Most research indicates that effective organisation is a powerful strategy.

Lesson structure is considered important in the Primary National Strategy (DfEE 1999, DfES 2001). Numeracy lessons are divided into three parts: a whole class starter (10-15 min), main lesson (30-40 min beginning with whole class instruction and followed by individual or group work) and then a whole class structured plenary session (10-15 min). Literacy lessons are more tightly structured and are divided into four parts: text level work (15 min), sentence or word level work (15 min), group work (20 min) and a whole class plenary (10 
min). The emphasis placed on defining the structure of these lessons is based on a view that lesson structure is key to successful teaching (Muijs \& Reynolds 2011).

Sharing learning objectives and goals also feature in the literature. The Cambridge Primary Review stated that "learning is primarily a social activity which requires common goals and shared activities. This should be the teacher's starting point ..." (Alexander 2010:p289). Ensuring that all members of a class are clear on what those goals are, and that everyone is working towards them, are important components of teaching and learning. For children to successfully achieve their learning objectives, they must understand what those objectives are and how to achieve them.

Homework (school work set for children to complete outside of school hours) has been used to supplement the curriculum since the mid $19^{\text {th }}$ Century. Views on whether or not setting homework is an effective pedagogical strategy have changed, however, "depending on political, economic, social and educational factors" (Hallam 2004:1). Homework is sometimes seen as an essential part of a child's education and at other times, considered an infringement on children's freedom and play time.

Homework is used for many purposes: consolidating and extending learning that has taken place in the classroom, practising basic skills (e.g. times tables, spellings), assessment (of both the child and the teaching), developing independent study skills, time management (work completed at home can free space on the timetable for other activities), building homeschool links and meeting the requirements of the school, the parents, statutory or recommended policy and both internal and external inspectors (Hallam 2004; Ofsted, 1995). The Ofsted report (1995) on homework in primary and secondary schools found that "in general, teachers and parents believed that homework played a valuable part in pupils' education" (Ofsted 1995:4).

In most English schools today, homework is considered an integral part of a child's education. The DfEE recommends 30 minutes of homework per day for children in Years 5 and 6 (DfEE 1998). Most schools have homework policies and timetables that often prescribe the days that homework should be set as well as the amount of time children should spend on each subject. A typical example of the approach many schools take to homework is exemplified in a letter sent to parents of Year 7 and Year 8 children at a West Midlands school:

Dear Parent, I am sure that we all believe that homework/independent study is an essential part of each student's education. It:

- assists and improves learning

- enables teachers and students to cover more ground

- consolidates and reinforces skills and understanding gained in the classroom

- encourages students to develop independent learning and research skills as a parent it informs you about the school curriculum and provides you with the opportunity to assist your son/daughter with their time management and organisational skills (Connolly, 2008). 
The letter then suggests, in 20 or 30 minute blocks, the approximate amount of time students should devote to each subject per week. This communication from school to parents is a good illustration of the commonly held assumption that homework can provide a positive contribution to children's education.

Despite the current emphasis on setting homework, it is important to look at the evidence that might suggest that homework improves achievement. Compiling the evidence on this topic is complex. Sharp (2001) noted some of the difficulties in trying to establish the link between homework and achievement. Deciding how to measure homework is problematic because it can be measured as the total amount of time suggested by the teacher, as the amount of time children report they actually spend on it or by the frequency/regularity with which it is set. Children's accounts of the time they spend on homework may be inaccurate and teachers may be unaware of discrepancies between the time they allow for completion of the work and the actual time taken by their pupils.

Further complicating the issue is that while homework might be set, this does not guarantee that it has been completed, fully or partially, that teachers have checked that their pupils have completed the assigned work or that the homework has been assessed or that feedback, including an opportunity for children to reflect on the teacher's guidance, has been given. For example, Ofsted's (1995) review of homework in primary and secondary schools found that "in those schools where the process of setting and marking homework was an integral and regular part of the working week, standards were generally very satisfactory and in some cases good (Ofsted, 1995:5-6). The impact of homework on achievement, then, may vary according to whether it is measured by overall time (and this, in turn, may vary depending on whether time is set by the teacher or measured by the pupil), the frequency and the regularity with which homework is set and marked and the standard to which it has been completed. When considering research results on homework and achievement, these limitations need to be taken into account.

Hallam (2004) examined the evidence on homework and achievement. She found that results were variable. Some studies reported that homework had no effect on attainment, some indicated homework had a positive effect on attainment and still others found positive effects on attainment for older children only. When considering all the evidence, Hallam concluded that the relationship between homework and attainment was curvilinear: increasing the amount of homework is beneficial up to a certain point after which the gains associated with further homework are small. Hallam explained that this relationship makes sense because as with any skill, extensive practice gives large gains early on but as time goes on, the gains become smaller. She concluded, "Overall, most studies relating to attainment offer support for pupils doing homework but only at a moderate level." (Hallam, 2004:32). Sharp et al (2001) came to the same conclusion.

In an earlier study, Sharp (2000) found that evidence to support the link between homework and achievement during the primary school years was inconclusive, apart from its impact on lower achieving children if their parents were informed and involved. Using homework to help these children was only effective, however, when parents were positive and encouraging and where they modelled the effort and work ethic expected of the child. 
A concern raised by Alexander's (2010) review of the primary curriculum was that homework might actually increase the gap between the more and less advantaged. Children from impoverished homes are less likely to have parents able to support them in their homework, to have an appropriate space in which to do their work and to have access to extra resources (computers, books and library resources outside the school, etc). In contrast, children from better off homes are more likely to have parents able to monitor and support their homework, a good environment in which to work and access to a large number of additional resources. This could mean that children from affluent families could make significant gains from the additional learning opportunities provided by their homework. Alexander concluded, however, that as evidence of the influence of homework on achievement was tenuous at best, homework was unlikely to exacerbate the differences between the more and less advantaged. The EPPE research for pupils' progress from Year 1 to Year 5 indicated that in schools where teachers reported they gave more emphasis to homework, the EPPE children made significantly more progress (Sammons et al, 2008)

The classroom environment contributes to how well children learn. Although previous EPPE work (Sammons et al 2006a; 2006b) reported generally positive classroom climates and low levels of teacher detachment, there was considerable variation. This meant that some children were leaning in much less favourable environments.

One of the main contributors to classroom climate is the relationship between teachers and pupils. Whitebook et al (2009:p10) considered the body of research evidence and concluded that "certain types of teacher-child relationships are all associated with better developmental outcomes for children." Keiser and Schulte (2009) recommended that school climate should be objectively assessed because it can influence pupil outcomes. When considering the impact of student-teacher relationships on gifted students, Kesner (2005:p222) used Pianta's (1991) Student-Teacher Relationship Scale and found:

. . . a highly successful teacher of the gifted is able to inspire and motivate students, reduce tension and anxiety for gifted students and appreciate their high levels of sensitivity. A teacher must have a positive interpersonal relationship with his/her gifted students in order to accomplish these things. The results from this study indicate that this type of relationship is possible. These positive relationships with gifted students suggest that teachers were able to meet the affective needs of the gifted students in their classrooms.

Providing a safe, supportive environment for students is another important aspect of classroom climate. Murphy and Milner (2009:p90), in their study of 300 secondary students (Grades 9-12), noted that:

All four teachers worked to craft a safe and comfortable learning environment. This appeared to factor in to student engagement in class. Teachers encouraged students to be supportive of one another and did not tolerate negative remarks directed from student to student. This would seem to also increase student confidence as well as the frequency of student comments and questions. 
Children need an environment that encourages them to safely explore and try new things without fear of ridicule or unwarranted reprimand.

The Cambridge Review states that good teaching "depends on effective classroom management of behaviour" (Alexander, 2010, p281). Children cannot learn effectively in an environment where other pupils persistently misbehave and disrupt lessons. Although the original EPPE study found that "observed 'off-task' behaviour is lower than that found in studies in the 1970s and 1980s" (Sammons et al 2006a, p13), there was enough variation to suggest that behaviour management remains a significant problem in some schools and adversely impacts on the educational experiences of the children in those classrooms.

In their review of best evidence from international experimental research, Sharples et al (C4EO 2011:p14) found that:

Classroom interventions that close attainment gaps for children living in poverty adopt proven classroom management strategies (e.g. rapid pace of instruction, using all-pupil responses, developing a common language around discipline).

When they considered UK-based evidence on what works for schools in high poverty areas, they found that managing behaviour was an important factor in any school, and not just for those in areas of greater deprivation. Specifically:

- cultivating values of respect, good behaviour and caring - supported by a clear approach to discipline

- understanding and developing staff and pupils with personal and professional support

- having clear lines of authority, responsibility, accountability and autonomy

all contributed to improving children's outcomes (C4EO 2011:p21).

Comparable to the findings of Galton et al (1999), EPPE research indicated that children spent very little time working in truly collaborative settings. Although children are generally seated in groups in primary schools, both Galton et al (1999) and the EPPE research found that children spent most of their time in whole class settings with just over a third of their time spent working individually. Once again, however, there was some variation between classrooms and some children spent much more time working collaboratively in small and large groups than others.

Cooperative learning has been shown to significantly raise outcomes, especially for children living in poverty (C4EO 2011). For example, a number of programmes in the U.S. that used cooperative learning, including one used for teaching phonics, were found to be very effective (C4EO 2011).

The importance of personalised learning is discussed in the Cambridge Primary Review (Alexander 2010) which states that teaching needs to be adapted to the specific needs of the learner. Teachers need to understand children's developmental needs so that their teaching 
can be personalised by building on prior pupil knowledge, looking forward to the next steps and stimulating children's creativity and imagination.

Dialogic teaching and learning make essential contributions to children's learning. Language, and the importance of meaningful, interactive discussion, were highlighted by Wells (1999:pxi) who wrote that "education should be conducted as a dialogue about matters that are of interest and concern to the participants." The contribution of language on children's learning extends to every area of the curriculum, including Mathematics. Research investigating the link between language and Mathematics has shown that children with language difficulties also demonstrate difficulties in Mathematics that cannot be accounted for by other factors such as memory or processing speed (Cowan et al, 2005; Donlan et al, 2007). The importance of language, shared discussions and one person's input informing another's are all significant contributors to children's education.

Spoken language is raised as an important element of effective pedagogy in every review of pedagogical strategies and is, in fact, the only recommendation The Cambridge Primary Review makes about pedagogy. Alexander (2010:496) argues that teachers should make their own decisions about how to teach but specifically states (recommendation 61):

We do not nominate any 'best buys' from recent pedagogical fads and fashions. At the same time, we note the extent to which research from many different sources converges on language, and especially spoken language, as one of the keys to cognitive development, learning and successful teaching and indeed to the learner's later employment and democratic engagement. In many classrooms . . . talk remains far from achieving its true potential. We urge all concerned, especially teachers and researchers, to act together to effect the pedagogical transformation which is needed, and which in some schools and local authorities has already begun.

Black et al., (2003) explained that assessment for learning (AfL) is "usually informal, rooted in teaching and learning, and can occur many times in every lesson. 'Assessment', in the context of this study, refers to all the activities undertaken by teachers and their students which provide feedback that shapes and develops the teaching and learning activities engaged in by the class. This becomes 'formative assessment' when the evidence is used to adapt the teaching work to meet the students' learning needs". (http://www.gtce.org.uk/pdf/teachers/rft/afl_prac0904). When looking at the test results of 25 secondary school classes, Black et al., (2003) found that classes whose teachers had implemented assessment for learning had results that were $15 \%$ higher than classes not using AfL. In the Cambridge Primary Review, Alexander (2010:p290) supported the view that "assessment for learning helps teachers promote learning how to learn".

The plenary is an important component of the three part lesson format suggested by the National Strategies and has been found to play an important role in children's learning. Although not present in about half of all lessons observed, previous EPPE research involving systematic observations of Year 5 classes revealed (Sammons 2006a, p25): 
The quality of teaching and pupil response was found to be consistently higher in classes where a plenary was used in both literacy and numeracy lessons and lowest in classes where no plenary was used in either subject.

The plenary is intended to play an important role at the end of most lessons so that time for consolidation, reflection and extension is built into every lesson, that is, dialogic teaching.

\subsection{The research questions}

The purpose of the EPPSEM study was to analyse effective pedagogical strategies in English and Maths in Key Stage 2. The study's main aims were to identify:

- the specific teaching behaviours in Year 5 classrooms associated with more or less effective impact on student outcomes using the detailed observations of Year 5 Literacy and Numeracy classroom practices in 125 classes (93 schools, 82 with complete data sets) during the spring and summer terms of 2004 and 2005.

- which were the most and least effective lessons and then analyse the lesson plans, the interaction within lessons, teacher-pupil dialogue, and issues of (dis)organisation and behaviour.

\section{Section 2 Method}

\subsection{The sample}

Of the 125 focus schools identified in the initial EPPE 3-11 study, 82 schools had full data sets that included measures of primary school academic effectiveness (contextual valueadded residual scores based on national assessment data) and measures of quality of pedagogy that were taken using two instruments: the Classroom Observation System for Fifth Grade (COS-5 NICHD, 2001, see Appendix 2) and the Instructional Environment Observation Scale (IEO Stipek, 1999, see Appendix 2). These 82 schools were selected for further qualitative case study analysis. Within the sample, three distinct groups of 'ideal types' of 'typical' schools were identified: academically effective schools with good quality pedagogy (10 schools referred to as Group A or Excellent schools), schools with medium academic effectiveness and medium quality pedagogy ( 9 schools referred to as Group B or Good schools) and those with low academic effectiveness and poor observed quality of pedagogy (10 schools referred to as Group C or Poor schools). The remainder of the schools fell in between or around these categories, apart from a small number (8 schools) that did not fit clearly into any category and so were excluded. Each 'typical' effectiveness and quality of pedagogy group contained schools from a variety of settings (inner city, shire and rural) and had a range of levels of disadvantage/advantage of pupil intake (i.e. schools with higher and lower percentages of pupils eligible for free school meals [FSM]). A complete list of schools and their characteristics can be found in Appendix 3. 


\subsubsection{Sampling procedure}

i) Group A: Excellent schools. Academically effective schools with good quality pedagogy

In order to identify which pedagogical strategies being used in Year 5 classrooms were particularly effective, the analysis was carried out in stages. As the researchers mainly observed English and Maths lessons, it was important to choose Year 5 classrooms in schools with high academic effectiveness ratings on contextualised, value-added analyses of National Assessment outcomes in at least one of these subjects. To be included in this group, a school's English and Maths National Assessment residual ${ }^{1}$ scores had to be high $(\geq 4)$ in both subjects or high $(\geq 4)$ in one subject and medium $(=3)$ in the other.

More general measures of pedagogical quality in the classroom were also important and so ratings on both classroom observation scales (Instructional Environment Observation (IEO) and Classroom Observation System for Fifth Grade (COS-5)) were conducted (see below and Appendix 2 for an explanation of these instruments). Schools included in Group A, the 'academically effective, good quality pedagogy group', were rated as having at least medium (IEO=2; COS-5=3) pedagogical quality on the rating scales with one or both ratings in the category 'high quality' (IEO=3; COS-5 $\geq 4$ ). The only other schools included in Group A were those with medium ratings on both pedagogical quality scales where both the English and Maths residual scores were rated as high (RS $\geq 4)$. The criteria were stringent and only ten of the 82 schools (12\%) fell into the 'academically effective, good quality pedagogy' category.

Table 2.1a. Criteria for inclusion in Group A: Excellent schools (academically effective, good quality pedagogy) *

\begin{tabular}{|c|c|c|}
\hline Source & $\begin{array}{l}\text { Possible scores } \\
\text { range from } \\
\text { (low to high) }\end{array}$ & $\begin{array}{l}\text { Minimum scores required to be } \\
\text { included in the academically } \\
\text { effective, good quality pedagogy } \\
\text { group }\end{array}$ \\
\hline $\begin{array}{l}\text { National Assessment Residual } \\
\text { Scores (RS) (a measure of school } \\
\text { effectiveness) }\end{array}$ & 1 to 5 & $\begin{array}{l}R S \geq 3 \text { in both English and Maths } \\
\text { AND } \\
\text { at least one } R S \geq 4\end{array}$ \\
\hline $\begin{array}{l}\text { Instructional Environment } \\
\text { Observation (IEO) }\end{array}$ & 1 to 3 & $\begin{array}{l}I E O \geq 2 \text { if } \operatorname{COS}-5=5 \\
O R \\
I E O=3 \text { if } \operatorname{COS}-5=3 \text { or } 4^{\dagger}\end{array}$ \\
\hline $\begin{array}{l}\text { Classroom Observation } \\
\text { System (COS-5) }\end{array}$ & 1 to 5 & $\begin{array}{l}\text { COS }-5 \geq 3 \text { if IEO }=3 \\
\text { OR } \\
\text { COS }-5=5 \text { if IEO }=2^{\dagger}\end{array}$ \\
\hline
\end{tabular}

Percentage of pupils eligible for free school meals (FSM) was available for each school and was checked to ensure that schools with both higher and lower proportions of pupils eligible for FSM were included in each category.

\footnotetext{
${ }^{1}$ the residual score is a measure of school effectiveness after allowing for pupils' initial ability and school intake characteristics, see Melhuish et al., 2006
} 
** Schools where quality of pedagogy on both scales was medium (IOE=2; COS-5=3) but both residual scores were high $(R S \geq 4)$ would also have been included in this group however none of the schools fell into this category.

Table 2.1b Characteristics of the ten typical academically effective schools with good quality pedagogy (Group A: Excellent schools)

\begin{tabular}{|c|c|c|c|c|c|}
\hline School & $\begin{array}{l}\text { Instructional } \\
\text { Environment } \\
\text { Observation } \\
\text { (IEO) }\end{array}$ & $\begin{array}{c}\text { Classroom } \\
\text { Observation } \\
\text { System for Fifth } \\
\text { Grade (COS-5) }\end{array}$ & $\begin{array}{r}\text { National } \\
\text { Contextu } \\
\text { Added (Res } \\
\text { Maths }\end{array}$ & $\begin{array}{l}\text { ssessment } \\
\text { ised Value } \\
\text { dual) Scores } \\
\text { English }\end{array}$ & $\begin{array}{c}\text { Level of } \\
\text { Disadvantage of } \\
\text { Intake (\% of Pupils } \\
\text { Eligible for FSM) }\end{array}$ \\
\hline S01 & 3 & 5 & 3 & 4 & 0 \\
\hline S02 & 3 & 3 & 5 & 4 & 22 \\
\hline S03 & 2 & 5 & 4 & 4 & 32 \\
\hline S04 & 3 & 4 & 3 & 4 & 31 \\
\hline S05 & 3 & 4 & 3 & 5 & 16 \\
\hline S06 & 3 & 3 & 5 & 3 & 3 \\
\hline S07 & 3 & 4 & 3 & 4 & 18 \\
\hline S08 & 3 & 4 & 4 & 4 & 12 \\
\hline $\mathrm{S} 12$ & 3 & 3 & 3 & 4 & 31 \\
\hline S13 & 3 & 4 & 4 & 3 & 49 \\
\hline
\end{tabular}

For the second part of the analysis, two further 'ideal types' of 'typical' groups of schools were identified: schools with medium academic effectiveness and medium observed quality of pedagogy (Group B: Good schools) and schools with low academic effectiveness and poor quality of observed pedagogy (Group C: Poor schools). The aim was to identify schools that were typical of these groups and then analyse, compare and contrast the pedagogical strategies used within all three groups of 'typical' Year 5 classrooms. This would provide a clearer picture of what was happening at the two extremes of the continuum as well as what was happening in the typically 'good or average' classrooms (Group B, medium academic effectiveness, medium quality pedagogy). Although the aim was not to necessarily have exactly equal numbers of schools in each group, by applying the criteria of each group stringently, the groups were, in fact, almost equal in size. As well as the ten excellent schools, there were nine schools good schools in Group B (described below) and ten schools poor schools in Group C (also described below).

ii) Group B: Good schools. Schools with medium academic effectiveness and medium quality pedagogy

These schools are the ones with 'average' results (i.e. scores at the median values) on all measures and once again, the criteria applied to identify typical schools were stringent. To be included in this group, a school's English and Maths National Assessment residual scores had to be medium $(=3)$ and ratings on both classroom observation scales (Instructional Environment Observation (IEO) and Classroom Observation System (COS-5) also had to be medium (IEO=2 and COS-5=3). Nine schools fell into this category. 
Table 2.1c. Criteria for inclusion in Group B: Good schools (medium academic effectiveness and medium quality pedagogy)*

\begin{tabular}{|lcl|}
\hline Source & $\begin{array}{c}\text { Possible scores } \\
\text { range from } \\
\text { (low to high) }\end{array}$ & $\begin{array}{l}\text { Minimum scores required to be } \\
\text { included in the medium academic } \\
\text { effectiveness medium quality } \\
\text { pedagogy group }\end{array}$ \\
\hline $\begin{array}{l}\text { National Assessment Residual } \\
\text { Scores (RS) (a measure of school } \\
\text { effectiveness) }\end{array}$ & 1 to 5 & $\mathrm{RS}=3$ in both English and Maths \\
$\begin{array}{l}\text { Instructional Environment } \\
\text { Observation (IEO) }\end{array}$ & 1 to 3 & IEO $=2$ \\
$\begin{array}{l}\text { Classroom Observation } \\
\text { System (COS-5) }\end{array}$ & 1 to 5 & COS-5 $=3$ \\
\hline
\end{tabular}

** Percentage of pupils eligible for free school meals (FSM) was available for each school and was checked to ensure that schools with both higher and lower proportions of pupils eligible for FSM were included in each category.

Table 2.1d Characteristics of the nine typical schools with medium academic effectiveness and medium quality pedagogy (Group B: Good schools)

\begin{tabular}{|c|c|c|c|c|c|}
\hline School & $\begin{array}{c}\text { Instructional } \\
\text { Environment } \\
\text { Observation } \\
\text { (IEO) }\end{array}$ & $\begin{array}{c}\text { Classroom } \\
\text { Observation } \\
\text { System for } \\
\text { Fifth Grade } \\
\text { (COS-5) }\end{array}$ & $\begin{array}{c}\text { National Assessment } \\
\text { Contextualised Value Added } \\
\text { (Residual) Scores } \\
\text { Maths }\end{array}$ & $\begin{array}{c}\text { English } \\
\text { Eisadvantage of } \\
\text { Intake (\% of } \\
\text { Pupils Eligible for } \\
\text { FSM) }\end{array}$ \\
\hline S25 & 2 & 3 & 3 & 3 & 35 \\
\hline S26 & 2 & 3 & 3 & 3 & 7 \\
\hline S27 & 2 & 3 & 3 & 3 & 11 \\
\hline S28 & 2 & 3 & 3 & 3 & 5 \\
\hline S29 & 2 & 3 & 3 & 3 & 3 \\
\hline S30 & 2 & 3 & 3 & 3 & 7 \\
\hline S31 & 2 & 3 & 3 & 3 & 19 \\
\hline S32 & 2 & 3 & 3 & 3 & 42 \\
\hline S36 & 2 & 3 & 3 & 3 & 14 \\
\hline
\end{tabular}

iii) Group C: Poor schools. Schools with low academic effectiveness and poor quality pedagogy

Schools with the lowest combination of academic effectiveness and quality of pedagogy formed Poor schools, Group C. To be included in this group, a school's English and Maths National Assessment residual scores had to be low $(\leq 2)$ in both subjects or low $(\leq 2)$ in one subject and medium $(=3)$ in the other. Schools included in the 'low academic effectiveness, poor quality pedagogy' group were rated as having at best medium pedagogical quality on the rating scales (IEO=2; COS-5=3) with at least one of the ratings in the category of "poor quality" (IEO=1; COS-5 $\leq 2)$. The only other schools included in Group C were those with 
medium ratings on both pedagogical quality scales where both the English and Maths residual scores were rated as low (RS $\leq 2)$. Once again, the criteria were stringent and only ten of the 82 schools fell into the "low academic effectiveness, poor quality pedagogy" category.

Table 2.1e Criteria for inclusion in Group C: Poor schools (low academic effectiveness, poor quality pedagogy)*

\begin{tabular}{|c|c|c|}
\hline Source & $\begin{array}{l}\text { Possible scores } \\
\text { range from } \\
\text { (low to high) }\end{array}$ & $\begin{array}{l}\text { Minimum scores required to be } \\
\text { included in the low academic } \\
\text { effectiveness, poor quality } \\
\text { pedagogy group }\end{array}$ \\
\hline $\begin{array}{l}\text { National Assessment Residual } \\
\text { Scores (RS) (a measure of school } \\
\text { effectiveness) }\end{array}$ & 1 to 5 & $\begin{array}{l}\text { RS } \leq 3 \text { in both English and } \\
\text { Maths } \\
\text { AND } \\
\text { at least one RS } \leq 2\end{array}$ \\
\hline $\begin{array}{l}\text { Instructional Environment } \\
\text { Observation (IEO) }\end{array}$ & 1 to 3 & $\begin{array}{l}\mathrm{IEO} \leq 2 \text { if } \mathrm{COS}-5=1 \\
O R \\
\mathrm{IEO}=1 \text { if } \operatorname{COS}-5=2 \text { or } 3^{\dagger}\end{array}$ \\
\hline $\begin{array}{l}\text { Classroom Observation } \\
\text { System (COS-5) }\end{array}$ & 1 to 5 & $\begin{array}{l}\operatorname{COS}-5 \leq 3 \text { if the IEO }=1 \\
\operatorname{COS}-5=1 \text { if the IEO }=2^{\dagger}\end{array}$ \\
\hline
\end{tabular}

* $\quad$ Percentage of pupils eligible for free school meals (FSM) was available for each school and was checked to ensure that schools with both higher and lower proportions of pupils eligible for FSM were included in each category.

** Schools where quality of pedagogy on both scales was medium (IOE=2; COS-5=3) but both residual scores were low $(\mathrm{RS} \leq 2)$ were included in this group. Three schools fell into this category.

Table 2.1f Characteristics of the ten typical schools with low academic effectiveness and poor quality pedagogy (Group C: Poor schools)

\begin{tabular}{|c|c|c|c|c|c|}
\hline School & $\begin{array}{c}\text { Instructional } \\
\text { Environment } \\
\text { Observation } \\
\text { (IEO) }\end{array}$ & $\begin{array}{c}\text { Classroom } \\
\text { Observation } \\
\text { System for Fifth } \\
\text { Grade (COS-5) }\end{array}$ & $\begin{array}{c}\text { National Assessment } \\
\text { Contextualised Value } \\
\text { Added (Residual) Scores } \\
\text { Maths } \\
\text { English }\end{array}$ & $\begin{array}{c}\text { Level of } \\
\text { Disadvantage of } \\
\text { Intake (\% of } \\
\text { Pupils Eligible } \\
\text { for FSM) }\end{array}$ \\
\hline S73 & 1 & 3 & 2 & 3 & 8 \\
\hline S74 & 2 & 3 & 2 & 2 & 10 \\
\hline S75 & 2 & 3 & 2 & 2 & 39 \\
\hline S76 & 1 & 1 & 2 & 2 & 53 \\
\hline S77 & 2 & 1 & 3 & 2 & 12 \\
\hline S78 & 2 & 1 & 2 & 2 & 13 \\
\hline S79 & 2 & 3 & 2 & 3 & 40 \\
\hline S80 & 1 & 2 & 3 & 2 & 15 \\
\hline S81 & 1 & 1 & & & 16 \\
\hline S82 & 1 & 1 & 2 & 2 & \\
\hline
\end{tabular}


For the final part of the analysis, once 'typical' schools had been identified, the remaining schools were grouped more loosely into categories that occasionally overlapped: schools that fell between each of the extremes and the 'average (Group B) and those that didn't quite meet the criteria of the 'average' school but that were very close (e.g. IEO $=2$, COS-5 $=3$, Maths residual $=3$, English residual $=2$ ). These schools were an important part of the analysis and examples from their classroom observations are included in the report to illustrate the pedagogical strategies identified. A small group of schools did not fit clearly into any category. Details of these schools, as well as all of the other schools, are given in Appendix 3.

\subsection{The Instruments}

Two observation instruments were adapted and used in the EPPE 3-11 project to obtain information about variation in classroom processes, including teachers' and pupils' classroom behaviour and experiences. The employment of two observation instruments served as a method of exploring validity and reliability and offered the potential for comparison by increasing the range of behaviour covered and by enhancing validity. These instruments were the Classroom Observation System for Fifth Grade (COS-5, Pianta, NICHD, 2001) and the Instructional Environment Observation Scale (IEO, Stipek, 1999). The COS-5 (Pianta) observations were completed in the Spring term (2003/4 and 2004/5) and the IEO (Stipek) observations were conducted in Summer (2003/4 and 2004/5). Visits to schools were made on 'typical' days and over 1,000 children were observed in classrooms across 125 schools. The COS-5 (Pianta) observations were conducted across a range of academic subjects whereas the IEO (Stipek) focused on Literacy and Numeracy only. These instruments were selected because they were devised relatively recently, they are appropriate for the primary age group, they cover a wide range of pupil and teacher behaviours and they offered the opportunity to facilitate comparison with research in other contexts (e.g. Galton et al 1999, NICHD, 1998 and 2001). The following sections provide a brief description of the two instruments.

\subsubsection{The Classroom Observation System for Fifth Grade (COS-5, Pianta)}

The Classroom Observation System (COS-5, Pianta, See Appendix B), developed by Professor Robert Pianta (NICHD, 2001), was initially used by the National Institute of Child Health and Human Development (NICHD) Study of Early Child Care and Youth Development in the USA. The instrument is divided into two main parts that include The Behavioural Coding System, which we refer to as the Frequency of Behaviour Coding System, and the Qualitative Coding System, which we refer to as the Measures of Quality Coding System. These two parts, described in more detail below, make up what is referred to as an observation cycle, lasting 20 minutes in total.

Overall, 1009 observations were conducted in the 125 schools using the COS-5 Pianta instrument. For each school, observers were required to complete a minimum of 8 twenty minute observation cycles. These observation cycles had to include ${ }^{2}$ :

\footnotetext{
${ }^{2}$ This has been adapted from the NICHD (2001).
} 
- One start of the day observation

- One start of the afternoon observation

- Two Literacy observations

- Two Numeracy observations

- One Science or Humanities observation

- One additional academic subject (could be another Literacy, Numeracy, Science or Humanities).

Researchers were therefore asked to schedule all of their observations on days and times when the teacher reported that most teaching, especially of the subjects to be observed (e.g. English, Maths, Science, History, Geography) would be occurring, or what came to be known as 'typical days.'

Table 2.2a presents the number of children observed in each school and the number of cycles and Table $2.2 \mathrm{~b}$ presents the total number of cycles broken down by lesson type.

Table 2.2a COS-5: Number of cycles as a function of number of schools

\begin{tabular}{|l|c|c|c|c|c|}
\hline $\begin{array}{l}\text { No of children observed } \\
\text { (No of 10-min Cycles) }\end{array}$ & 7 & 8 & 9 & 10 & Total \\
\hline Number of schools & 2 & 113 & 9 & 1 & 125 \\
\hline Total & 14 & 904 & 81 & 10 & 1009 \\
\hline
\end{tabular}

Table 2.2b COS-5: Number of cycles broken down by type of lesson

\begin{tabular}{|l|c|}
\hline Curricula subject & Number of cycles \\
\hline Start of the day & 72 \\
\hline Start of the afternoon & 71 \\
\hline Literacy & 153 \\
\hline Numeracy & 149 \\
\hline Science & 76 \\
\hline Humanities & 44 \\
\hline Other & 3 \\
\hline Total (observed in 2004) & 568 \\
\hline Unclassified (observed in 2005) & 441 \\
\hline Total & 1009 \\
\hline
\end{tabular}

Of the 1009 observations, 441 were not classified according to lesson type. However, the proportional representation of each lesson would have been similar to that presented in Table 2 for the 568 observations conducted in 2004 (25\% for Literacy, 25\% for Numeracy and around $13 \%$ for science).

High priority was placed on observing lessons in the core-academic subjects (Literacy and Mathematics) as these are the key academic outcomes in the EPPE research. It was found that the instrument was more difficult and less appropriate to use when applied to non-core 
subjects (e.g. ICT, Music, etc). At the start of each 20-minute observation cycle, observers were required to fill in a cover sheet with general information about the classroom observed. This included information such as the gender of the target child, the lesson observed, the number of adults in the room and their designation (classroom teacher, learning support assistant, etc.), the number of pupils in the class, the type of room (regular classroom, library, ICT suite, etc.) and the time the observation began. A similar page was filled in before the start of the second part of the cycle: The Measures of Quality Coding System.

\section{a) The Frequency of Behaviour Coding System}

Part One of this observation cycle was referred to as The Frequency of Behaviour Coding System and was used during the first of two ten-minute observation segments within the overall COS-5. It included the coding of child and teacher behaviours across a range of classroom and curriculum settings. For the duration of the observation, a target child (TC) was observed and recorded during a sequence of ten 60-second intervals (30-seconds observe, 30-seconds record) during which focus was placed upon capturing information in five general areas of the target child's classroom behaviour and experience. It is important to note that the behaviours included are not exhaustive of the types of behaviours or situations one might observe of a pupil or teacher in a Year 5 classroom.

The categories are:

Child Level Setting - This code is intended to describe the setting in which the target child is working, i.e. whole class, individual or large/small group. In order to identify this, the Research Assistant was asked to focus upon what setting the target child is working in rather than the setting intended by the teacher.

Content of Target Child's Activity - This code is intended to capture the nature of the activity in which the target child is engaged or supposed to be engaged. The codes include subject areas (e.g. Literacy, Numeracy, Science, Humanities etc) as well as non-curricular activities (e.g. Enrichment, Free Time) ${ }^{3}$. The focus here is on the particular activity the teacher has set for the target child during each observation interval. Within the Literacy and Numeracy categories, there were sub-categories which further break down the activity of the target child. The sub-categories under Literacy included 'Word-Level' and 'Comprehension' and under Numeracy, 'Computation' and 'Concept Development/Problem Solving'. Where applicable, these sub-categories could be in conjunction with other subject areas (e.g. if observing a Science lesson where the children were measuring the change in the height of a plant, the Researcher would code Science and 'Computation').

Teacher ${ }^{4}$ behaviour - The codes that fall under the teacher behaviour category focus on a select group of teaching and supporting behaviours, which while comprehensive are not to

\footnotetext{
${ }^{3}$ All Research Assistants were advised to watch academic subject areas rather than 'other' activities. This was ensured by the pre-visit planning with teachers at focal schools to select two appropriate days where this would be possible.

${ }^{4}$ The term 'teacher' here is generic and refers to any adult (e.g. learning support assistants, deputy head, parent volunteers) in the classroom whom the target child had direct contact with during the course of the observation.
} 
be considered an exhaustive list. All the behaviours specify ways in which a teacher may interact with the target child or a group of students that includes the target child. The behaviours include 'Attending to target child' (directly), 'Teaching Basic Skills/Facts', 'Teaching Analysis/Inference', 'Managerial Instructions', 'Monitoring/Checking Work', 'Displaying positive or negative affect' and 'Disciplines'.

Child Academic behaviour - This category includes codes that focus on aspects of the target child's academic behaviour in terms of the intensity and level of involvement demonstrated by the target child (i.e. 'Engaged', 'Highly engaged', 'Unproductive/Spaced Out/Disengaged' or 'Off-task - Alternative Academic Activity'), as well as the type of behaviour the child is engaged in ('Learning/Performing Basic Skills', 'Learning/Performing Analysis/Inference/ Planning', 'Collaborative Work', 'Requesting Attention/Help/Information' and 'Volunteers'). While some of these codes correspond with codes found under the teacher behaviour category, the two were coded independently.

Child Social Behaviour - This category includes codes which capture the target child's social interactions with peers and adults in the classroom, as well as generally disruptive behaviour. These behaviours were coded whenever they were seen occurring and do not have to be characteristic of the full 30 second observation. The codes include 'Positive/Neutral Engagement with Peers', 'Negative/Aggressive Engagement with Peers', 'Positive or Negative Affect Towards Teacher' and 'General Disruptive Behaviour'.

\section{Procedure}

For each COS-5 observation cycle, the researcher focused on a target child. For the duration of the first part of the observation cycle, the researcher watched and coded the target child's behaviour and experience in the classroom for a total of ten sixty-second intervals (30 seconds observe, 30 seconds record) using a standard set of codes (see Appendix B). For the majority of the behavioural categories described above, the Research Assistant was required to choose the one descriptor that best characterised the interval concerned. However, with certain categories, such as 'Teacher Disciplines' or 'Target Child Volunteers', the behaviour would be coded whenever it was observed as occurring (e.g. the target child hits a peer would be coded as 'Negative/Aggressive Engagements with Peers'). These behaviours were called 'events.'

Once the 10 sixty-second intervals were coded, the Research Assistant completed three additional ratings (i.e. Teacher sanctioned classroom setting; Teacher sanctioned collaborative work and Teacher administered test) before continuing to Part Two of the cycle, the Measures of Quality Coding System.

\section{b) The Measures of Quality Coding System}

Part Two of the COS-5 observation cycle was The Measures of Quality Coding System. This was dedicated to ten minutes continuous observation of behaviours and characteristics of the target child, the teacher and any other adults in the room and the classroom environment at a more global level. During this portion of the observation the researcher focused upon the 'who, what and how' of everything happening at the classroom level and with the target child's behaviour within that specific classroom environment. During these final ten minutes the Research Assistant scored each of the 16 constructs (see below) based 
upon the degree to which the behavioural, emotional and physical markers were present and indicative of different levels of each construct. The ratings were completed at the end of the ten-minute observation.

The Measures of Quality Coding System contains two broad categories: Child Codes and Classroom Codes. Under these main headings there are a number of sub-headings or constructs (behaviours, characteristics) that must be rated.

The seven child-level items for rating the target child in the classroom are:

Positive affect

Self-Reliance

Sociable/Co-operative with peers

Attention

Disruptive

Activity level

Child-Teacher Relationship (Main teacher only).

The eight classroom-level items for rating aspects of the overall classroom are:

Richness of Instructional Methods

Over-control

Chaos

Detachment/Teacher

Positive Classroom Climate

Negative Classroom Climate

Purposeful use of instructional time

Evaluative Feedback

Teacher Sensitivity (Main teacher only).

After training, the researcher makes judgements based upon the range of, frequency, intention, and emotional tone of the interpersonal and individual behaviours seen during the observation cycle. Individual items are then rated on a seven-point scale $(1=$ very uncharacteristic and $7=$ very characteristic). After assigning the sixteen qualitative ratings, the observer completes an additional set of codes that rate the teachers on pedagogical strategies.

Training

In order to prepare researchers to use the COS-5 and IEO, intensive training was required. We are grateful to both Robert Pianta and Deborah Stipek who assisted us in informing our training. They provided guidance notes and video material. The training included 12 days of in-house (Institute of Education) training and additional days for researchers to review the materials and practice using the instruments both with videos and in real classroom settings. In addition researchers conducted 'paired' observations to improve reliability. For further information about training and reliability see Appendix 4. 


\subsubsection{Instructional Environment Observation Scale (IEO, Stipek)}

The second instrument used in this part of the research was the Instructional Environment Observation Scale (IEO) designed by Professor Deborah Stipek, University of California, for the Centre on Organisation and Restructuring of Schools. It, like the COS-5 (Pianta, NICHD, 2001) was used to obtain information about variation in school processes, including teachers' and pupils' classroom behaviour and experiences. The purpose of the IEO (Stipek, 1999) is to gather high inference, numerical indicators of the instructional environments experienced by pupils by combining researcher judgements about the teacher's teaching and pupils' learning behaviours. In the EPPE 3-11 study, the IEO was used specifically to observe both Literacy and Numeracy in each of the Year 5 classrooms in 93 focal schools, given the importance of these aspects of the curriculum to later academic success and the development of the National Strategies (DfEE 1999, DfES 2001).

There are 4 main areas under which information was gathered. These include: General Classroom Management and Climate, General Instruction Scales, Mathematical Instruction Scales, and Writing Instruction Scales. Within each of these areas there are a total of sixteen sub-scales (see Appendix 4).

General Classroom Management and Climate Scales:

Classroom Climate

Classroom routines

General Instruction Scales:

Cross-Disciplinary Connections

Linkage to life beyond the classroom

Social support for student learning

Student engagement

Mathematical Instruction Scales:

Use of Maths analysis

Depth of knowledge and student understanding

Basic skill development in the context of problem solving

Maths discourse and communication

Locus of Maths authority

Writing Instruction Scales:

Reading as meaning making

Basic skills development in the context of reading

Higher order thinking in writing

Purposeful development of writing skills

Instructional conversations.

Each of the scales is rated between 1 and 5 , where 1 is low and 5 is high. The following is provided as a 'rule of thumb' for field researchers as they decided upon a rating; 
$1=\quad$ Stereotype of conventional (formal/didactic) and/or undesirable environment.

$2=\quad$ Minimal intensity to mark a shift from the conventional environment could be limited to the teacher or to a few students.

$3=$ Greater and/or uneven intensity in shift from conventional includes some students.

$4=\quad$ Substantial and intense shift from conventional, includes many to most students.

$5=\quad$ Very intense, includes most, to almost all students.

Before the researcher decides upon the rating for each of these scales, s/he is required to take detailed observation notes (field notes). These notes are used as the primary source of evidence upon which the ratings are derived. Researchers are required to review each of the relevant scales prior to beginning an observation in an effort to help them focus their note-taking upon relevant evidence that will help to support their numerical rating. The type of information researchers are encouraged to collect includes teacher and child behaviour, levels of pupil engagement, evidence of how children interact with each other and with the adults in the classroom, the types of questions overheard and the like. One of the benefits of this instrument is that it provides both quantitative and qualitative data upon which analysis can draw. 


\subsection{Data Preparation and coding}

\subsubsection{Identification of key pedagogical strategies}

As noted above, pedagogy was defined broadly in the qualitative analysis to include a range of processes and provisions that could be considered to initiate or maintain learning processes, and to achieve educational goals. The initial qualitative analysis was carried out to identify the application of those pedagogic strategies identified through the EPPE 3-11 classroom observational ratings and accompanying field notes and other strategies identified in a review of the literature. For example, the numeric rating scales and observations provided evidence related to the use of plenary sessions and the practice of building on children's prior experience, and robust evidence was found for assessment for learning in the extant theoretical and empirical research literature.

The first phase of developing the analytical framework of pedagogical strategies involved combining a number of professional focus group discussions with the results of a literature search to ensure breadth and divergence in the identified factors that contribute to effective classroom practice. These were initially grouped into more than 40 strategies and factors, many with similar features. As this list provided many overlaps and some items were duplicated, the list was then collapsed into 11 main strategies with some subheadings. These established pedagogic strategies provided the initial analytic framework that continued to be elaborated through the process of reviewing the literature. We continued to systematically work through the qualitative data until we reached a saturation point (no new strategies emerged) to determine which cases confirmed or confounded the theoretical themes that were emerging. The analytical process was therefore partially 'grounded' and involved inductive processes and constant iteration between the qualitative data, theory and empirical evidence following progressive stages of deduction and verification.

The final list of strategies has been summarised in Table 2.3a below.

Table 2.3a Effective pedagogical strategies

\begin{tabular}{|c|c|c|}
\hline & Category & Pedagogical Strategy \\
\hline 1. & Organisation & $\begin{array}{l}\text { Well organised teaching time, no time wasted, } \\
\text { good pace, good classroom routines, children are } \\
\text { self-reliant }\end{array}$ \\
\hline 2. & Shared Goals & $\begin{array}{l}\text { Clear, shared objectives with specific guidance on } \\
\text { how to achieve them }\end{array}$ \\
\hline 3. & Homework & Use of homework \\
\hline 4. & Classroom Climate & $\begin{array}{l}\text { Positive classroom climate, teacher-child and child- } \\
\text { child interactions } \\
\text { Teacher's in-depth knowledge of, enthusiasm for } \\
\text { and confidence in teaching subject }\end{array}$ \\
\hline 5. & Behaviour Management & Effective behaviour management \\
\hline
\end{tabular}




\begin{tabular}{|c|c|c|}
\hline 6. & Collaborative learning & $\begin{array}{l}\text { Use of groups for a specific, collaborative purpose } \\
\text { Peer tutoring }\end{array}$ \\
\hline 7. & Personalised learning & $\begin{array}{l}\text { Appropriate and considered differentiation } \\
\text { Scaffolding } \\
\text { Variety and richness of teaching resources }\end{array}$ \\
\hline 8. & Making links explicit & $\begin{array}{l}\text { Build on prior pupil knowledge and look forward to } \\
\text { the next steps in the curriculum } \\
\text { Cross and extra curricular links made explicit } \\
\text { Practical teaching activities to teach testing and } \\
\text { application of knowledge and problem-solving }\end{array}$ \\
\hline 9. & Dialogic teaching and learning & $\begin{array}{l}\text { Sustained shared thinking } \\
\text { Open-ended questions used to develop deeper } \\
\text { understanding rather than for summative evaluation } \\
\text { Teaching and encouraging analytical thought } \\
\text { Children's talk encouraged and moderated }\end{array}$ \\
\hline 10. & Assessment for Learning & Ongoing formative assessment \\
\hline 11. & Plenary & Use of plenary \\
\hline
\end{tabular}

\subsubsection{Top down and bottom up analysis}

As stated earlier, the data for analysis came from structured observations which provided numerical ratings and additional qualitative observations made by the researchers (including their field notes) using two classroom observation instruments: The COS-5 and the IEO. The quantitative ratings from the COS- 5 were often able to provide an initial impression of the frequency and application of each of the pedagogical strategies within each 'typical' group of Year 5 classrooms (Group A - academically effective, good quality pedagogy; Group B - medium academic effectiveness and quality of pedagogy and Group C - low academic effectiveness and poor quality pedagogy). Items on the COS-5 that described each strategy were identified (some items were applicable to more than one strategy and so were used more than once) and for some strategies (e.g. homework), none of the COS-5 items applied. Ratings for all schools within a group were combined together to create an overall picture of each group of schools that would allow comparisons to be made between them. Following that, qualitative observations, some from the COS-5, but most from the IEO (because this instrument provided more detailed descriptions, including the researchers' field notes), were used to illustrate the findings.

\subsection{Ethical Considerations}

The EPPSEM study followed the ethical guidelines set in BERA Ethical Guidelines for Educational Research and BERA Quality Conditions for Quality Research. The initial EPPSE project proposal was approved by the Ethics Committee of the Institute of Education. Schools included in this study have been given codes to guarantee their anonymity and teachers and children are never referred to by their real names. Where a child's or teacher's name is used for illustrative purposes, the real name has been changed to a fictitious one. 


\subsection{Limitations of the EPPSEM study}

Limitations of the EPPSEM study include the following:

- As the focus of this study was on classroom strategies, we did not investigate other school and pupil level factors that are known to influence school effectiveness such as leadership, monitoring pupil progress, parental support, attendance, rates of exclusion or inspection evidence. Most of these are addressed elsewhere in the EPPE literature (e.g. see Sammons et al 2006 and 2008).

- Although the large amounts of quantitative data available were used to provide additional support for studying the frequency of use of pedagogical strategies, the main focus of the EPPSEM study was on analysing and interpreting the qualitative descriptions the researchers included in their observations.

- While the study refers to Key Stage 2, observations were only made in Year 5 classes and so results obtained here may not apply across the key stage.

- A constraint of this approach was that the observations focussed on the pedagogical strategies described and measured in the research instruments. This meant that there was sometimes very little data to support or refute some of the strategies identified through the evidence-based literature search and professional focal discussions. 


\section{Section 3 EPPSEM Findings}

This section of the report will present the findings for each of the 11 strategies. For most strategies there is:

- a description of the strategy and where in the data supporting evidence were expected to be found

- an explanation of the main differences and similarities across the three academic effectiveness and quality of pedagogy groups: Excellent, good and poor.

- an exploration of the themes arising from the analysis

- a series of excerpts from the field notes to illustrate good practice

- a summary of the key findings

- a list of pedagogical strategies that distinguish Year 5 classrooms in excellent schools; academically effective schools with good quality pedagogy from the good and poor schools.

\section{Overview}

Most of the teaching and learning observed in the Year 5 classrooms had elements of good pedagogy and some observations recorded very high quality learning experiences. On the whole, children were working in well organised classrooms where they felt safe and respected. The quality of teaching resources was good, teachers were sensitive to the individual needs of their pupils and teacher detachment was low. Pupils were generally wellbehaved and teachers managed behaviour in the classroom effectively. There was some evidence of collaborative work, links to life outside the classroom being made explicit and classroom dialogue aimed at sharing and deepening understanding. About half of these lessons included a plenary session.

A minority of schools (ten schools ( $12 \%$ of schools for which full data were available), poor schools had very low ratings on academic effectiveness and quality of pedagogy. These classrooms were generally chaotic and had poorer quality teaching resources. Teachers had high levels of detachment and the classroom climate was negative. Pupils often misbehaved and teachers seemed to be at a loss about how to cope. Links to life outside the classroom were not made explicit, open-ended questions were rare and children were not encouraged to develop responsibility for their own learning.

The most interesting and effective strategies were found in the ten Year 5 classrooms in the excellent schools that were given the highest ratings for quality of pedagogy and academic effectiveness. These classrooms were very similar to the good schools but had a number of exceptional features. Understanding which characteristics distinguished excellent from good schools is a main focus of this study as teachers and policy makers are often more 
concerned and intrigued by the differences between 'average/good and excellent' than simply 'good and poor or bad' teaching.

Although numerical ratings were available for many of the characteristics of the pedagogical strategies from the different instruments, the data the EPPSEM study draws on are mainly qualitative in nature and so descriptive terms are more appropriate when reporting findings. When numerical scales were available, they have been converted to words and were reported as described in Table 3a.

Table 3a Qualitative descriptions of the numerical ratings

\begin{tabular}{|l|c|}
\hline Numerical Rating & Description \\
\hline Highest ratings on the scale & Very High \\
Ratings in between the highest and the middle rating & High \\
Middle rating on scale & Medium \\
Ratings in between the lowest and middle ratings & Low \\
Lowest ratings on scale & Very Low \\
\hline
\end{tabular}

Four different numerical scales were used on the COS-5 and the IEO and the numerical ratings were matched with appropriate qualitative descriptions (see Table $3 \mathrm{~b}$, below). The cut-off points between the categories were the midpoints between the highest value in the lower group and the lowest value in the higher group.

Table 3b Quantitative scores and their qualitative descriptions

\begin{tabular}{|l|cccc|}
\hline & \multicolumn{5}{|c|}{ Scale ranging from . . } \\
Qualitative Description & 1 to 3 & 1 to 5 & 1 to 7 & 0 to 10 \\
\hline Very High & 3 & 5 & 6,7 & 9,10 \\
High & - & 4 & 5 & $6,7,8$ \\
Medium & 2 & 3 & 4 & 5 \\
Low & - & 2 & 3 & $2,3,4$ \\
Very Low & 1 & 1 & 1,2 & 0,1 \\
\hline
\end{tabular}




\subsection{Organisation}

In order for effective teaching and learning to take place, the primary school classroom environment must be well organised. Teaching and learning time needs to be well planned and routines need to be second nature so that children know what they have to do, how to do it and what to do if they need help.

Both the COS-5 and the IEO provided a great deal of quantitative information about how Year 5 classrooms and lessons were organised. The COS-5 measured how well the teacher's resources were organised and how suitable they were for the particular teaching activity, how productively teaching time was used, how clear the teacher was about what he or she expected the children to do in their activities, how much class time was spent on transitions between activities and on classroom business and the children's self-reliance as well as how much responsibility they had for managing their own time and materials. The IEO provided a further quantitative measure of the effectiveness of classroom routines.

In addition to these quantitative measures, two of the COS-5 quantitative ratings provided an opportunity for the researchers to add their qualitative observations (the target child's selfreliance and how productively teaching time was used) and most researchers did. The IEO required researchers to make observations on the structure, organisation, management, prepreparation and pace of the lesson and the efficiency of the classroom routines and these were good indications of how well-organised the teacher and the classroom were, how wellestablished routines within the classroom were and how well the children understood and met the teacher's expectations. Researchers' field notes of the lessons usually described the teacher's resources and provided detailed illustrations of overall organisation within the classroom including classroom routines, how the teacher managed transitions and other classroom business, ${ }^{5}$ the pace of the lesson and whether or not children were perceived to be self-reliant.

When considering the differences and similarities in classroom organisation, the three groups of Year 5 classrooms originally identified (high academic effectiveness and good quality pedagogy (Group A), medium academic effectiveness and quality pedagogy (Group $B$ ), low academic effectiveness and poor quality pedagogy (Group C)) could be more sensibly reorganised into two: one large group (Groups $A$ and $B$ ) and Group $C$. Groups $A$ and $B$ were found to have very similar ratings on many items and were often rated significantly higher than Group C. Although scores for Group B were lower than those for Group A and higher than those for Group C, they were usually closer to the Group A scores (see Table 3.1a below). On all items, Year 5 classrooms in academically effective schools with good quality pedagogy were rated highest on organisation and those in schools with low academic effectiveness and poor quality pedagogy were rated lowest. Many of these differences were statistically significant $(p<.05)$.

\footnotetext{
5 "Business" refers to administrative and organisational tasks such as taking the register, handing out/collecting in letters or homework, changing from one activity to another (e.g. lining up for assembly).
} 
Table 3.1a Organisation*

\begin{tabular}{|c|c|c|c|}
\hline $\begin{array}{l}\text { Characteristics of Year } 5 \\
\text { classrooms in schools } \\
\text { with . . . }\end{array}$ & $\begin{array}{c}\text { Group A: } \\
\text { Excellent schools }\end{array}$ & $\begin{array}{l}\text { Group B: } \\
\text { Good schools }\end{array}$ & $\begin{array}{l}\text { Group C: } \\
\text { Poor schools }\end{array}$ \\
\hline $\begin{array}{l}\text { Productive use of instructional } \\
\text { time }\end{array}$ & Very High & High & Medium \\
\hline $\begin{array}{l}\text { Teacher's resources are well- } \\
\text { organised and fit for purpose }\end{array}$ & Excellent $^{* *}$ & Very High & High \\
\hline Children's self-reliance & High & Medium & Medium \\
\hline $\begin{array}{l}\text { Teacher's expectations are } \\
\text { clear }\end{array}$ & Very High & Very High & High \\
\hline $\begin{array}{l}\text { Time children spend on } \\
\text { transitions/ management/ } \\
\text { business }\end{array}$ & Very Low*** & Very Low*** & Low $^{* * *}$ \\
\hline Classroom routines & Very High ${ }^{\star * *}$ & $\mathrm{High}^{* * *}$ & Medium \\
\hline $\begin{array}{l}\text { Children are responsible for } \\
\text { time and resources }\end{array}$ & Medium & Medium & Low \\
\hline \multicolumn{4}{|c|}{$\begin{array}{l}\text { Unless otherwise stated, different qualitative descriptions indicate significant differences }(p<.05) \\
\text { between groups. } \\
\text { As the mean score for Group A was equal to the maximum score on this item, and as it was } \\
\text { significantly higher than the mean score for Group B, the additional qualitative description } \\
\text { "excellent" was used for this one item. }\end{array}$} \\
\hline
\end{tabular}

In classrooms in excellent and good schools teacher's lessons and teaching resources were generally well planned and managed and both learning and behavioural objectives and expectations were shared by all members of the class. All of these classrooms were rated highly on how well the teachers had established routines. Children in these classrooms knew what to do, how to do it and how to seek extra help when required. There was a sense of smooth, quick transitions with children moving purposefully from one activity to the next and the result was that very little time was wasted and children were self-reliant. Some descriptions that typified these well-organised classrooms came from the IEO reports of two classrooms good schools:

Transitions are routine for almost all the children. The teacher packs a lot into the lesson. Everyone knows what is expected of them. (S26, Literacy IEO).

Classroom Routines: No time was wasted in moving from set literacy table places to the carpet at the start and the end of the lesson. The teacher was very well prepared with an OHP of the poem and interactive board sentences and the worksheets. The teacher's expectations are very clear as to what to do and what time they have to complete the task. The TA (teaching assistant) role is with a group of 8 children - less able; this is clearly defined, and they (the teacher and the TA) have planned prior to lesson. Equipment all available. (S28, Literacy IEO)

The phrase "no time wasted" was repeated over and over, particularly throughout the observations of academically effective schools with good quality pedagogy. It was clear that children in these classrooms were completely familiar with the classroom routines and that the routines themselves had been designed to minimise disruption and maximise teaching 
time. In these classrooms, when children were occasionally 'off task' or when disorganisation or chaos did occur, teachers quickly regained control so that the lesson could move forward.

Year 5 teachers in excellent schools excelled at preparing and organising suitable, high quality teaching resources for their lessons. They were also able to use the available teaching time more effectively and these two characteristics, combined with the higher degree of self-reliance of the children in their classrooms, appear to be key components in achieving excellence in this area.

Although Year 5 classrooms in poor schools were rated as medium or good on most items, their scores were significantly lower than those of the two other groups on how well the teacher's resources were organised and how fit for purpose they were, how productively instructional time was used, the clarity of the teacher's expectations, including classroom routines, and the children's independence and self-reliance. A typical description of a classroom in one of these schools was:

Time is not well managed. Lessons run over leaving no time for the next lesson.

Organisational difficulties in these classrooms were evident right from the very beginning of lessons. Instead of a crisp, sharp start, lessons began slowly and without any sense of urgency or brisk pace. In one school, for example, the teacher appeared to be resigned to a slow and disorganised start to the lesson and apparently did not see the need to change this:

... children take a long time to respond and are often late arriving for sets.

Teacher does not seem to mind this. (S76, Numeracy IEO)

Even when good planning did take place and the teacher put a lot of effort into effective delivery, lessons in these Year 5 classrooms were observed to be disorganised. One Year 5 teacher, despite good planning for his lesson and a great deal of effort on his part, was unable to establish control of his class or a good teaching pace. The teacher "strives for control and to make himself heard" (S79, Numeracy IEO). The researcher noted:

He can get involved with individuals and the SEN group but then becomes completely detached. He shouts for control then ignores the behaviour for long periods. His presentations are very hurried as though "This is what I said I'd do and I'm going to do it" but once he's done that, he doesn't really seem to care about the children's work. He doesn't monitor children's work in any depth nor is there feedback. (S79, Literacy IEO)

When reading the field notes of the lesson, the teacher's losing battle and increasing despair are obvious. The reader can feel his desperation to maintain control and to teach his pupils something/anything but the inevitable failure of his efforts is obvious from the start. The overall picture in these classrooms was one of inadequately prepared resources, poor 
understanding of both behavioural and learning expectations, general disorganisation and as a consequence, children who were not encouraged to function independently.

Year 5 children in excellent schools were rated as significantly more self-reliant $(p<.05)$ than their peers in other schools whereas there were no significant differences between good and poor schools on this aspect.

A surprising finding was that none of the groups differed significantly on the amount of time teachers or children spent on classroom business and management activities (e.g. taking the register, lining children up for Assembly). Although children in poor schools spent a little more time than their peers on management and classroom business activities, the amount of time spent by any of the groups on these mainly administrative tasks was small overall and there were no significant differences between any of the groups. The similarity between the groups on the amount of time spent on 'business' may have been because many of the observations where this was recorded took place at the beginning of lessons, when teachers spent roughly equal amounts of time on 'business'. Perhaps if an overall measure had been included on the IEO, differences between the groups might have been found on this aspect as in other research of effective teaching (Mortimore et al., 1988).

In summary, children in excellent schools were working in very well-organised classrooms. They were clear about what they needed to achieve in their lessons and how to do it. They were encouraged to be self-reliant and frequently responsible for managing their own time and resources. Nearly $100 \%$ of observations rated these teachers' lessons as well-planned with all required materials fit for purpose and organised before the start of the lesson. There were relatively few instances of chaos and almost all instructional time was carefully planned and well used. Good schools closely mirrored excellent schools with some important exceptions in three areas: i) resources that were well organised and fit for purpose, ii) the productive use of instructional time and iii) encouraging children's self-reliance, where they were rated lower than highly academically effective schools.

What does good organisation look like in Year 5 classrooms?

- From the very beginning a quick pace. No time lost whatsoever ( S01, Literacy IEO:3)

- Difficult to see how any more could have been squeezed in (S01, Numeracy IEO:3)

- No sense of time wasted (S01, COS-5 (2):5)

- Children immediately sit on carpet as arrive in room. Routine to brainstorm in pairs then as class went smoothly (S02, Literacy IEO:3)

- Little time wasted. Children quickly settled to the task and a high percentage were on task (S03, COS-5 (3):3)

- No time lost. Very quick start to Literacy . . . Finally all cleared up quickly. They know what to do and they do it (S04, Literacy IEO:3)

- Routines are very efficient - have monitors for everything to help the teacher. No disruption (S05, COS-5 (7):5)

- Well established routine (S06, COS-5 (1):5)

- Children familiar with routines - they know what to do when they return from assembly (S06, Numeracy IEO:3) 
- Couldn't be smoother (S07, Literacy IEO:3)

- A well oiled machine. Happy confident children move easily from one activity to the next (S07, Numeracy IEO:3)

- No time lost ever ... Great routines which all follow (S08, Literacy IEO:5)

In poor schools, children often did not understand what they were supposed to do or how to do it, were less self-reliant and had less responsibility for managing their own time and resources. Teachers in these classrooms had resources that were not well-prepared (not enough copies, computer/online resources that didn't work), changed their minds sometimes mid-sentence - about what they wanted the children to do or provided activities whose sole purpose seemed to be filling up time. Although levels of chaos in poor schools were not consistently high, some schools had very high ratings on chaos and overall ratings of chaos were significantly higher in these schools than in the other two groups. Finally, in poor schools, only about $1 / 3$ of observations rated that time was being managed efficiently around teaching and learning.

Key findings on organisation:

1. Organisation in Year 5 classrooms in excellent and good schools was judged to be at least good and often better. On most items, at least some Year 5 classrooms in these groups received the highest possible rating and sometimes none received the lowest possible rating.

2. Although there were some exceptions (teachers' resources, clarity of teachers' expectations), organisation in poor schools was generally judged to be average or below. For some items, these classrooms received the lowest possible ratings and for others, none of the schools in this group received the highest possible rating.

Year 5 teachers in excellent schools excel at:

- Providing high quality teaching and learning resources: Teachers provided highquality teaching resources thoughtfully adapted to meet the needs of their pupils.

- Making effective use of time: Every second counts. No time was wasted.

- Encouraging self-reliance: Children were responsible for their own time and resources. They knew what to do and they did it independently. 


\subsection{Clear, shared objectives}

Good teaching and learning are facilitated by teachers and pupils working towards common, shared goals that are agreed and understood by all concerned. Children need to know what they are supposed to be learning and how much they should aim to achieve over a defined period of time and they need to internalise these goals as their own. In order for children to be able to achieve their learning goals, teachers must ensure their pupils understand the learning objectives and have a good understanding of the key learning concepts and ideas required to meet these goals.

The COS-5 included two quantitative measures of how well goals were shared and whether or not teachers ensured that key concepts and ideas were clear to their pupils. In addition to these, both the COS-5 and IEO qualitative observations provided an insight into whether or not teachers explicitly shared their learning objectives with their pupils and how thoroughly they checked their pupils' understanding of key ideas. The field notes of the lessons, once again, gave further information about how well the teacher made the lesson objectives and key learning concepts clear to the children. Researchers often noted whether the learning objectives were written up on the board for the children and whether or not the teacher referred to them during the lesson. Teachers' explanations and approaches to new ideas and concepts, how they handled any difficulties or misconceptions and the children's reactions to these, were often described in detail.

The Year 5 classrooms were again split between the three groups. Teachers in excellent schools were rated most highly on sharing learning goals with their pupils. Good schools' teachers were rated significantly higher than poor schools' teachers both on making sure the learning intentions of their lessons were clear to their pupils and on how thoroughly they ensured that key learning concepts and ideas were clear to their pupils.

Table 3.2a Shared Goals*

\begin{tabular}{|c|c|c|c|}
\hline $\begin{array}{l}\text { Characteristics of Year } 5 \\
\text { classrooms in schools with . . }\end{array}$ & $\begin{array}{c}\text { Group A: } \\
\text { Excellent schools }\end{array}$ & $\begin{array}{c}\text { Group B: } \\
\text { Good schools }\end{array}$ & $\begin{array}{c}\text { Group C: } \\
\text { Poor schools }\end{array}$ \\
\hline $\begin{array}{l}\text { The learning intentions of the } \\
\text { lesson or activity are clear to } \\
\text { all children }\end{array}$ & Very Good & Good** & Good** $^{*}$ \\
\hline $\begin{array}{l}\text { The teacher ensures concepts } \\
\text { and ideas are clear to all } \\
\text { children }\end{array}$ & Very Good & Very Good & Good \\
\hline
\end{tabular}

In excellent and good schools, almost all teachers were rated extremely highly on both ensuring that the lesson objectives were clear and that concepts and ideas were clear to the children. They did this by (usually) writing the lesson objective on the board and by making sure that pupils understood what they were expected to achieve in each lesson. It was standard practice for lesson objectives to be up on the board, for teachers to check that 
children understood the main ideas of the lesson and for them to intervene when understanding was not clear or complete.

The picture in excellent and good schools was one where teacher and pupils were absolutely clear about what should be happening at all times combined with a commitment on both parts to ensure that goals were achieved. In excellent schools, teachers were especially good at ensuring the objectives of the particular lesson or activity were clear to every child.

What does ensuring learning objectives, concepts and ideas are clear look like in Year 5 classrooms?

In S02, the teacher changed her lesson when she realised her pupils had not grasped one of the main principles needed to complete a symmetry activity. After an introduction to symmetry (the children had been working on this topic in Art as well as in Maths), the children were asked to work in pairs and create patterns for each other to repeat. The patterns had to contain two lines of symmetry and a perpendicular line. Despite a careful introduction to the task and the teacher modelling how to approach it, the children were still not sure what to do (there was much confusion over how to draw a perpendicular line). When the teacher realised that the confusion was wide-spread, she stopped the lesson, reexplained the task and then asked the children to have another go (S02, Numeracy IEO).

In contrast, a commitment to a common goal was missing from many of the poor schools' classrooms. Teachers were slow to check, and to correct where necessary, their pupils' understanding of key concepts and ideas. Although children in these classrooms were aware of any lesson objectives, it was not clear whether or not they fully understood them or how to achieve them and there was much less focus and drive to meet these goals.

Key findings on shared goals:

1. Most Year 5 teachers, regardless of the academic effectiveness or quality of pedagogy of their school, ensured that the learning intentions of their lessons were shared with their pupils, although teachers in poor schools were not rated as highly on this as Year 5 teachers in other groups.

2. Year 5 teachers in excellent and good schools were better at ensuring that their pupils understood both the learning intentions and the ideas and concepts presented in their lessons.

Year 5 teachers in excellent schools excel at:

- Providing clear learning objectives: teachers ensured that each child knew what he or she was expected to learn during each lesson and activity. 


\subsection{Homework}

Although details about homework were not required to be recorded on either the COS-5 or the IEO, some references to homework were found in the data. However, whether or not homework was a regular expectation in each classroom, how it was used and the quantity and quality of homework assigned could only be guessed at by looking at any references to homework that were included in the observation records.

Homework was only mentioned in three of the excellent schools and appeared to serve two purposes. It was used to provide children with opportunities to learn and practise basic skills and learning spellings was, in fact, the most commonly mentioned homework. Presumably, by asking children to complete these activities at home, class time could be freed for other learning activities. Teachers in these classrooms also set homework that was directly linked to what the children were learning in class, either as a way of providing extension work or, again, to allow more class time to cover other topics or complete other activities. They took care to explain the homework so that children could get the most out of the work set.

In the observations of Year 5 classes in good schools, formal homework was only referred to in one school (S27). In this school, the homework was used to clarify some teaching points. The children were learning how to measure irregular shapes and the teacher used homework that had been set to clarify and consolidate pupils' understanding. In two other schools, although there was no direct mention of homework, pupils were set some optional work to be done outside class time. In S30, a bottom set literacy group had been working on the 'true' story of The Three Little Pigs. The children were asked to think about whether they believed the wolf's or the pigs' side of the story before the next lesson. Children in S31 had really enjoyed a poetry lesson that centred on alliteration and on a combination of collaborative and individual work that was brought together during the plenary. The teacher offered a house point to anyone who could write another verse of the class poem during their break time.

Although the homework in the last two cases was very informal, it was highly likely that at least some of the children would take up these opportunities to extend the work done during the lesson. In the first case, the task was an interesting extension to the class activity and was easily achievable by this group of pupils who struggled with literacy. Children in the poetry lesson were highly engaged and really enjoyed writing their poems so the opportunity to continue with the task, combined with the incentive of a house point, meant they were very likely to take on the challenge.

This informal approach also meant that the confrontation that may arise over incomplete homework was avoided. Children who completed the tasks would be rewarded (by praise, by being better prepared for a class discussion and by being awarded a house point) but those who didn't would not be singled out for punishment. While it could be argued that these tasks weren't really homework and the informality of them was due more to the teachers' laziness (no marking, no consequences to follow through on) or were simply the result of an unplanned moment of opportunity seized by the teacher, setting work to be done outside of class time in this way meant that all children had an equal opportunity to take 
advantage of it and the situation where some children may be reprimanded for not doing their homework was completely avoided.

In the poor schools' classrooms, the attitude of teachers towards homework was more often that they were setting it simply because they were required to set it. These teachers were very concerned with the actual setting and collecting in of homework (and they gave out consequences when homework was not complete) but the work itself was rarely linked to what the children were learning in class. Further, there were no examples of teachers using opportunities that arose during a lesson to set different/more homework than what was already planned.

Finally, although the observation data from the IEO and COS-5 could not provide evidence of the teachers' intentions regarding the homework they set, the teacher survey of the 125 schools from which these schools were selected did find that where there was more emphasis on homework (according to teachers), the EPPE pupils made more progress (Sammons et al., 2008).

\subsection{Classroom Climate}

Both the COS-5 and IEO instruments provided a wealth of information about the classroom climate. Each instrument had a 'positive classroom climate' subsection that offered good insight into the relationships between teachers and pupils. In addition, the first page of the COS-5 (especially 'teacher behaviour: shows positive affect' and 'child social behaviour: positive affect towards teacher'), the 'Child Codes' section of the instrument (especially 'positive affect (child-teacher)' and 'the children are liked and respected by peers') and the 'Classroom Codes' page (especially 'over control', 'detachment/teacher' and 'teacher sensitivity') provided further opportunities for researchers to record their observations of the relationships and environment within the classroom. In addition to all of this, the field notes the researchers kept of the lessons for the IEO ratings provided a useful source of information about the overall feeling of each classroom.

Four main themes emerged from the analysis of the data on classroom climate: (i) overall classroom climate, (ii) child-teacher relationship, (iii) teacher support and (iv) a safe and supportive environment.

\subsubsection{Overall Classroom Climate}

The overall picture regarding classroom climate was very clear. Excellent schools had the highest rating on positive classroom climate, followed by good schools, and excellent and good schools both had very low ratings on negative classroom climate. Poor schools were rated significantly lower on positive classroom climate and higher on negative classroom climate than the other groups. 
Table 3.4a Overall Classroom Climate*

\begin{tabular}{|l|c|c|c|}
\hline $\begin{array}{l}\text { Characteristics of Year 5 } \\
\text { classrooms in schools with . . }\end{array}$ & $\begin{array}{c}\text { Group A: } \\
\text { Excellent schools }\end{array}$ & $\begin{array}{c}\text { Group B: } \\
\text { Good schools }\end{array}$ & $\begin{array}{c}\text { Group C: } \\
\text { Poor schools }\end{array}$ \\
\hline Positive Classroom Climate & Very High & High & Medium \\
\hline Negative Classroom Climate & Very Low & Very Low & Low \\
\hline
\end{tabular}

Unless otherwise stated, differences in qualitative descriptions indicate significant differences $(p<.05)$ between groups.

The minimum and maximum ratings given to schools in the different categories of academic effectiveness and quality of pedagogy, as well as the positions of their means relative to each other and to their actual places on the scales, illustrate the differences between the groups almost perfectly. None of the poor schools' classrooms received the highest possible score for positive classroom climate on either of the instruments however some received the lowest possible score. In excellent and good schools, none received the lowest score on positive classroom climate and some schools in both categories received the highest score. In addition, on the IEO, all of excellent schools scored at least the median (scale 1-5, median $=3$ ). Pupils in these classrooms worked in a positive classroom climate in all the observed sessions.

When looking at the means for positive classroom climate, the mean score in poor schools were just below the median on both scales (COS-5: median $=4$, mean $=3.50$; IEO: median $=3$, mean $=2.57$ ) indicating that positive classroom climate was often missing and, in general, only approached the median rating. In contrast, mean ratings for excellent and good schools were towards the top end of the scales. Good schools had a mean rating between the top two scores on both scales (COS-5: $\max =7$, mean $=6.25$, IEO: $\operatorname{Max}=5$, mean $=4.75$ ) and those in good schools had a mean rating towards the top of the scale (COS-5: scale 1-7, mean $=5.47$; IEO: scale 1-5, mean $=4.06)$. This indicates, once again, that pupils in these classrooms were most likely to experience a positive classroom climate.

The reverse was true for scores on negative classroom climate on the COS-5 and overall ratings were significantly higher for classrooms in poor schools than ratings for excellent and good schools (there was no significant difference between these last two groups). Although the mean rating for negative classroom climate for poor schools was just below the midpoint (median $=4$, mean $=3.21$ ), the mean scores for excellent and good schools were only just above the lowest possible score $(\mathrm{min}=1$, mean (medium academic effectiveness) $=1.30$, mean (high academic effectiveness) $=1.14$ ) and so the difference between them is striking. Poor schools scored near the average rating whereas in excellent and good schools, there were almost no instances of any negative classroom climate. This difference of 'some' versus 'none' seems to be very important.

In summary, the overall observed classroom climate in poor schools was often rated as very poor. Pupils in these classrooms were less likely to experience a positive classroom climate and more likely to experience a negative classroom climate. Pupils in excellent and good schools were generally learning in a positive classroom climate and those in excellent schools were most likely to experience a positive classroom climate. 


\subsubsection{Child-Teacher relationship}

The relationships between teachers and their pupils were measured in several ways. Instances of positive or negative interactions were recorded on the first part of each observation cycle on the COS-5 (teacher displays positive/negative attitude towards target child, target child displays positive/negative attitude towards teacher). A further overall measure of the relationship between the teacher and the target child (child-teacher relationship) was included on the second part of the COS-5 cycles. This second measure also allowed researchers to record their qualitative observations. Once again, the field notes the researchers took as part of their IEO records provided excellent descriptions of the relationships between pupils and their teachers.

Table 3.4b Child-Teacher relationship*

\begin{tabular}{|l|c|c|c|}
\hline $\begin{array}{l}\text { Characteristics of Year 5 } \\
\text { classrooms in schools with }\end{array}$ & $\begin{array}{l}\text { Group A: } \\
\text { Excellent }\end{array}$ & $\begin{array}{c}\text { Group B: } \\
\text { Good schools }\end{array}$ & $\begin{array}{c}\text { Group C: } \\
\text { Poor schools }\end{array}$ \\
\hline Teacher displays positive affect & Very Low & Very Low & Very Low \\
\hline Teacher displays negative affect & Very Low & Very Low & Very Low ${ }^{* *}$ \\
\hline Target child displays positive affect & Very Low & Very Low & Very Low \\
\hline Target child displays negative affect & Very Low & Very Low & Very Low \\
\hline Child-Teacher Relationship & High & Medium & Medium \\
\hline
\end{tabular}

Unless otherwise stated, differences in qualitative descriptions indicate significant differences $(p<.05)$ between groups.

** Although all scores on teacher displays negative affect were very low, Group C teachers scored significantly higher on this item than teachers in Groups A or B.

Although displays of positive or negative interactions between teachers and children were rarely recorded on the first part of each COS-5 cycle (this explains why all of the ratings are very low), the fact that a significant difference was found between the groups on the number of times teachers displayed negativity is telling. Poor school's teachers were observed to be more likely to display negativity towards the target child than teachers in either of the other two groups. The most compelling numerical evidence comes from the minimum and maximum scores for each group. Displays of negativity never occurred in the observations of excellent schools $(\max =0)$ and in good schools, the maximum number of times researchers observed a teacher displaying negativity during an observation was only once $(\max =1)$. In contrast, in poor schools, the maximum score was much higher (scale $0-10$; $\max =5$ ). This shows a very different picture between schools at the bottom end and schools towards the middle and the top end of the group. Pupils in poor schools were more likely to experience disapproval or reprimands or an expression of the teacher's dislike than pupils either of the other groups.

The COS- 5 overall measure of the child-teacher relationship showed that while relationships between the target children and their teachers were good on the whole, those between children and teachers in excellent schools. In excellent school classrooms, the researchers often commented on the affection shown by teachers towards their pupils and affection shown by the teacher was a key feature of the classrooms observed. The teachers seemed happy to be in their classrooms with their pupils and to genuinely enjoy the time they spent with them. One researcher commented: "Happy and relaxed children . . C Children smile 
lots at the teacher - she has a lovely, jokey attitude and obvious fondness for the children" (S02, Literacy IEO). Children and teachers in these classrooms genuinely liked each other and this was a large part of the overall feeling in the class.

\subsubsection{Teacher support}

In order to effectively support their pupils, teachers need to be fully involved with them and sensitive to their needs. The COS- 5 and IEO provided three quantitative measures of teacher support (see Table 3.3c, below). The COS-5 had measures for teacher detachment and teacher sensitivity and there was also the option for researchers to record qualitative evidence to support their numerical judgments. The Literacy IEO had an item on social support for student learning in Literacy and the field notes contained many descriptions of teacher support or, in some cases, the lack of it.

Table 3.4c Teacher support

\begin{tabular}{|l|c|c|c|}
\hline $\begin{array}{l}\text { Characteristics of Year 5 } \\
\text { classrooms in schools with . . }\end{array}$ & $\begin{array}{c}\text { Group A: } \\
\text { Excellent schools }\end{array}$ & $\begin{array}{c}\text { Group B: } \\
\text { Good schools }\end{array}$ & $\begin{array}{c}\text { Group C: } \\
\text { Poor schools }\end{array}$ \\
\hline Teacher detachment & Very Low & Very Low & Low \\
\hline Teacher sensitivity & Very High & High & Medium \\
\hline $\begin{array}{l}\text { Social support for learning in } \\
\text { Literacy }\end{array}$ & High & High & Medium \\
\hline
\end{tabular}

Unless otherwise stated, differences in qualitative descriptions indicate significant differences $(p<.05)$ between groups.

The overall picture regarding teacher support was encouragingly good (low detachment, high sensitivity and high social support for learning in literacy) however the Year 5 classrooms were again split between the three groups. Teachers in poor schools were rated significantly higher on teacher detachment and significantly lower on sensitivity and social support for student learning in Literacy than the teachers in the two other groups. There were no significant differences between the teachers in excellent and good schools on teacher detachment and social support for learning in Literacy. This demonstrates that most Year 5 teachers are involved with their students and provide a high level of social support for their learning.

Teachers in excellent schools stood out by demonstrating significantly more teacher sensitivity than either of the other two groups. Although teachers in good schools were significantly more sensitive to the needs of their pupils than those in poor schools, the teachers in excellent schools scored the highest. Excellent schools' teachers knew their pupils exceptionally well and adapted their approach and activities to suit them. They also provided considerable and consistent encouragement to the children and this was most often demonstrated through the frequent use of appropriate praise. The way in which teachers praised the children communicated both genuine respect for the child's achievements and encouragement for the child to continue to improve his or her work.

Respect, between teachers and children, and as a significant part of the classroom ethos, was evident from the researchers' observations of all of the classrooms. These were classrooms where children's opinions and feelings were valued and where they were expected to respect the opinions and feelings of everyone in the classroom. Instances of 
discipline problems were rare and, when they did occur, were sensitively handled by the teacher without belittling the child.

One example of a teacher sensitively handling a situation where a child was not on task occurred during one of the S08 observations. The researcher noted that the target child started the lesson looking "glum" (S08, COS-5 (2):4). The teacher sat next to him for part of the observation to make sure that he was on task and participating in the group work. The researcher noted that the child was very attentive during the time the teacher was with his group. The teacher also questioned him about the work, smiled at him and used "positive body language", presumably to encourage him to participate fully in the lesson.

The overall impression of the teachers' attitudes towards the children and the level of support and concern provided were overwhelmingly positive. These teachers appeared to know their pupils well, to provide a safe, supportive and encouraging learning environment and to communicate their concern and genuine affection for the children clearly.

\subsubsection{Safe, supportive environment}

Children learn best in an environment in which they feel safe and supported and where there is a culture of mutual respect. The COS-5 provided a number of quantitative measures (target child displays positive/negative affect towards peers, target child demonstrates positive affect, target child is sociable/cooperative with peers, children are well-liked and respected by peers) as well as some qualitative evidence that, along with the field records from the IEO, gave good insight into how safe and well-supported children felt in their classrooms (see Table 3.3d, below).

Table 3.4d Safe, supportive environment*

\begin{tabular}{|l|c|c|c|}
\hline $\begin{array}{l}\text { Characteristics of Year 5 } \\
\text { classrooms in schools with . }\end{array}$ & $\begin{array}{c}\text { Group A: } \\
\text { Excellent schools }\end{array}$ & $\begin{array}{c}\text { Group B: } \\
\text { Good schools }\end{array}$ & $\begin{array}{c}\text { Group C: } \\
\text { Poor schools }\end{array}$ \\
\hline $\begin{array}{l}\text { Target child demonstrates } \\
\text { positive affect }\end{array}$ & High & High & Medium $^{* *}$ \\
\hline $\begin{array}{l}\text { Target child displays } \\
\text { positive/neutral affect } \\
\text { towards peers }\end{array}$ & Low & Low & Low \\
\hline $\begin{array}{l}\text { Target child displays } \\
\text { negative/aggressive affect } \\
\text { towards peers }\end{array}$ & Very Low & Very Low & Very Low \\
\hline $\begin{array}{l}\text { Target child is sociable/ } \\
\text { cooperative with peers }\end{array}$ & Medium ${ }^{* * *}$ & Medium & Medium $^{* * *}$ \\
\hline $\begin{array}{l}\text { Children are well-liked and } \\
\text { respected by peers }\end{array}$ & Very High & High & Medium $^{*}$ \\
\hline
\end{tabular}

Unless otherwise stated, differences in qualitative descriptions indicate significant differences $(p<.05)$ between groups.

** Although they fall into different qualitative categories, the differences between Group $C$ and the other groups are not significant.

*** Although all three groups fall into the same qualitative category, classes in Group $C$ are at the low end of this category and those in Group A are at the high end of this category and the difference between these two groups is significant $(p<.05)$. 
Children appear to be equally happy in their classrooms and with each other regardless of the levels of academic effectiveness or quality of pedagogy of the school. There were no significant differences between the groups on the measures of the affect the target child displayed in general or towards his or her peers. In all groups, children showed much more positive attitudes towards their peers than negative. There was, however, a difference in the level of sociability and cooperation between the observed target child and his or her peers. Although not all differences were significant, target children in excellent schools were significantly more sociable and cooperative than their peers in poor schools.

The teachers in excellent schools showed consistent respect, social support and concern for their pupils. This was demonstrated through the nature of their relationships with their pupils and in the relationships pupils had with each other as these in turn were shaped by the classroom ethos and the example set by the teacher. The degree to which pupils felt safe both physically secure and safe enough to take risks associated with their learning (e.g. risking a wrong answer) - was a good indication of how well supported pupils felt.

Observations indicated that how highly well liked and respected by their peers the children in excellent schools were. Children in good classrooms were rated significantly higher on this item than children in poor schools however children in excellent schools' classrooms were consistently rated the highest $(p<.01)$. In their qualitative descriptions, researchers regularly referred to the safe and supportive feeling within these classrooms and how this seemed to facilitate the children's confidence and approach to their learning. There appeared to be a genuine feeling of mutual trust that helped children to concentrate their energy on their learning and to take risks that would further their understanding.

What does a positive classroom climate look like in excellent Year 5 classrooms? These are happy, safe industrious classrooms: The overall feeling in the classroom is a combination of a strong work ethic and a positive, supportive approach. Relationships between all members of the classes were respectful and supportive.

- A feeling that children were enjoying their learning and confident. A feeling of respect between children and teacher ... . Very aware of children. A very warm supportive attitude, which reflects a child-centred approach. No need to discipline (SOI 1, COS-5 (1):5)

- An experienced teacher, firm, friendly with high expectations (S01, Literacy IEO:3)

- Classroom climate ... a strong feeling of respect for each other. A mature attitude (S01, Literacy IEO:5)

- The teacher was supportive and encouraging, smiled a lot (S01, Literacy IEO, field notes:4)

- Firm but friendly and supportive teaching approach. . The children feel very secure in terms of both learning and socially (S01, Numeracy IEO:3)

- Supportive approach with high expectations (S01, Numeracy IEO, field notes:3)

- (The teacher) is happy and jokey with the rest of the class. Lots of smiles are shared. Mentors different groups - praise-encouragement. Obvious affection seen (S02, COS-5 (6):6) 
- Happy and relaxed children ... Children smile lots at the teacher - she has a lovely jokey attitude and obvious fondness for the children (S02, Literacy IEO:3)

- Warm supportive feeling. Children very content (S03, COS-5 (8):5)

- The classroom seemed totally safe. The children were confident and comfortable and enjoying each other's company; also respectful towards the teacher who they obviously liked (S03, Literacy IEO:3)

- The teacher established firm, friendly control and the children responded. They clearly enjoyed their learning and all were respectful. Lots of praise, encouragement for the children (S03, Numeracy IEO:3)

- Teacher is very involved and aware of children. Lots of praise, e.g. "fantastic"” (S04, COS-5 (3):5)

- A very safe classroom, the children are secure and confident. The teacher maintains firm, friendly and supportive control. All respect each other - adults, children. The children are enjoying their learning. Nothing less than a 5 (maximum score) (S04, Literacy IEO:3)

- A lot of support for the children from the class teacher, the special needs teacher and the learning support assistant. Children were also supportive to each other (S04, Literacy IEO:3)

- All (classroom climate) characterised by mutual respect (S04, Numeracy IEO:3)

- Teacher praises the way they are all getting on with each other (Target child) cooperates all tasks all session (S05, COS-5 (3):4)

- Good relationships seen, affectionate (S05, COS-5 (5):5)

- Demeanour of teacher: relaxed, does not raise her voice, lots of smiles . . l laughed with children at the news reader reports. Very proud of children's achievements (S05, Literacy IEO:3)

- Relaxed, well prepared. . Never raised voice. Lots of smiles and pats on back to children (S05, Numeracy IEO:3)

- Genuine respect by children and teacher for all in the class (S06, COS-5 (4):5)

- Good evidence of mutual support between children. Class teacher and teaching assistant are both encouraging and listen respectfully to children's suggestions (S06, Literacy IEO:3)

- This classroom is a safe place to take risks (S07, COS-5 (7):5)

- Such mutual respect allows children to confidently share their writing with the class. Everyone listens intently as others read. Class teacher makes positive comments but also some fair criticisms well received (S07, Literacy IEO:3)

- Respect obvious. Work well in groups - negotiate roles, share work and help others (S08, Literacy IEO:5)

- A safe place to make mistakes (S07, Numeracy IEO:3)

- Lovely atmosphere - children laugh and smile (S08, COS-5 (2):5)

- Obvious affection for children (S08, Numeracy IEO:3)

In summary, all of the classrooms in excellent schools were consistently rated very highly on the respect, social support and concern the teachers showed for their pupils. Indicating a very positive, supportive learning climate. Researchers commented in their notes about the obvious affection of teachers for their pupils, the mutual respect between all members of the classroom community and how this created a happy, productive learning environment. Both instruments were filled with observations of the teachers' caring and supportive role and one 
quotation succinctly summed up the overall safe and encouraging feeling at the centre of the relationships within these classrooms:

"Very positive feeling. A quiet buzz of work. All respect each other" (S03, $\cos -5(1))$

Key findings on classroom climate:

1. Classroom climate was much more positive in Year 5 classrooms in schools with medium and high academic effectiveness.

2. Teachers were more detached and there was a greater level of over control in Year 5 classrooms in academically less effective schools.

3. Teacher sensitivity (to pupils' individual needs and interests) and children's respect for each other was greater in Year 5 classrooms in schools with medium and high academic effectiveness.

4. Children in Year 5 classrooms in schools with medium and high academic effectiveness were more engaged.

Year 5 teachers excellent schools excel at:

- Providing an extremely positive classroom climate

- Sensitively responding to the needs of their pupils

- Creating an environment where children are well-liked and respected by their peers. 


\subsection{Behaviour Management}

Effective behaviour management within the classroom is an important necessary condition for successful teaching and learning. Teachers and children need to feel safe and to work in an environment conducive to learning in order for pupils to make good progress.

The COS-5 provided a number of quantitative measures of behaviour management within the Year 5 classrooms: how often the teacher had to discipline the target child, measures of the target child's level of disruptive behaviour and the levels of over control and chaos within the classroom. In addition, researchers could record qualitative observations on the COS-5 about the target child's level of disruptive behaviour and levels of over control and chaos within the classroom. The IEO contained a measure of the children's engagement with their learning and the field notes that accompanied the IEO were another good source of information about how behaviour was managed within the Year 5 classrooms (see Table $3.5 a)$.

Table 3.5a Behaviour Management*

\begin{tabular}{|c|c|c|c|}
\hline $\begin{array}{l}\text { Characteristics of Year } 5 \\
\text { classrooms in schools with . . }\end{array}$ & $\begin{array}{c}\text { Group A } \\
\text { Excellent schools }\end{array}$ & $\begin{array}{l}\text { Group B: } \\
\text { Good schools }\end{array}$ & $\begin{array}{c}\text { Group C: } \\
\text { Poor schools }\end{array}$ \\
\hline Teacher disciplines & Very Low & Very Low & Very Low** \\
\hline $\begin{array}{l}\text { Frequency of target child's } \\
\text { disruptive behaviour }\end{array}$ & Very Low & Very Low & Very Low \\
\hline $\begin{array}{l}\text { Target child's overall level of } \\
\text { disruptiveness }\end{array}$ & Low & Very Low & Low \\
\hline Engagement & High & High & Medium \\
\hline Over control & Very Low & Very Low*** & Low \\
\hline Chaos & Very Low & Very Low & Very Low Lo*** $^{* *}$ \\
\hline
\end{tabular}

* Unless otherwise stated, differences in qualitative descriptions indicate significant differences $(p<.05)$ between groups.

** Instances of the class teacher disciplining pupils were very rare however Group $C$ teachers disciplined their pupils significantly more often than Group A or Group B teachers.

*** The only significant difference on over control was that Group $C$ teachers were rated significantly higher than Group B teachers. No other differences were significant.

**** Group C classrooms were rated significantly higher on chaos than Group A or Group B classrooms.

\subsubsection{Frequency of disciplining and levels of disruptive behaviour}

Although instances of teachers disciplining pupils and pupils displaying disruptive behaviour were rare on the whole (fewer than one fifth of COS-5 observations of these three groups of Year 5 classrooms contained any instances of a teacher disciplining a pupil), this was an area where poor schools fared less well and were rated less positively. When compared to children in excellent and good schools, children in poor schools were disciplined by the class teacher significantly more often than their peers and levels of chaos were also higher in these classrooms. 
Although the difference between the number of times disruptive behaviour was recorded during the first half of each of the COS- 5 observation cycles was not statistically significant at the .05 level, it approached significance $(p<.09)$. A more revealing approach comes from looking at the maximum scores on this item. While no instances of disruptive behaviour were recorded on this measure for any of the children in good schools or those in excellent schools $(\max =0)$, there were a number of instances of disruptive behaviour recorded for children in poor schools, and sometimes this behaviour occurred during up to half of the observation time $(\max =5$, maximum score possible $=10$ ).

When compared to excellent schools, those in poor schools were only rated significantly higher (i.e. worse) on the number of times the class teacher disciplined pupils and on the level of chaos. Once again, the difference between the number of times disruptive behaviour was recorded during the first half of each of the COS-5 observation cycles, although not statistically significant at the .05 level, approached significance. No instances of disruptive behaviour were recorded for children in excellent schools.

Interestingly, the differences on the level of over control and the target child's level of disruptiveness were only significant between poor and good schools. On both measures of disruptiveness, children in were rated as more disruptive in their lessons than children in good schools. Children in excellent schools were rated as less disruptive than children in good schools on one measure but not significantly less disruptive on the second. A possible explanation for this was that the while the first measure recorded specific instances of disruptive behaviour during a ten minute interval, the second measure was a more global rating over the entire observation. It's possible that children in academically effective schools were more enthusiastic and felt freer to express their opinions and ideas and that this behaviour was perceived as disruptive.

When considering the qualitative evidence, the nature of the differences becomes clearer. Excellent schools' classrooms were consistently rated highly on how teachers managed behaviour within their classrooms. The researchers' descriptions of classroom behaviour gave a clear sense of friendly banter between the teachers and children that took place within a well-established framework for what was acceptable behaviour. The banter between children and teachers may even account for the slightly higher level of disruptiveness recorded for this group when compared to good schools. Children in these classrooms were often encouraged to actively participate in their lessons through discussion, practical activities and even, in one case, a classroom debate, however there was a clear sense of children knowing exactly how far to take things. Classroom ratings of "chaos" were consistently low - suggesting that teachers were confident in their behaviour management techniques and that children respected the classroom rules. There was a true sense of "friendly/firm control" (S04, COS-5 (3)) with a "feeling of the teacher and children jointly seeking progress. Control is by expectation and involvement in learning. The teacher is firm and friendly" (S01, Literacy IEO).

In good schools, teachers disciplined pupils only 10 times (7 of these occurred in one school, S30) and in only two observations did the teacher discipline a child twice during the same observation (all other cases of disciplining were a single instance during an observation). 
The classroom where most of the disciplining took place was a mixed Year 5/Year 6 group and the researcher noted:

The classroom climate is quite difficult. Although the teacher endeavours to maintain a positive ethos, the number of children who challenge her authority, ignore her and create restlessness (sometimes through challenging each other) is large. I note that the majority of the disengaged children are Year 6. (S30, Literacy IEO)

The difficulties in this classroom appeared to relate mostly to the Year 6, and not the Year 5, pupils and the researcher questioned whether the older children resented being taught with the younger. Apart from this one school, discipline was not a concern in these classrooms.

Poor schools classes accounted for about three quarters of the disciplinary incidences in these three groups of schools. While in most cases, teachers disciplined only once or twice during a single observation, in two observations in the same school (S79), the teacher spent half of the observation disciplining pupils and in another school, there were instances of the teacher disciplining pupils in more than half of the observations. In this particular school, the teacher generally had to discipline pupils more than once during each observation. The overall frequency of disciplining in these classrooms was significantly higher than the frequency of teachers disciplining pupils in Year 5 classrooms in schools with medium academic effectiveness and those in academically effective schools.

\subsubsection{Behaviour management strategies}

As well as the frequency of disciplining, the groups differed in the ways in which discipline was handled within the groups. In excellent and good schools, behaviour was managed to some extent by keeping pupils engaged with their work. Children in these classrooms were significantly more engaged in their learning than children in poor schools. When teachers did have to discipline pupils, this was usually (though not always) handled sensitively and sometimes even with humour. For example, in S08, the observer remarked, "Only one child disciplined gently by the teacher" (S08, COS-5 (8)). "Teacher is very relaxed and organised. No discipline problems. All on task and enthusiastic about all of the lesson" (S08, Literacy Stipek). The firm but relaxed approach is also seen in S27 where "(t)he teacher manages some "over-the-top" responses to reproduction firmly and effectively" (S27, COS-5 (5)). When an incident occurred between a child with autism and another child, the teacher quietly took them aside to sort out the difficulty (S27, Literacy IEO). Rather than letting situations get out of control, the teachers maintained calm and order without adding to the chaos or belittling the children in any way.

One strategy used by many of these teachers was to question children who had not been listening or who were misbehaving (e.g. flicking, chatting). In S31, a child at the back of the class was throwing an object into the air. Instead of addressing this directly and drawing attention to the child's poor behaviour, the teacher asked him to work out part of a maths question the class was working on and then waited until he was able to work out the correct answer. The waiting was presumably to ensure he stayed on task (S31, Numeracy Stipek). 
This was a very effective way to quickly and quietly get the child to stop misbehaving and get back on track.

The way in which discipline was handled in the Year 5 classrooms in schools with low academic effectiveness and poor quality pedagogy was very different from the way in which it was handled in other Year 5 classrooms. In Year 5 classrooms in schools with low academic effectiveness and poor quality pedagogy, the teacher often appeared to be fighting for control, shouted and used anger and humiliation to deal with inappropriate behaviour. For example:

Teacher strives for control and to make himself heard. Shouts, lengthy lectures, public castigation. (S79, Numeracy IEO)

This particular teacher seemed almost afraid of losing complete control of his class and hurried through his lesson so quickly that, at times, the children and even the researcher could not understand him.

Threats, even just for having the wrong answer, were a main part of this teacher's repertory of discipline strategies and at one point, he threatened a boy with putting his name on the board "if you shout out the answer I don't want" (S79, IEO). 'Shouting out' was a big problem in this class and when one child complained about another who had been shouting out answers, the teacher replied "I'm not interested! I'm not in the mood for you!" The situation within the classroom became so difficult that the researcher felt obliged to help some of the children who were struggling. By the end of the lesson, the impression was of a teacher and children who were out of breath and exhausted from their struggle. Once the children had left the room, the teacher confided in the researcher, "These children don't change ... The trouble is only about four of them have been through school together; the rest have joined at different times. Each one is like a spinning top, fine on their own but put together they just bounce off each other. . These children have no respect." His sense of futility was palpable.

In another school, the teacher used personal attacks to discipline children. Her response to a restless child was to tell him/her, "Right, I'm getting sick of you. Really, really sick. You haven't stopped talking since you came in the class." Another child was sent out of the class for being restless (S82 Numeracy IEO). Reponses to discipline issues in this classroom were public and punishments extreme for the level of misdemeanour. 


\subsubsection{Over control and chaos}

Levels of over control and chaos were significantly higher in poor schools than in those in schools in the other two groups. The image that emerges from poor schools is of chaotic classrooms where teachers try to overcompensate for the poor behaviour and disorganisation by applying too much control.

What does behaviour management in Year 5 classrooms in excellent schools look like?

- Follows expectations and rules (S01, COS-5 (1):4)

- The whole class and all children are on task, but control is established by involving children in their learning (S01, COS-5 (1):5)

- The teacher was very aware of all that was happening in the class. No need for any overt action to exert discipline (S01, COS-5 (2):5)

- There was no sense of over-control (S01, COS-5 (3):5)

- The teacher uses his name several times - compliments him on his eagerness to volunteer and participate. Praises for correct answers. He smiles back at her several times. Eye contact (S02, COS-5 (1):5)

- Teacher has to discipline him once. Does so in jokey manner by saying all he will remember is the colour of the carpet (S02, COS-5 (5):5)

- Calm, quiet and firm discipline - mainly by expectation (S03, COS-5 (6):5)

- No disciplinary incidents, clear expectations. Children on task (S04, COS-5 (3):5)

- No chaos/poor discipline etc. Occasional quiet reminder of rules. All well organised (S04, COS-5 (8):5)

- No disruption. No discipline needed (S05, COS-5 (7):5)

- You can hear a pin drop in this class as children work out factors of 42 - and such a low buzz of enthusiasm (S07, COS-5 (7):5)

- The teacher has a 'strong' presence and maintains a high profile throughout . . . Feeling of teacher and children jointly seeking progress. Control by expectation, involvement in learning. Teacher is firm and friendly (S01, Literacy IEO:3)

- In whole session, no disciplinary problems. Only one very minor disagreement by 3 boys which they settled amicably (S03, Literacy IEO:3)

- The teacher established firm friendly control and the children responded (S03, Numeracy IEO:4)

- The teacher maintains firm, friendly and supportive control (S04, Literacy IEO:3)

- Teacher experienced and in control. Talks at normal volume, children listen attentively. She allows lots of quiet on task discussion in pairs and with whole class - children were on task, no disruptions observed (S05, Numeracy IEO:3)

- High expectations, challenging work, appropriate and qualified praise - and no chance to slack (S07, Literacy IEO:3)

- No discipline problems. All on task and enthusiastic about all of lesson (S08, Literacy IEO:3) 
Key findings on behaviour management:

1. Although instances of teachers disciplining children were rare, teachers in poor schools disciplined students much more frequently when compared with those in other schools.

2. Target children observed in poor schools had much higher levels of general disruptive behaviour than those in the other two groups.

3. Levels of chaos, although still low, were much higher in poor schools than in the other two groups.

4. Classrooms in excellent and good schools were indistinguishable in terms of ratings on behaviour management. None of the differences between them were statistically significant.

Year 5 teachers in excellent schools excel at:

- Managing behaviour by setting high expectations and involving the children in their learning

- Handling discipline issues privately and sensitively.

\subsection{Collaborative Learning}

Of the three main types of settings seen in schools (individual, group, whole class), the impact group work has on children's learning has been the focus of much discussion. Group work implies much more than sitting children near to each other and asking them to work together; real group work requires children to work collaboratively and this includes sharing out roles, ideas and information. Each child has a specific job and the aim is for every child in the group to achieve the learning objective(s) through working together and helping each other. Children working collaboratively, either as part of a group working with a shared remit or as part of a peer tutoring scheme, can provide excellent learning opportunities. When children have to consult each other, share knowledge and understanding and analyse and improve each other's ideas, or where one child teaches another, there is a greater likelihood of developing deep, long-lasting understanding.

\subsubsection{Time spent working in groups}

The classroom observation instruments provided information about how often children worked in groups but less detail about the specific purpose of the group work. The COS-5 required researchers to record the time the target child spent working individually, in groups and in a whole class setting, as well as the time s/he spent on collaborative work (see Table 3.6a). Because this instrument often focussed on the start of lessons, and group work is more likely to take place later on in the lesson, the incidence of collaborative group work reported was probably lower than it would have been if a whole lesson had been observed 
Table 3.6a Collaborative Learning: Time target child spends working in a group*

\begin{tabular}{|l|c|c|c|}
\hline $\begin{array}{l}\text { Characteristics of Year 5 } \\
\text { classrooms in schools with . . }\end{array}$ & $\begin{array}{c}\text { Group A: } \\
\text { Excellent schools }\end{array}$ & $\begin{array}{c}\text { Group B: } \\
\text { Good schools }\end{array}$ & $\begin{array}{c}\text { Group C: } \\
\text { Poor schools }\end{array}$ \\
\hline Target child works in a group & Very Low & Very Low & Very Low \\
\hline $\begin{array}{l}\text { Target child is involved in } \\
\text { collaborative work }\end{array}$ & Very Low & Very Low & Very Low \\
\hline
\end{tabular}

* Unless otherwise stated, differences in qualitative descriptions indicate significant differences $(p<.05)$ between groups.

Findings from the COS-5 showed that group work and collaborative learning were relatively rare occurrences (the COS-5 means were all less than 1; $\max$ score $=10$ ) and no statistically significant differences were found between the groups. The interesting finding here is that although the differences between the groups were not significant, the means showed a distinct pattern. Excellent and poor schools had slightly higher instances of group work occurring than good schools. In fact, group work was only recorded on the COS-5 for 3 good classrooms.

More instances of collaborative learning were recorded on the IEO, which records the events of an entire lesson, and group work was seen in about half of the lessons observed (see Table 3.6b). The IEO asked researchers to note whether any collaborative group work took place but did not require them to record how much time was spent working collaboratively. Any further in-depth information depended on whether the researcher included observations in the comments sections of the ratings or in the field notes of the lesson. This means that there may well have been collaborative group work taking place that was not evident in these data.

Table 3.6b Collaborative Learning: Frequency of group work observed*

\begin{tabular}{|l|l|c|c|c|}
\hline Measure & $\begin{array}{l}\text { Academic Effectiveness/ } \\
\text { Quality of Pedagogy }\end{array}$ & No & Yes & Total* $^{*}$ \\
\hline \multirow{2}{*}{$\begin{array}{l}\text { IEO: Group work seen } \\
\text { (yes or no) }\end{array}$} & High / Good (Group A) & 8 & 6 & 14 \\
\cline { 2 - 5 } & Medium (Group B) & 9 & 7 & 16 \\
\cline { 2 - 5 } & Low / Poor (Group C) & 9 & 7 & 16 \\
\hline
\end{tabular}

* Two observations per school. Totals are smaller than twice the total number of schools in each group as this measure was taken during the second year of observations only.

Teachers in excellent schools engaged their pupils in collaborative group work a little more frequently than Year 5 teachers in schools with medium or low academic effectiveness and medium or poor quality pedagogy. Although the use of groups was inconsistent and relatively infrequent compared to whole class and individual work, instances of some collaborative group work were found for many of these Year 5 classes. About a third of COS-5 observations and just under half of IEO observations included group work. Children were generally put into groups for (i) a specific, collaborative purpose (e.g. to complete a project or provide constructive feedback for each other) (ii) for ability setting or iii) so that peer tutoring could take place. 


\subsubsection{Use of groups for a specific, collaborative purpose}

When children in excellent schools spent time working collaboratively, they were often asked to act as "sounding boards" for each other or to comment on each other's work. There were also times when they worked in groups in order to solve a problem. A good illustration of groups of children working collaboratively on a task came from S03 (Numeracy IEO). The children were given the imaginary task of ordering desks for everyone at their school. The children had to work together in groups to share their understanding of measurement, develop their skill in measuring, agree a common understanding of 'middle number' and plan their work. The lesson began with sharing the objectives of the task. This was followed by group work to solve the problem and concluded with the groups sharing their findings with the entire class. The result was a lesson filled with questioning and discussion amongst the children in each group. The IEO gave a sense of busy, communicative children working together to solve a complicated problem that required every child's effort and skill.

\subsubsection{Use of groups for differentiation}

For Literacy, Year 5 teachers in excellent schools did not generally group children by ability. None of the schools grouped the children at a whole class level (for example, one class for higher ability, a second class for middle ability and a third for lower ability). Some schools were too small to do this (S01, a primary school, was made up of only 4 classes) but even larger schools that grouped children by ability for Maths did not necessarily do this for Literacy. Within a classroom, only a few of the schools grouped children by ability for Literacy and this was done by seating children of similar ability next to each other. For Numeracy, about half of the schools grouped children by ability. One school did this at the whole class level and the others grouped children by ability within the classroom. Teachers in excellent schools used differentiation more frequently than either of the other groups.

Teachers sometimes made use of these differentiated groupings to provide further teaching or extra support for a particular group of pupils. In one instance, the teacher had been explaining how to work out subtraction sums by partitioning to the whole class. Once the rest of the class began working on the differentiated worksheets she had provided, the teacher sat with the lowest ability group to provide extra teaching and support (S08, COS-5 (5)).

\subsubsection{Peer tutoring}

Although peer tutoring was not specifically recorded on the COS-5, there was some information about peer tutoring in the IEO observations. The numeracy IEO had two specific sections; 'maths discourse and communication' and 'locus of maths authority' that were potential sources of information about whether or not peer tutoring took place. The 'instructional conversations' section of the Literacy IEO was another potential source of data. Once again, the field notes of the lessons were a rich source of information about classroom practices.

It was clear from the researchers' reports that children in Group A were used to helping each other and that children often had 'response partners' with whom they worked regularly. For example, in one lesson, the teacher checked to make sure each child's response partner 
was there before setting them a task to do together: "You're going to work with response partners . . Anyone have a problem because their response partner is not here?" The children were obviously used to working in this way because they set straight to work without any confusion or chaos about what they needed to do (S01, Literacy IEO).

What does collaborative learning look like in Year 5 classrooms?

- Worked closely with peers, discussing work and approach to the problem (S01, COS$5(1): 4)$

- Took a leading role when working with peers (S01, COS-5 (2):4)

- Children were working collaboratively. The classroom was a buzz of activity (S01, COS-5 (2):5)

- Collaborative work, children involved (S01, COS-5 (3):5)

- Talking, working with peers. At times ... (the child) takes the lead in discussion (S01, COS-5 (4):4)

- A collaborative situation (S01, COS-5 (5):4)

- Cooperates well in group reading - take turns in group, no arguments. Listens well to peers read (S02, COS-5 (8):4)

- Clear evidence of good positive relationship with peers with 'response' partner and others on the table (S01, COS-5 (3):4)

- Children worked with response partners (S01, COS-5 (3):5)

- During the collaborative part, discusses well with partner" (S05, COS-5:4)

- Children participate . . . read and discuss their work (S05, COS-5 (3):6)

- Children made excellent progress in using and applying their knowledge of place value in this whole class activity. Good teaching - and opportunities to learn from contributions of others" (S07, COS-5 (2):5)

- Children are encouraged to discuss with their partner during the mental introduction to the lesson to decide on the 2 step process needed to multiply by 20 ( $\mathrm{S} 02$, Numeracy IEO:3)

Overall, instances of collaborative learning were rare in all three groups and as the number of observations was so small, significant differences were not found. When collaborative learning was observed, however, children appeared to enjoy and benefit from the experience.

Key findings on collaborative learning:

1. Instances of collaborative learning were rare.

2. Although the differences between them were not significant, collaborative learning occurred more frequently in excellent schools and in good and poor schools.

3. Group work was most often used for collaborative learning, differentiation and peer tutoring.

Year 5 teachers in excellent schools excel at:

- Providing some opportunities for collaborative work

- Encouraging children to work as 'response partners' in peer tutoring situations

- Providing opportunities for group work that required true collaboration

- Using group work as a way of devoting more time to one particular group of children 


\subsection{Personalised Teaching and Learning}

Personalised teaching and learning requires a teacher to adapt all aspects of his or her teaching to the needs of the specific individuals within a classroom group. Teachers need to recognise the individual needs of their pupils by having a good understanding of the individual children within a class, providing teaching and learning materials suitable for a range of children, scaffolding and by differentiating the work set and outcomes expected.

The COS-5 indicators that referred specifically to personalised teaching and learning included ratings of teacher sensitivity and detachment and the richness and variety of the teacher's resources. A further measure of teacher sensitivity, social support for learning, was included on the IEO. Qualitative comments on both the COS-5 and IEO, as well as the researchers' field notes of the lessons, offered more detailed descriptions about the degree and ways in which teachers attempted to personalise their pupils' learning.

Table 3.7a Personalised Learning*

\begin{tabular}{|l|c|c|c|}
\hline $\begin{array}{l}\text { Characteristics of Year 5 } \\
\text { classrooms in schools with . . }\end{array}$ & $\begin{array}{c}\text { Group A: } \\
\text { Excellent schools }\end{array}$ & $\begin{array}{c}\text { Group B: } \\
\text { Good schools }\end{array}$ & $\begin{array}{c}\text { Group C: } \\
\text { Poor schools }\end{array}$ \\
\hline Teacher detachment & Very Low & Very Low & Low \\
\hline Teacher sensitivity & Very High & High & Medium \\
\hline $\begin{array}{l}\text { Social support for learning in } \\
\text { Literacy }\end{array}$ & High & High & Medium \\
\hline $\begin{array}{l}\text { Richness and variety of } \\
\text { instructional material }\end{array}$ & High & Medium & Low \\
\hline
\end{tabular}

Unless otherwise stated, differences in qualitative descriptions indicate significant differences $(p<.05)$ between groups.

The three groups of Year 5 classrooms were again divided into two distinct groups, with poor schools rated as significantly worse on all elements of personalised learning. Excellent and good schools received similar ratings with excellent schools being rated significantly higher on several of the items.

Teachers in poor schools were rated as either low or medium on all items. These teachers showed greater detachment, less sensitivity to their pupils' needs and their teaching materials were not as varied or as well adapted to the specific needs of their pupils as those of other Year 5 teachers. Teachers in excellent schools were much more tuned in to the needs of their pupils. They were involved with their learning and were very sensitive to their needs. They were exceptionally good at providing teaching and learning materials that were adapted to the interests and abilities of their pupils.

\subsubsection{Teacher sensitivity and detachment}

In order to personalise the learning of the individuals within his or her class, a teacher must be sensitive to the individual needs of each of his or her pupils and be actively involved with their learning. Excellent schools scored significantly higher on measures of teacher sensitivity than good and poor schools. They also scored lower on teacher detachment and higher on social support for student learning than in poor schools. Teachers in good schools 
scored better (higher on sensitivity, lower on detachment) than teachers in poor schools on all measures.

Qualitative observations from the instruments clearly demonstrated how well teachers in excellent schools knew their students and how involved they were with their learning. Teachers seemed to know each child as an individual and to adapt their approaches and expectations according to the needs of each child. For example, "the teacher is very aware of the children's strengths and weaknesses and gives appropriate support and asks questions to suit those she chooses to answer, often naming them rather than choosing volunteers" (S07, COS-5 (2)). When dealing with a very anxious, off-task boy, the Year 5 teacher in S08 sat opposite him, maintained eye contact and re-explained how to complete the Maths task she had set. The technique appeared successful and by the end of the teacher's intervention, the boy had relaxed enough to smile at the teacher (COS-5 (5)).

Poor schools, by contrast, were generally rated as around the median on teacher detachment (although two schools received very low scores on this) and below the midpoint on teacher sensitivity. This indicates that teachers generally maintained a distance between themselves and their pupils and suggest they were either not aware of, or did not react to, the differing needs of the individuals within their classrooms.

\subsubsection{Variety and richness of teaching resources.}

The three groups of schools were clearly different on this measure. Excellent schools had better resources than those in good schools and the teachers of both of these groups had resources that were significantly better than those in poor schools.

The teaching resources of teachers in excellent schools were often extremely flexible and adaptable. For example, in S07, a Maths game involving children calling out times table questions to each other was used to help children learn to quickly recall their times tables. Because of the way the game was designed, the caller had to know the answer before asking the question and so the level of question could vary according to the complete ability range of children within the class (S07, Numeracy IEO).

Without exception, poor schools received ratings below the median on the variety and richness of the teaching resources used during lesson observations. There were many references to work sheets which are not necessarily a poor resource, but were perceived by the researcher as a time-filling activity rather than a learning experience.

\subsubsection{Differentiation}

Differentiation can be achieved either by using differentiated tasks and/or by ability setting. Evidence from the previous section indicates that the more academically effective the school, the more likely the Year 5 teachers were to use carefully differentiated learning materials. For example, in S08, the teacher wanted children to have a deep understanding of a poem she had read with them. She began by asking the children to visualise and draw a character from the poem, a task all children could complete together. For the next part of the lesson, the children could choose which of two tasks of different levels of difficulty they wanted to attempt. They could either write a continuation of the poem that kept to the style 
of the original poem or, for those who needed an easier task, they could write a letter to the postman asking for his help (the children had to pretend they were locked in a house and needed to escape). The researcher noted that some of the lower ability children in the class chose to attempt the more difficult task, even when the easier task was suggested.

\subsubsection{Scaffolding}

There were no direct measures of scaffolding however analysis of the qualitative observations suggests that scaffolding was far more likely to take place in schools with within excellent and good schools. For example, children in the Year 5 class in S32 were learning how to write persuasive letters to support a cause. The teacher provided both a check-list of qualities the children should look for in their own writing as well as a writing frame that clearly showed the children how their letters should be set out. The level of scaffolding may not have been necessary for the more able children however by providing such supportive resources, the teacher was able to ensure that all children, whatever their ability, would be able to attempt the task.

What does personalised teaching and learning look like in classrooms in excellent schools?

- Children were involved in their learning and enjoying the experience. Indicating teacher sensitivity and understanding of children's needs (S01, COS-5 (3):5)

- Clearly knows children well. Gives sensitive support and realistic feedback as she moves round the room (S06, COS-5 (1):5)

- Teacher is very aware of children's strengths and weaknesses and gives appropriate support and asks questions to suit those she chooses to answer, often naming them rather than choosing volunteers (S07, COS-5 (2):5)

- Knows children well. Directs some questions - and frames some for particular children (S07, COS-5 (7):5)

- Sure of her understanding of the children, their levels and needs. Also in her ability to extend appropriately (S01, Literacy IEO:3)

- Teacher encourages all to contribute ideas throughout. Children listen well to each other. Teacher uses children's names to get them to contribute (S02, Literacy IEO:3)

- Teacher says to all about boy at front 'Very good boy spending his time reading whilst he's waiting.' This is one of very shy boys, less confident. She boosts his confidence here (S05, Literacy IEO running record:1)

In summary, the individual needs of the Year 5 children in excellent and good schools were met through their teachers' friendly approach, high expectations and appropriately challenging and differentiated tasks. Excellent schools excelled at providing a rich variety of teaching and learning resources and were particularly tuned in to the needs of their individual students. Teachers in poor schools provided teaching and learning resources that were less varied and engaging and less likely to be appropriately differentiated, were less sensitive to their pupils' individual needs and more detached from their learning experiences. 
Key findings on personalised teaching and learning:

1. The quality and variety of teaching resources provided by the teacher was better in excellent and good schools than in poor schools.

2. Teachers in excellent and good schools were more involved with and aware of the individual needs of their pupils.

3. Teachers in excellent and good schools offered their pupils more social support with their learning.

Year 5 teachers in excellent schools excel at:

- Providing a good variety of high quality teaching resources

- Understanding and responding sensitively to the needs of their pupils.

\subsection{Making Links Explicit}

The COS-5 provided limited information about the cross and extra curricular links, as well as the links between previous and future learning, that teachers made explicit for their pupils. The classroom codes had several sections where references to connections to other topics could be recorded in the qualitative observations (in particular, richness and variety of instructional methods and teacher sensitivity). The IEO specifically required researchers to rate and record observations on 'cross-disciplinary connections' and 'linkage to life beyond the classroom'. In addition to these specific items, qualitative observations recorded under 'social support for student learning' on the general instruction scales, 'basic skill development in the context of problem solving' and 'maths discourse and communication' on the Numeracy IEO, and 'purposeful development of writing skills' and 'instructional conversations' on the Literacy IEO were further sources of information. Finally, the field notes of the lessons once again provided richer detail about the activities and discussions teachers used to link the learning with a particular lesson to other subjects and life outside the classroom.

\section{Table 3.8a Making Links Explicit*}

\begin{tabular}{|l|c|c|c|}
\hline $\begin{array}{l}\text { Characteristics of Year 5 } \\
\text { classrooms in schools with .. . }\end{array}$ & $\begin{array}{c}\text { Group A: } \\
\text { Excellent schools }\end{array}$ & $\begin{array}{c}\text { Group B: } \\
\text { Good schools }\end{array}$ & $\begin{array}{c}\text { Group C: } \\
\text { Poor schools }\end{array}$ \\
\hline Cross-disciplinary connections & Low $^{* *}$ & Very Low & Very Low \\
\hline $\begin{array}{l}\text { Links to life beyond the } \\
\text { classroom }\end{array}$ & Low & Low & Low \\
\hline
\end{tabular}

Unless otherwise stated, differences in qualitative descriptions indicate significant differences $(p<.05)$ between groups.

** Only the difference between Groups A and B was significant.

Because the number of observations on the IEO was small, finding statistically significant differences was less likely. Despite this, the differences on both measures approached significance $(p<.055, p<.065)$ for both cross-disciplinary connections and linkage to life 
beyond the classroom. The results on both measures for good schools were not significantly different from either of the other groups however excellent schools were rated higher (differences approached significance) than poor schools. The differences are particularly interesting when the mean, minimum and maximum values are considered.

Teachers were rated, overall, as making links to life beyond the classroom better than making cross-disciplinary connections. Teachers in poor schools were rated particularly poorly at making cross-disciplinary connections. Their mean rating was just above the minimum score $(x=1.29, \min =1)$ and the highest rating any of the teachers received was 3 (max score $=5$ ). Teachers in Group $B$ were rated in between those with high and low academic effectiveness $(x=1.62)$ and the maximum score of 4 they received was also exactly in between the maximum scores of the other two groups. Finally, although excellent schools were rated the highest on this measure, and some of these teachers were given the highest rating of 5 ( $\max$ score $=5$ ), their mean score was still below the mid point of the scale (average score $=3, x=2.25$ ). Even though these teachers were observed to be better at making cross-disciplinary connections explicit for their pupils, there was still room for improvement.

Year 5 teachers in all 3 groups of schools were scored more highly at linking their pupils' learning to life beyond the classroom than at making cross-curricular links explicit. Even so, the overall rating of poor schools was below the mid point of the scale score of $3(x=2.21)$ and once again, none received the highest score of 5 . Teachers in food schools were rated in between excellent schools and poor schools, although they were closer to excellent schools. Some of the teachers in good schools, just like some in excellent schools received the highest possible rating of 5 . Finally, teachers in excellent schools were rated as slightly above the mid point of the scale $(x=3.44)$ in their ability to link learning in the classroom to life beyond it.

In classrooms in excellent schools, teachers made cross-subject and extracurricular links explicit for their pupils. They specifically pointed out the links between what children were doing in a particular lesson and what they were learning in other subjects. They also helped their pupils to see the connections between what they learn in school and their lives and the world outside of school. Teachers in these schools made the links between academic subjects and between academic subjects and life beyond the classroom clear by pointing them out as part of their teaching (e.g. suggesting the children might enjoy re-reading the stories they are writing now later on in their lives) or by using practical activities that linked a lesson objective to the outside world.

One lesson in particular, recorded using the IEO, exemplified a way of connecting a large number of subjects together (English, Geography, Science and Personal/Social/Health Education (a road safety aspect)) and then connecting them with the children's lives both in and outside the school (all within an hour and a half). At the start of the lesson, the children were given two tasks: to write a poem using ideas from a previous Geography lesson and to look at how people affect their environment. On a previous day, children had begun looking at the school environment and the area around it in Geography and had even gone up to the school roof to look at their city. To make the link between English and Geography even stronger, the teacher asked the children to use their Geography books to write their poems. 
The children began the lesson sitting in debating groups on the carpet brainstorming words for "environment". The teacher then wrote the keywords for the lesson up on the board and the children had a whole class session discussing the previous work they had done on the topic. At the end of this session, the children moved into a lively class debate, full of questions and astute arguments. The researcher was very impressed with the quality of the debate and how well the children were able to follow the rules. From this activity, the children watched a short film and then moved back to their tables to begin writing their poems about the school environment and the effect people have on it. The observations of this lesson gave a sense of children experiencing an enormous number of activities (especially given the short time), designed to demonstrate the links between the discrete subjects and the links with the environment around their school. In pedagogical terms it also illustrated how teachers need to consider and plan for (and be responsive to) the importance of pace and variety in learning and teaching episodes.

The IEO Literacy observation for S01 offered an interesting adaptation of a teacher using children's prior knowledge not only to build and extend their understanding of one topic but also to link a second topic so that understanding of that topic was strengthened as well. The teacher had been working on haiku poems with her mixed Year 5/6 class and she began the lesson by praising the children for their work on haikus the previous day. She then explained that she was going to set them a bigger challenge: not only did they have to write another haiku but the new haiku had to show a chronological change (e.g. growing up, months of the year). By the end of the lesson, the children had not only worked through the difficulties of incorporating sequential events into the restrictive format of the haiku but had also presented their work to the rest of the class. The researcher's notes indicated that the children were highly engaged throughout the lesson and that the teacher spent a great deal of time supporting individual children.

Sometimes, as above, the teacher used previous work the children had done as a relevant reminder to help them with a task that is the same or very similar. For example, in S02 (Literacy IEO, lesson plan, p 1) the teacher reminded the children of a character profile they had written about Dickens' Scrooge to help them write a character profile of JR Tolkien's Hobbits.

An excellent example of building on prior learning came from a S04 Literacy lesson (S04, Literacy IEO). The objective of the lesson was to write a poem about the school environment however the foundations for this lesson were laid down well before. Before the start of the Literacy lesson, the children had looked at the city from the school roof, held a class debate about the local environment and watched a related film. There was a long, steady build up to the task the children were about to undertake and the children were clear about the links between the activities. 
What does making links explicit look like in classrooms in excellent schools?

In S05, the teacher linked both a Numeracy lesson and a Literacy lesson with some work the children were doing on an opera ( $\mathrm{Dr}$ Dulcamara). The Maths lesson was especially interesting because although it was linked to the opera (a work of fiction), it was also based on "real life problems". The lesson began with the teacher questioning the children about why real-life problems are important and explaining the meaning of "cross-curricular". The children then had to come up with money problems for their classmates that were related to the opera they were studying. Children created problems about the price of "Elixir" and food, drinks and flowers for the wedding. The researcher commented that that the children were engaged and very enthusiastic throughout the lesson (S05, Numeracy IEO).

Key findings on making links explicit:

1. On the whole, Year 5 teachers did not place much emphasis on making explicit the links between subjects or between life inside and outside the classroom. Teachers were generally better at making links between learning in the classroom and life outside it than they were at making the links between subjects.

2. The teachers in poor schools were the least likely to make the links between subjects and to life outside the classroom. None of these schools achieved higher than a mid point rating on the first scale ( 3 out of a possible 5 ) or a good (4 out of a possible 5 ) on the second.

3. Teachers in excellent schools were consistently better than teachers in other groups at making links, especially links to life outside the classroom, explicit. Some teachers received the highest possible rating of very high (5 out of 5 ) on both scales.

Year 5 teachers excellent schools:

- Were more consistent at making the links to areas outside the specific lesson.

- Were better able to make the connections for cross-disciplinary links as well for links to life beyond the classroom. 


\subsection{Dialogic Teaching and Learning}

Dialogic learning and teaching refers to teachers and their pupils participating in an interactive discourse about the learning in order to extend pupil thinking and understanding. This is about much more than teachers imparting knowledge; rather it's about the dialogue and questioning that both teachers and their pupils have a stake in. Dialogic teaching can go both ways; generally the teaching objectives are aimed at increasing the pupils' understanding but through this type of discourse, sometimes it is the teacher who becomes the learner!

The COS-5 allowed numerous glimpses into the classroom to see the extent of the dialogic learning and teaching that took place. In the first part of the observation scales, both the Language and Numeracy sections broke teaching down into analysis and meaning-making activities, where dialogic teaching was likely to have taken place as opposed to basic skills activities, where it was much less likely to happen. The time the teacher and the child spent on basic skills and learning facts and the time they each spent on analysis and inference was also recorded. These gave a good indication of the proportion of time where dialogic teaching might have been used.

The second part of the COS- 5 observation (the 'classroom codes') offered further insight into the use of dialogic teaching. As well as providing numerical ratings on a number of factors, researchers recorded in more detail their qualitative observations of the learning and teaching in the classroom including the 'richness of instructional methods', 'classroom climate', 'use of instructional time', 'evaluative feedback' and 'teacher sensitivity'. All of the aforementioned provided evidence for dialogic learning and teaching.

The IEO provided further information about the use of dialogic learning and teaching. On the scoring sheets, researchers recorded both their numerical ratings and their qualitative observations of Maths 'analysis', 'depth of knowledge and pupil understanding' and 'maths discourse and communication'. The Literacy IEO allowed researchers to give numerical ratings and record qualitative observations about 'reading as meaning making', 'higher order thinking in writing', 'purposeful development of writing skills' and 'instructional conversations'. These were all areas where dialogic learning and teaching was likely to take place. Added to these, researchers provided field notes of the lesson and these proved to be rich in descriptive detail.

The overall amount of time spent on teaching and learning analysis, inference or planning was very little (scores were all low or very low) and only one of the differences between the groups was significant. Children in Group A spent significantly more time learning and performing analysis and using inference skills than children in good schools. (see Table 3.9a). 
Table 3.9a Dialogic teaching and learning: comprehension and analysis activities overall*

\begin{tabular}{|l|c|c|c|}
\hline $\begin{array}{l}\text { Characteristics of Year 5 } \\
\text { classrooms in schools with . . }\end{array}$ & $\begin{array}{c}\text { Group A: } \\
\text { Excellent schools }\end{array}$ & $\begin{array}{c}\text { Group B: } \\
\text { Good schools }\end{array}$ & $\begin{array}{c}\text { Group C: } \\
\text { Poor schools }\end{array}$ \\
\hline $\begin{array}{l}\text { Target child is learning/ } \\
\text { performing analysis or } \\
\text { inference }\end{array}$ & Low $^{* *}$ & Low $^{* *}$ & Low \\
\hline $\begin{array}{l}\text { Teacher is teaching analysis, } \\
\text { inference or planning }\end{array}$ & Low & Low & Very Low $^{* * *}$ \\
\hline
\end{tabular}

* Unless otherwise stated, differences in qualitative descriptions indicate significant differences $(p<.05)$ between groups.

** Difference is significant $(p<.05)$

*** Differences are not significant

When looking more deeply into the results for Literacy and Numeracy, once again, very few differences are found. There were no significant differences for any of the Literacy measures ('comprehension', reading as meaning making', 'higher-order thinking in writing'. For Numeracy, children in poor schools spent significantly less time than children in other year 5 classes on three of the four measures ('use of maths analysis', 'depth of knowledge and understanding' and 'locus of maths authority'). This suggests that the way in which Maths is taught is particularly important.

Differences in communication were found between the three groups of Year 5 classrooms (see Table 3.9b). Children in excellent schools spent significantly more time in instructional conversations than their peers. In addition, these children, as well as children in good schools spent more time on Maths discourse and communication. Not surprisingly, communication, and the specific type of communication, is important for dialogic teaching and learning.

Table 3.9b Dialogic teaching and learning: communication*

\begin{tabular}{|l|c|c|c|}
\hline $\begin{array}{l}\text { Characteristics of Year 5 } \\
\text { classrooms in schools with . . }\end{array}$ & $\begin{array}{c}\text { Group A: } \\
\text { Excellent schools }\end{array}$ & $\begin{array}{c}\text { Group B: } \\
\text { Good schools }\end{array}$ & $\begin{array}{c}\text { Group C: } \\
\text { Poor schools }\end{array}$ \\
\hline $\begin{array}{l}\text { Instructional conversations in } \\
\text { Literacy }\end{array}$ & Very High & High & Medium $^{* *}$ \\
\hline $\begin{array}{l}\text { Maths discourse and } \\
\text { communications }\end{array}$ & High & High & Low \\
\hline
\end{tabular}

* Unless otherwise stated, differences in qualitative descriptions indicate significant differences $(p<.05)$ between groups.

** Difference is not significant.

A significant part of dialogic learning and teaching is concept development, problem solving and analysis and well over half of all lesson observations provided evidence of these. While some of these instances were brief, about three quarters of them involved sustained episodes of dialogic teaching. This indicated that while the principal component of lessons in these classrooms was not necessarily dialogic learning and teaching, this pedagogical strategy was frequently employed. We will compare this with the teaching in classrooms in less effective schools. 
In preparing her pupils to work on some money problems (as an example of a real-life application of Maths), the School 5 teacher told the children not to worry about the answers. She explained she was more concerned with them being able to identify the operations and, by implication, the strategies required to solve the problems that she was about to give the children than calculating the correct answers. At one point in the lesson, she asked the children to write down the operations required to solve a problem and told the children they were not allowed to write down the answer. Some of the children found this very hard and the teacher reassured one child by saying "You look quite hesitant. Don't worry about the answers; you are very good at Maths. Write down the operations only." This teacher went beyond offering the children in her class the opportunity to focus on strategies rather than answers by insisting that they did not work out the answers. The children were encouraged to discuss their solutions with each other and there was a great deal of sharing of ideas and strategies between the children (S05, Numeracy IEO).

In S08, 5 of the 8 COS-5 observations were rated very highly on concept development/problem solving/analysis/inference dialogic learning and teaching. The researcher commented that the children were "not stifled" (COS-5, cycle 4, p. 5) implying that children felt free to ask questions, request feedback and to discuss their work with each other and with the teacher. In this particular lesson, although the children were working on basic maths skills (number bonds to 100), the teacher encouraged them to work in small groups and discuss their work and their strategies with each other. The researcher noted that the teacher encouraged two boys with SEN to participate in the whole class activity and used questioning and humour to keep the entire class engaged in the lesson.

The S08 teacher also spent a great deal of time during both the Numeracy and Literacy IEO observations questioning the children in her class. During the Numeracy data handling lesson, she frequently asked the children to explain concepts (What is the mode? Explain what the data mean. How will we deal with all these data?) and then used their answers to further clarify their meaning (School 8, Numeracy IEO). During the Literacy lesson about a poem, she stopped often to question pupils about the inferences that could be drawn from the poem and whether or not they could justify their interpretation of one of the characters in the poem from what was actually written.

What does effective dialogic teaching and learning look like in Year 5 classrooms?

In preparing her pupils to work on some money problems (as an example of a real-life application of Maths), one teacher told the children not to worry about the answers. She explained she was more concerned with them being able to identify the operations and, by implication, the strategies required to solve the problems than she was about the children calculating the correct answers. At one point in the lesson, she asked the children to write down the operations required to solve a problem and told the children they were not allowed to write down the answer. Some of the children found this very hard and the teacher reassured one child by saying "You look quite hesitant. Don't worry about the answers; you are very good at Maths. Write down the operations only." This teacher went beyond offering the children in her class the opportunity to focus on strategies rather than answers by insisting that they did not work out the answers. The children were encouraged to discuss their solutions and there was a great deal of sharing of ideas and strategies. 
Key findings on dialogic teaching and learning:

1. Children in excellent schools spent much more time learning and carrying out analysis than their peers in good and poor schools.

2. There was a clear distinction in the approach to maths between poor schools and the approaches observed in excellent and good schools. In excellent schools teachers encouraged discussion, analysis and depth of understanding of mathematical concepts and shared the responsibility for learning with the children.

3. In literacy, teachers in excellent schools supported and promoted discussion for deeper understanding whereas this strategy was rarely seen being used by teachers in poor schools.

Year 5 teachers in excellent schools excel at:

- Providing opportunities for children to learn and practise analysis.

- Encouraging discussion, analysis and depth of understanding in Maths

- Sharing the locus of Maths authority with their pupils

- Supporting and promoting discussion for deeper understanding in Literacy 


\subsection{Assessment for Learning (AfL)}

Assessment for learning not only gives a child an indication of how well he or she is currently performing but also provides detailed guidance on how to improve. This can be done by the teacher feeding back to the entire class (for example, as part of the plenary), to groups of children or to individual children. This type of assessment can be delivered immediately by the teacher or the child's peers or, later on, as part of marking the child's work. It can be during, on, or after the task.

The COS-5 provided two indicators for assessment for learning. The first was the rating on evaluative feedback and the second was the researcher's rating of whether children could reflect on their learning through review. The IEO offered more opportunities for researchers to comment on the type of feedback and questioning teachers used in the classroom. Researchers recorded whether or not a plenary took place. This provides an important opportunity within a lesson for teachers to give feedback and to give children opportunities to feedback on each other's work. In addition to this, the scoring sheets provided further opportunities to record instances of AfL. The Numeracy IEO asked researchers to rate and record observations on 'maths discourse and communication' and 'locus of maths authority'. These are both areas where assessment, questioning and feedback from teachers and/or peers might take place. 'Instructional conversations', on the literacy IEO, also required ratings and qualitative observations and again, this was an area where assessment, questioning and feedback could take place. Finally, the field notes of the lessons provided more detail about assessment, questioning and feedback during the lesson.

Table 3.10a Assessment for learning*

\begin{tabular}{|l|c|c|c|}
\hline $\begin{array}{l}\text { Characteristics of Year 5 } \\
\text { classrooms in schools with ... }\end{array}$ & $\begin{array}{c}\text { Group A: } \\
\text { Excellent schools }\end{array}$ & $\begin{array}{c}\text { Group B: } \\
\text { Good schools }\end{array}$ & $\begin{array}{c}\text { Group C: } \\
\text { Poor schools }\end{array}$ \\
\hline Evaluative feedback & Medium & Medium & Low \\
\hline $\begin{array}{l}\text { Children can reflect on } \\
\text { learning through review }\end{array}$ & Medium $^{* *}$ & Low $^{* *}$ & Medium $^{* *}$ \\
\hline
\end{tabular}

Unless otherwise stated, differences in qualitative descriptions indicate significant differences $(p<.05)$ between groups.

** Because the scale was so small (1-3), it was difficult to reflect significant differences in the qualitative terms as they related to the means. Group C was not significantly different from Group B but was rated significantly lower than Group A.

Teachers in excellent and good schools provided more evaluative feedback than those in poor schools. In addition teachers in excellent schools provided more opportunities for their pupils to reflect on their learning through review than teachers in both good schools and poor schools. Good and poor schools did not differ in the extent to which they provided opportunities for pupils to reflect on their learning through review.

What does effective assessment for learning look like in classrooms in excellent schools?

- Feedback at the individual level and also at class level; there was depth.

- The teacher was involved with children throughout - as a whole class or with groups or individuals. Responded accordingly . . . every indication of good quality feedback. 
- Uses children's writing to model and feedback. Uses children's paragraph structures as basis for work.

- The teacher uses children's answers to model correct answers.

- Gives individual feedback to most children as she moves round.

- Children are encouraged to evaluate their own handwriting. The class teacher also gives feedback as she goes round the class discussing handwriting.

Key findings on assessment for learning:

1. Good and excellent schools provided much more evaluative feedback to their pupils than poor schools.

2. The only Year 5 teachers to receive the highest possible rating on providing evaluative feedback to their pupils were those in excellent schools.

3. Children in excellent schools were also in the only group rated as having sufficient opportunities to reflect on their learning.

The key differences between Year 5 classes in schools with medium and high academic effectiveness were:

1. Although teachers of both groups were rated much higher than Year 5 teachers in schools with low academic effectiveness on providing evaluative feedback to their pupils, Year 5 teachers in schools with high academic effectiveness were the only ones to receive the highest possible rating.

2. Year 5 teachers in highly academically effective schools provided more opportunities for the children in their classes to reflect on their learning.

Year 5 teachers in excellent schools excel at:

- Ensuring that assessment for learning was part of lessons

- Providing sufficient opportunities for children to reflect on their learning 


\subsection{Plenary}

Linked to both dialogic teaching and learning as well as to AfL, an appropriate plenary is considered an integral and valuable part of most lessons. In the quantitative data researchers only recorded plenaries in 69 schools. In the quantitative analysis the observed quality of teaching was found to be significantly higher in lessons where a teacher used a plenary in both literacy and maths and lowest where no plenary was observed in either subject. The IEO provided detailed information about whether or not a plenary session took place as well as the length, content, focus and depth of the session. There was considerable variation in the length, aim and depth of the plenary sessions; they ranged from a few minutes where the teacher checked basic understanding to a deeper exploration of issues raised within the main lesson.

In Year 5 classrooms in excellent schools: Three quarters of the IEO lesson observations in these Year 5 classrooms contained plenaries. The main uses of the plenary in these schools were to (i) informally assess children's understanding of basic concepts and skills, (ii) to provide an opportunity for children to share their work and receive feedback and (iii) to resolve issues arising from the lesson and provide a forum for collaborative problem solving.

In Year 5 classrooms in good schools: Just over half of the lessons observed using the IEO included plenaries but many of these were rushed and only covered material at a surface level. Opportunities for sharing work and deepening understanding were rare and it was almost as if the teachers included a plenary because they knew they were supposed to but didn't have enough time either to plan or deliver the plenary properly. Most plenaries were under 5 minutes long and the longest plenary (about 15 minutes) was really just a continuation of the main lesson with children solving slightly more difficult problems. In most classrooms, the teacher checked answers with the children or questioned the children about their work - but not in depth and not with the purpose of sharing strategies or providing an opportunity for higher order thinking or insightful questions.

In Year 5 classrooms in poor schools: Plenaries were recorded for only a quarter of lessons in these schools. Where the plenary did take place, it was generally short and the main purpose was often to check answers to work completed during the lesson. Opportunities for in-depth discussion, extension or reflection did not occur as part of the plenary session in these classrooms.

In S31, however, the teacher carefully managed an excellent plenary session (about ten minutes) with children working collaboratively to improve their work. The lesson had been about poetry and the children had been working on rhyme, alliteration and onomatopoeia. At the end of the lesson, the teacher held a whole class session and asked the children to write the chorus of a class poem together. The poem had to include alliteration and the children also had to find a way to include the sound of a lorry trundling along a road. By letting the children lead, and improve on each other's suggestions, the whole class contributed to this part of the writing. The teacher then asked individual children to add in the verses they had written to create a whole class poem. The session was so effective that the children were disappointed when the session ended for break time. The plenary allowed children to 
consolidate their understanding of poetry techniques, to work collaboratively by helping each other to improve and by contributing their own work and to extend their knowledge and skills.

\subsubsection{Informal assessment of children's understanding of basic concepts and skills}

The plenary was sometimes used as an opportunity for the teacher to quickly and informally assess children's understanding of basic concepts and skills. For example, groups of children in S08 had worked together to collect data, produce a tally and then a bar chart to represent their findings. The teacher displayed a bar chart from each of the groups and then asked the children, as a whole class activity, to identify the mode. Teachers in S01 and S04 also used a short plenary session to check answers at the end of a Numeracy lesson. This suggested that the plenary was seen as a useful tool to check understanding - at least at a surface level. It also suggested that its inclusion in the lesson, even in a shortened and simplified form - was important to these teachers so that they could gain feedback on pupil understanding and consolidation of learning.

\subsubsection{Providing an opportunity for children to share their work and receive feedback}

The plenary was often used as a forum for children to share their work and this usually included at least some feedback from the teacher. In S02, for example, the teacher used a short $(5 \mathrm{~min}$ ) plenary session to provide feedback to the whole class by asking three of the children to read out the character profiles of J R Tolkien's "hobbits" they had written. She was able to offer a little feedback to the individual children about their work but more importantly, she used the plenary to address some issues arising from the level of difficulty of the task. Many of the children were finding the task very hard and so the teacher was able to reassure the children, encourage them to keep trying and to offer specific suggestions about things they could try (e.g. using more semi-colons in their writing) ( $\mathrm{S} 02$, Literacy IEO).

A similar technique was employed by the S04 teacher at the end of her Literacy lesson. The children had written poems about their school environment and although most of the children would be sharing their work with the rest of the class the following day, the teacher was able to provide some immediate, general feedback by reading out some of the children's work at the end of the lesson (S04, Literacy IEO).

A particularly striking example of using the plenary as a forum for sharing work with the specific aim of providing constructive feedback came from S01 where children were working on writing haiku poems containing a chronological sequence. The teacher spent the final ten minutes of the lesson asking children to read their haikus out to the class then offering very specific suggestions for improvement. The quality of the feedback was different in this lesson because the focus was much more on finding ways to improve the work rather than just providing praise and encouragement. This gave all of the children in the class an opportunity to reflect critically on their own and each other's work, to consider what worked well and provided clear suggestions about how the haikus could be improved. This teacher exemplifies how a classroom 'community of learners' culture engages learning (S01, Literacy IEO). 
11.1.3 Resolving issues arising from the lesson and provide a forum for collaborative problem solving

The plenary session was also used for resolving issues arising from the lesson and providing a forum for collaborative problem solving. The S03 teacher asked the children to sort out an imaginary desk order for their class. The children had to consider a number of factors especially the dimensions of the desks. The children's measuring yielded a variety of results and the teacher used the plenary session to help resolve this difficulty by turning to a discussion on "averages" and "middle numbers". The children shared their results with each other and then discussed the various options for finding the most representative measurements (median, mode, mean). They were encouraged to "argue for their point of view" (S03, Numeracy IEO).

Looking at the use of plenaries across all of the Year 5 classrooms, there were few instances where the plenary - often not even present - was more than a quick recap of some of the main teaching points. Teachers tended to use the plenary to check answers with the children or to question them about their work - but the questioning was not open or in-depth and was not with the purpose of sharing strategies or providing an opportunity for higher order thinking or insightful questions.

What does a good plenary look like in Year 5 classrooms?

Some teachers were able to use the plenary to encourage in-depth thinking, sharing of work and strategies and to provide opportunities for extension. At the end of one poetry lesson introducing alliteration and onomatopoeia, the teacher asked the children to write the chorus of a class poem together. The poem had to include alliteration and the children also had to find a way to include the sound of a lorry trundling along a road. By letting the children lead, and improve on each other's suggestions, the whole class contributed to this part of the writing. The teacher then asked individual children to add in the verses they had written to create a whole class poem. The session was so effective that the children were disappointed when the lesson ended for break time. The plenary allowed children to consolidate their understanding of poetry techniques, to work collaboratively by helping each other to improve and by contributing their own work and to extend their knowledge and skills.

Key findings on the use of plenary sessions

1. Teachers in poor schools rarely used plenary sessions and when they did, they were usually short and used to check work done in lessons.

2. Teachers in excellent and good schools used plenary sessions more often

3. Teachers in poor schools used the plenary to provide extension, to deepen children's understand and to allow opportunities for reflection and feedback.

Year 5 teacher in excellent schools excel at:

- Ensuring plenary sessions take place

- Using the plenary session for extension, and for providing opportunities for children to deepen their understanding, share and reflect on their work and receive feedback 


\section{Key Findings}

There were significant differences in the strategies used by teachers in excellent schools and those teachers in poor schools. Where differences were found, the strategies used by good schools generally resembled those of teachers in excellent schools more closely than they resembled those in poor schools.

\section{Year 5 Teachers in excellent and good schools:}

- Organised their lessons, materials and teaching time well. Lessons were well-planned, time was used productively and children were more self-reliant. Teachers in excellent schools were especially good at providing well-organised and fit-for-purpose resources, making productive use of their teaching and learning time and their pupils were more self-reliant.

- Shared their learning objectives. These teachers ensured that the concepts and ideas for each lesson were clear to all children. Teachers in excellent schools were exceptionally good at ensuring the learning intentions of their lessons and activities were clear to all pupils.

- Set homework directly linked to their learning objectives. Although there was not a great deal of evidence regarding the setting and monitoring of homework and its purpose, Year 5 teachers in excellent and good schools appeared to directly link the homework they set with what the children were learning in class.

- Had positive classroom climates. These classrooms were happy places where everyone (adults and children) demonstrated mutual respect and a common purpose. Teachers in excellent schools excelled at providing positive classroom climates, being sensitive to the needs of the individual children in their classes and had excellent relationships with their pupils. Children in these classes demonstrated high levels of liking and respect of their fellow pupils.

- Spent less time disciplining their pupils. Children in these two groups had lower levels of overall disruptiveness.

- Rarely used collaborative learning. In fact, children in good schools spent the least amount of time working in collaborative settings.

- Personalised their pupils' teaching and learning. They were sensitive to their pupils' needs and provided resources to suit. Their levels of detachment were low.

- Rarely made extra and cross-curricular links explicit although teachers in excellent schools were significantly better at pointing out cross-disciplinary connections.

- Used some dialogic teaching and learning, especially in Maths. Maths discourse and the depth of the children's Numeracy knowledge and understanding were rated higher in these classrooms and the locus of Maths authority was shared with the children. 
- Used assessment for learning. Teachers in both these groups schools provided evaluative feedback to their pupils. In addition, those in excellent schools ensured their pupils had opportunities to reflect on their learning through review.

- Used plenaries more often. 


\section{Section 4 Discussion}

Of the 82 schools with Year 5 classrooms for which we have observational data analysed for this study we found them to be generally well-organised, happy places to be. Teachers' objectives were usually clear, children followed well-established class routines and resources were well-organised and appropriate for both the children and the learning objectives. Classroom climate was positive and supportive and behaviour management was handled sensitively and often through expectation. Opportunities for collaborative work, dialogic teaching and learning and assessment for learning (AfL) were rare.

\section{Organisation}

Year 5 teachers in academically effective schools with good quality pedagogy (excellent schools) organised their lessons, materials and teaching time well. Lessons were wellplanned, time was used productively and children were more self-reliant. Teachers in these schools were especially good at providing well-organised and fit-for-purpose resources, making productive use of their teaching and learning time and their pupils were more selfreliant. The quick pace in these classrooms left no time for "slacking" and there was a sense of urgency about the learning taking place.

The difference in the level of children's self-reliance is interesting. A possible explanation for it is that if behaviour is generally better in academically effective schools, then teachers are more likely to encourage greater independence in their pupils and pupils are more likely to behave within the limits of the classroom rules. If pupil behaviour is more difficult to manage, teachers may limit the degree to which pupils are allowed to take control. It's equally possible that children in these schools may be more self-reliant because their classrooms and resources are better organised. If they always knew what to do and where to find their resources, then they would be more able to function autonomously.

\section{Shared objectives}

Year 5 teachers in the schools with the highest ratings of academic effectiveness and pedagogical quality (excellent schools) shared their learning objectives with their classes. These teachers were exceptionally good at ensuring that the learning intentions of their lessons and activities and their associated concepts and ideas, were clear to all pupils. Excellent and good schools (medium effectiveness and medium quality pedagogy) teachers were also rated very highly on ensuring that concepts and ideas were clear to all children. They were significantly better at this than poor schools (low effectiveness and low quality pedagogy) teachers.

\section{Homework}

Homework set by Year 5 teachers in excellent schools seemed to serve two purposes. First, it freed up class time by allowing the learning of some basic skills (e.g. spellings, times tables) to take place at home. This, in turn, may have allowed teachers to use class time more productively. Secondly, homework set by teachers in excellent and good schools was more likely to be directly linked to children's in-class learning objectives. This could provide opportunities for deeper understanding and extension work. 


\section{Classroom climate}

Positive classroom climate seems to be a key part of classrooms in excellent schools schools. These classrooms were happy places where everyone (adults and children) demonstrated mutual respect and a common purpose. Excellent schools teachers excelled at creating warm, supportive environments where children felt safe enough to take risks with their learning. These teachers were especially sensitive to the needs of the individual children in their classes and had excellent relationships with their pupils. Children in these classes demonstrated high levels of liking and respect of their fellow pupils.

Trust, between teachers and children and between the children themselves may be partly responsible for the link between positive classroom climate, academic effectiveness and quality of pedagogy. If children don't have to worry about the reactions of their classmates or teachers to their mistakes, then they might be more willing to tackle new learning challenges. Classroom climate might also interact in a positive way with the use of collaborative work, dialogic teaching and learning and with the effectiveness of peer assessment. Collaborative work and dialogic teaching and learning are more likely to be successful in classrooms where children get on well. Children working in classrooms with a positive climate will already have good models of cooperative behaviour and more practice at working with and supporting each other and so should be able to make better use of opportunities to learn through collaborating and through dialogue.

\section{Behaviour Management}

Teachers in excellent schools managed pupil's behaviour through engaging their pupils in their learning and by sensitively, and often privately, dealing with breaches of classroom rules. They sometimes used humour as part of this technique. Levels of pupil disruption and chaos were lower in classrooms in excellent and good schools.

\section{Collaborative Learning}

Collaborative learning played a surprisingly small role in children's learning experiences. Children in good schools spent the least amount of time working in groups and children in excellent schools spent the most. Excellent schools children sometimes worked together to provide feedback for each other or to work collaboratively on a complex problem. Excellent schools teachers did not generally use groups to set children for Literacy but about half did for Numeracy. Teachers in these classrooms sometimes used these opportunities to work with a specific group of children. Excellent schools children were also used to supporting each other's learning through the use of peer tutoring and response partners.

As excellent schools classrooms have extremely positive classroom climates and few behaviour management issued, it makes sense that these are also the classrooms where the most collaborative learning takes place. Children in these classes are more self-reliant and so are able to work well together and take responsibility for not only their own learning but for their peers' as well.

\section{Personalised Teaching and Learning}

Group A teachers provided excellent teaching resources and were the teachers who were the most sensitive to the needs of their pupils. Teachers in excellent and good schools were 
less detached, more involved with and aware of the individual needs of their pupils and better able to meet those needs. They provided more social support for their pupils' learning than teachers in poor schools.

Once again, it's entirely possible that there is an interaction between the better classroom climate, pupil behaviour more collaborative approach to learning and the higher ratings teachers received on personalised teaching and learning in good and excellent schools. These teachers make a point of getting to know their students and trying to understand their needs. With the positive relationships within the classroom and this 'insider' knowledge, teachers in good and excellent schools are in a much better position to adapt their teaching to the specific interests and needs of their students.

\section{Making Links Explicit}

Teachers in all groups provided disappointingly few examples of linking their teaching with other areas of the curriculum or with the world outside the classroom. This is not particularly surprising for teachers in poor schools as they tend to be more detached from their pupils and less sensitive to their needs. This means that they would be more likely to miss opportunities to link what the children are learning with their outside interests because they might simply be unaware of these interests.

Once again, however, it was the Group A teachers who demonstrated how a good understanding of your pupils can improve their learning experiences. Although still not scoring particularly high on this item, they were rated as significantly better at making crossdisciplinary connections explicit than teachers in poor schools. With their better understanding of their pupils' interests, it should be easier for them to see these links and therefore, to explain them to their pupils.

\section{Dialogic Teaching and Learning}

Dialogic teaching and learning is clearly an area where some is much better than none. Although the overall results were a little disappointing because of the small number of recorded instances of dialogic teaching and learning, once again, where there are differences, Excellent schools teachers demonstrated the importance of this strategy as they come out on top. These teachers seem to have a talent for engaging their pupils at all levels. They arrange their classrooms, routines and resources so that pupils can be selfreliant - a necessary foundation for children taking ownership of their own learning.

Teachers in excellent schools are extremely sensitive to their pupils' needs, they model and encourage (perhaps even insist on) good relationships and, at least occasionally, provide a collaborative working environment. By laying down all of these foundations, their classroom environments are perfect for dialogic teaching and learning to take place. Children in these classrooms can confidently engage in dialogue with each other and with the teacher because they know their opinions and contributions will be respected and valued. They can attempt new learning challenges more easily because of the supportive environment in which they work.

An especially remarkable finding was that excellent and good schools teachers were much more likely to use dialogic teaching and learning in Maths. They were rated higher than 
teachers in poor schools on their use of Maths discourse and the depth of the children's Numeracy knowledge. These teachers shared the locus of Maths authority with their pupils. This finding is particularly worthy of note as Numeracy is a subject where depth of understanding is important for children to make good progress but is also a subject where pupils are least likely to be taught in this way. It's possible this difference exists because teachers in these classrooms are secure in their knowledge and understanding of Maths and therefore, can teach deep understanding as well as basic skills.

Dialogic teaching and learning will really only succeed in an environment where everyone feels safe enough to take risks, and teachers in excellent schools make sure that their classrooms are safe and supportive.

\section{Assessment for learning}

Assessment for learning seems to be part of the ongoing dialogue between teachers and pupils in excellent schools and, to a lesser extent, good schools. Teachers in excellent and good schools provided much more evaluative feedback and opportunities for their pupils to reflect on their learning than teachers in poor schools. Group 5 teachers excelled at this and were the only teachers to receive the highest possible rating on providing evaluative feedback to their pupils. This strategy is an extension of the dialogic teaching and learning that takes places within these classrooms and may well be partly due to the positive teacherchild relationships. Appropriate and encouraging feedback is another important strategy used particularly skilfully by teachers in excellent and good schools.

\section{Plenary}

The use of a plenary is another key strategy used by Year 5 teachers in academically effective schools with good quality pedagogy. Teachers in excellent schools were twice as likely to include a plenary in their lesson than teachers in poor schools. These plenaries were more than just a short session at the end of the lesson to check answers; offered children the opportunity for extension and deeper thinking about their learning. Providing opportunities for children to reflect on and consolidate their learning, deepen their understanding and extend their thinking are important elements of excellent teaching. 
What differentiates excellent practice from good practice?

Teachers in excellent schools (academically effective with good pedagogy):

- Have excellent organisational skills. They share clear learning objectives with the children in their classes and ensure that all pupils understand these objectives and their associated concepts. Their resources are extremely well organised and fit for purpose and their classroom routines are well-established, smooth and followed by all. Children in these classrooms know what they have to do, what to do if they need help and have more responsibility for managing their time and resources.

- Establish a positive classroom climate. In these classrooms, relationships between children and between adults and children are characterised by a true sense of liking and mutual respect and are often described as happy places with a "buzz" of productive learning activity. Children in these classrooms are less disruptive; behaviour management is handled sensitively and often through expectation and teachers rarely have to discipline children. Teacher sensitivity is high and teacher detachment low.

- Personalise their teaching. These teachers are sensitive to the needs and interests of their pupils and provide a variety of resources to suit the different needs of the individual children in their classes. Learning objectives are clear and shared and these teachers are more likely to make explicit the links between the learning and activities in the classroom and other subjects and the world outside the classroom. These teachers link their homework directly to what children are learning in their lessons and are more likely to take advantage of opportunities that arise during lessons to suggest learning activities that can take place out of class time.

- Use dialogic teaching and learning, especially for Numeracy. Children in their classrooms are more likely to work collaboratively, to take part in instructional conversations in Literacy, to have opportunities to receive (and probably to give) evaluative feedback (from the teacher or from their peers) and they spend more time learning and performing analysis. In Maths, these Year 5 teachers are given top marks for their use of Maths analysis, the depth of their pupils' knowledge and understanding, maths discourse and communication and their willingness to allow the children to also be the maths "authority" in the classroom. The dialogue in these classrooms was genuinely two-way; teachers were open to pupils' suggestions and corrections and used these in their teaching.

- Made more frequent and better use of the plenary. Not only were these Year 5 teachers about twice as likely to use a plenary in their lessons, they used the plenary to allow further discussion, exploration and extension, to provide opportunities for useful feedback and to consolidate and deepen understanding.

It is highly likely that good organisational skills, a positive classroom climate, personalised teaching and learning, dialogic teaching and learning and the use of a plenary session all interact with and reinforce each other. For example, dialogic teaching and learning would be impossible in a negative classroom climate. Personalising children's learning requires good organisational skills and helps both to create a positive classroom climate and to encourage discussion. 


\section{References}

Alexander, R., J. (2001). Border Crossings: Towards a comparative pedagogy. Comparative Education 37(4): 507-523.

Alexander, R. J. (2008). Pedagogy, Curriculum and Culture. Pedagogy and Practice. M. Hall. K, P and Soler, J, Open University: 3-28.

Alexander, R. J. (2010). Children, their world, their education: Final report and recommendations from the Cambridge Primary Review. London, Routledge.

Black, P., Harrison, C., Lee, C., Marshall, B., \& Wiliam, D. (2003). Assessment for Learning: Putting it into practice. Maidenhead: Open University Press.

Black, P. and D. Wiliam (1998). Assessment and classroom learning. Assessment in Education: Principals, Policy and Practice 5(1): 7-73.

Connolly, M. (2008). Letter to parents, September 2008, Arthur Terry School.

Cowan, R., Donlan, C., Newton, E. J. \& Lloyd, D. (2005) Numer skills and knowledge in children with specific language impairment. Journal of Educational Psychology 97(4), 732744.

Cowan. R, Donlan. C, Newton. E, \& Lloyd. D, (2011 In press) Number skills and knowledge in children with specific language impairment, Journal of Educational Psychology.

C4EO (2011), Centre for Excellence and Outcomes in Children and Young People's Services. Schools and Communities Research Review 4: Effective classroom strategies for closing the gap in educational achievement for children and young people living in poverty, including white working-class boys. London, Centre for Excellence and Outcomes in Children and Young People's Services (C4EO).

Darling-Hammond, L. (2002). Access to quality teaching: An analysis of inequality in California's public schools, Santa Clara Law Review.

Day, C., Kington, A., \& Sammons, P. (2008). Effective Classroom Practice: A mixed method study of influences and outcomes. Full research report, Economic and Social Research Council.

DfEE (1998). Homework: Guidelines for Primary and Secondary Schools. London, Department for Education

DfEE (1999), The National Numeracy Strategy, (London, Department for Education and Employment).

DfES (2001) The National Literacy Strategy: A Framework for Teaching. London, Department for Education and Skills 
Donlan, C., Cowan, R., Newton, E. J. \& Lloyd, D. (2007) The role of language in mathematical development: Evidence from children with specific language impairments. Cognition 103, 22-33.

Gage, N. L. (1985). Hard Gains in Soft Sciences: The Case of Pedagogy. Bloomington, Phi Delta Kappa.

Galton, M., Hargreaves, L., Comber, C., Wall, D., \& Pell, A. (1999). Inside the Primary Classroom: 20 Years On. London, Routledge.

Hallam, S. (2004). Homework: the evidence, Institute of Education, University of London.

Ireson, J., Mortimore, P., \& Hallam, S. (1999). The common strands of pedagogy and their implications. In Motrtimore $\mathrm{P}$, Understanding Pedagogy and its impact on learning. London Paul Chapman

Keiser, K. A. and L. E. Schulte (2009). Seeking the sense of community: A comparison of two Elementary Schools' Ethical Climates. The School Community Journal 45(2): 45-58.

Kesner, J. E. (2005). Gifted children's relationships with teachers. International Education Journal 6(2): 218-223.

Ko, J. \& Sammons, P (2010) Effective Teaching: A Review of Research, Report commissioned by CfBT, Oxford: Department of Education University of Oxford.

Melhuish, E. C., Sylva, K., Sammons, P., Siraj-Blatchford, I., \& Taggart, B. (2001). The Effective Provision of Pre-School Education (EPPE) Project: Technical Paper 7 Social/Behavioural and Cognitive Development at 3-4 Years in Relation to Family Background. London, DfEE / Institute of Education, University of London.

Melhuish, E., Romaniuk, H., Sammons, P., Sylva, K., Siraj-Blatchford, I., \& Taggart, B. (2006). Effective Pre-school and Primary Education 3-11 Project (EPPE 3-11): The Effectiveness of Primary Schools in England in Key Stage 2 for 2002, 2003 and 2004. Full Report. London, Institute of Education, University of London.

Melhuish, E., Phan, M., Sylva, K., Sammons, P., Siraj-Blatchford, I., \& Taggart, B. (2008). "Effects of the Home Learning Environment and Preschool Center Experience upon Literacy and Numeracy Development in Early Primary School." Journal of Social Issues 64(1): 95114.

Mortimore, P., Sammons, P., Stoll, L., Lewis, D. \& Ecob, R. (1988) School Matters: The Junior Years. Open Books Publishing Ltd. Somerset

Muijs, D. \& Reynolds D. (2011) Effective Teaching: Evidence and Practice. Sage. London

Murphy, C. E. and J. Milner (2009). Always Working an Angle:Exploration of the TeacherStudent Relationship and Engagement. Studies in Teaching 
Research Digest, Wake Forest University, Department for Education.

NICHD Early Child Care Research Network (1998). The NICHD Study of Early Child Care. Psychiatric Times 15(3): 71-72 http://www.nichd.nih.gov/od/secc/pubs.htm.

NICHD (2001). Fifth Grade School Observation Procedures Manual. NICHD Study of Early Child Care and Youth Development, NICHD Study of Early Child Care and Youth Development.

OfSTED (1995). A report from the office of Her Majesty's Chief Inspector of Schools: Homework in Primary and Secondary Schools. London, HMSO.

OfSTED (2007). The Annual Report of Her Majesty's Chief Inspector of Education, Children's Services and Skills 2006/07. London: The Stationery Office.

Pianta, R. C. (1991). The student-teacher relationship scale. Charlottesville, VA, University of Virginia.

Rose, J. (2006). Independent review of the teaching of early reading. Nottingham, DfES Publications.

Sammons, P., Sylva, K., Melhuish, E. C., Siraj-Blatchford, I., Taggart, B., \& Elliot, K. (2002). The Effective Provision of Pre-School Education (EPPE) Project: Technical Paper 8a Measuring the Impact of Pre-School on Children's Cognitive Progress over the Pre-School Period. London: DfES / Institute of Education, University of London.

Sammons, P., Sylva, K., Melhuish, E. C., Siraj-Blatchford, I., Taggart, B., \& Elliot, K. (2003). The Effective Provision of Pre-School Education (EPPE) Project: Technical Paper 8b Measuring the Impact of Pre-School on Children's Social/Behavioural Development over the Pre-School Period. London: DfES / Institute of Education, University of London.

Sammons, P., Sylva, K., Melhuish, E. C., Siraj-Blatchford, I., Taggart, B., Elliot, K., et al. (2004). The Effective Provision of Pre-School Education (EPPE) Project: Technical Paper 9 Report on age 6 assessments. London: DfES / Institute of Education, University of London.

Sammons, P., Siraj-Blatchford, I., Sylva, K., Melhuish, E., Taggart, B. and Elliot, K. (2005), 'Investigating the Effects of Pre-school Provision: Using mixed methods in the EPPE research'. International Journal of Social Research Methodology special issue on Mixed Methods in Educational Research, 8 (3), 207-224

Sammons, P., Taggart, B., Siraj-Blatchford, I., Sylva, K., Melhuish, E., \& Barreau, S, (2006a). Effective Pre-school and Primary Education 3-11 (EPPE 3-11) Summary Report: Variations in Teacher and Pupil Behaviours in Year 5 Classes. Research Report No. 817. Nottingham, DfES Publications.

Sammons, P., Taggart, B., Sylva, K., Melhuish, E., Siraj-Blatchford, I., Barreau, S., et al. (2006). Effective Pre-school and Primary Education 3-11 Project (EPPE 3-11): Variations in Teacher and Pupil Behaviours in Year 5 Classes. Full Report. London: Institute of Education, University of London. 
Sammons, P., Sylva, K., Melhuish, E., Siraj-Blatchford, I., Taggart, B., Barreau, S., Grabbe, Y (2008). Effective Pre-school and Primary Education 3-11 Project (EPPE 3-11): The Influence of School and Teaching Quality on Children's Progress in Primary School. Research Brief No. DCSF-RR028. Nottingham, DCSF Publications.

Sammons, P. and J. Ko (2008). Using Systematic Classroom Observation Schedules to Investigate Effective Teaching: Overview of Quantitative Findings. An Effective Classroom Practice (ECP) Project Report. Swindon, ESRC..

Sharp, C. (2000). Strategies for raising achievement at key stage 2: a process of educational change. TOPIC. 24.

Sharp, C., Keys, W., \& Benefield, P. (2001). Homework: a Review of Recent Research. Slough, NFER.

Siraj-Blatchford, I., Sylva, K., Muttock, S., Gilden, R., \& Bell, D. (2002). Researching Effective Pedagogy in the Early Years (REPEY): DfES Research Report 356. London, DfES, HMSO.

Siraj-Blatchford, I., Sylva, K., Taggart, B., Sammons, P., Melhuish, E. C., \& Elliot, K.. (2003). The Effective Provision of Pre-School Education (EPPE) Project: Technical Paper 10 Intensive Case Studies of Practice across the Foundation Stage, DfES Research Brief No RBX 16-03 October 2003. Nottingham, DfES Publications.

Siraj-Blatchford, I., Sammons, P., Taggart, B., Sylva, K., \& Melhuish, E (2006). Educational Research and Evidence-Based Policy: The Mixed-method Approach of the EPPE Project. Evaluation of Research in Education 19(2): 63-82.

Stipek, D. (1999). Instructional Environment Observation Scale. University of California, MacArthur Pathways through Middle Childhood Network..

Sylva, K., Melhuish, E., Sammons, P., Siraj-Blatchford, I., \& Taggart, B. (Eds.). (2010). Early Childhood Matters: Evidence from the Effective Pre-school and Primary Education project. London, Routeledge.

Watkins, C. and P. Mortimore (1999). Pedagogy: What do we know? In P. Mortimore. Understanding Pedagogy and its Impact on Learning. London, Paul Chapman Publishing.

Wells, G. (1999). Dialogic Inquiry: Towards Sociocultural Practice and Theory of Education. Cambridge, Cambridge University Press.

Whitebrook, M., Gomby, D., Ellm, D., Sakai, L., \& Kipnis, F. (2009). Teacher preparation and professional development in grades K-12 and in early care and education: Differences and similarities, and implications for research. Part I of Preparing teachers of young children: The current state of knowledge, and a blueprint for the future., CA: Center for the Study of Child Care Employment, Institute for Research on Labor and Employment, University of California at Berkeley. 
Williams, P. (2008). Independent Review of Mathematics Teaching in Early Years Settings and Primary Schools, Department for Children, School and Families (DCSF) Nottingham. 


\title{
Appendix 1 The Influence of school and teaching quality on children's progress in primary school
}

\author{
EFFECTIVE PRE-SCHOOL AND PRIMARY EDUCATION 3-11 PROJ ECT (EPPE 3-11) \\ THE INFLUENCE OF SCHOOL ANDTEACHING QUALTYY ON CHILDREN'S \\ PROGRESS IN PRIMARY SCHOOL
}

\author{
Pam Sammons $\$$, Kathy Sylva+, Edward Melhuish", Iram Siraj-Blatchford*, Brenda Taggart*, \\ Sofka Barreau* and Yvonne Grabbe* \\ \$University of Nottingham, +University of Oxford, "Birkbeck, University of London and \\ ${ }^{*}$ Institute of Education, University of London
}

\section{Introduction}

The Effective Pre-School and Primary Education 3-11 (EPPE 3-11) project investigates the impact of pre-school, primary school and the family on a range of outcomes for a national sample of $2500+$ young children in England between the ages of 3 and 11 years. This Research Brief presents analyses drawing on detailed observations of primary teachers' classroom practices in a sub-sample of 125 classes attended by EPPE 3-11 children during the time they were in Year 5 of primary school (age 10 years). It investigates the relationships between different classroom-level practices and children's progress in cognitive (Reading and Maths) and social/behavioural (Self-regulation, Hyperactivity, Pro-social and Anti-social behaviour) outcomes from Year 1 (age 6) to Year 5 (age 10). The analyses also explore associations between children's outcomes and broader measures of overall school characteristics derived from teacher questionnaires and Ofsted inspection reports.

\section{Key Findings}

- Both teachers' classroom practice and overall school-level factors make a significant difference to children's academic and social/behavioural progress during primary school after controlling for the influence of child, family and home factors and prior attainment.

- Classroom factors, particularly overall Teaching quality and Child positivity (which combines teacherchild and peer relationships and children's own self-reliance) and Parental support have an important influence on children's progress in Reading between Year 1 and Year 5. School-level factors were relatively less important for Reading. Progress in Maths, however, is relatively equally influenced by factors at classroom-level (overall Teaching quality and Quality of pedagogy) and school-level (Quality of school leadership, School communication with parents, Use of homework and school standards). This finding is in accord with EPPE 3-11 analyses for the full sample that indicate stronger school effects for children's progress in Maths (Sammons et al., 2008b).

- The influence of overall Teaching quality on Maths and Reading outcomes is stronger than the net influence of some background factors such as gender and family disadvantage (measured by eligibility for free school meals: FSM), but weaker than the influence of early years Home Learning Environment (HLE) and mothers' highest qualification level.

- The influence of primary schools upon children's social/behavioural development appears to operate more through school-level characteristics (measures of Anti-academic ethos, Use of homework and school standards and the extent of recent school Improvement since last inspection) rather than classroom-level factors. The exception is the Quality of observed pedagogy, which is beneficial both for reducing pupils' Hyperactivity, and promoting their Pro-social behaviour and Self-regulation (e.g. concentration, self-reliance). 
- The observations reveal considerable differences in teachers' and children's behaviours and classroom practices indicating significant variation in the quality of children's educational experiences during Year 5. We found that teachers can be classified into different groups in terms of overall Teaching quality, and that observed Year 5 overall Teaching quality is a significant predictor of better cognitive progress from Year 1 to Year 5 in both Reading and Maths.

- Quality of pedagogy in Year 5 (richness of instructional method, a positive climate, productive use of instructional time, the use of evaluative feedback, teacher sensitivity and lack of teacher detachment) was found to be a significant predictor of progress in Maths, but not in Reading. It was also important for children's progress in terms of reducing Hyperactivity, and promoting Prosocial behaviour and Self-regulation.

- Classroom Attention and control was related to better progress in Maths and better development in Self-regulation. Child positivity (which involved child-teacher relationships, children's cooperative skills and their self-reliance) was related to better progress in Reading.

- High levels of observed classroom Disorganisation in Year 5 (related to teachers' organisation and the behavioural climate of the classroom) were predictors of poorer progress in both Reading and Maths and increased Hyperactivity.

\section{What matters in the school}

- Children made better progress in Maths and showed improvements in Self-regulation and Prosocial behaviour where teachers reported more consistent practice across their school in the setting and marking of homework and where standards were thought to be high.

- Where teachers reported an Anti-academic ethos in their school, this predicted increased Hyperactivity and Anti-social behaviour and poorer Pro-social behavioural progress.

- Similarly, where teachers indicated that pupils had greater opportunities to organise activities for themselves and that pupils' views were listened to in their school, this predicted increased Hyperactive and Anti-social behaviour in pupils. However, where this factor was moderate (but not the greatest) pupils made better progress in Reading and Self-regulation, although the results only approached statistical significance.

- Where teachers reported the school was active in communication with parents, children made better academic progress, and showed better Self-regulation. In addition, where teachers reported strong parental support, children made better progress in Reading and Pro-social behaviour.

\section{Quality matters (Ofsted inspection measures)}

- A number of measures of school quality obtained from Ofsted inspection judgements were found to be significant predictors of children's progress and development.

- The inspection measure of overall School effectiveness was a strong predictor of better child progress in Maths and Self-regulation after control for prior development and background factors.

- Judgements of the overall Quality of school leadership also showed a modest but positive relationship with Maths progress.

- The judgement of overall school Improvement since last inspection also showed a positive association with children's Maths progress, and development in Self-regulation, Pro-social and Anti-social behaviour after control for other factors.

- $\quad$ The percentage of pupils eligible for FSM in a school was also associated with poorer children's progress in Maths, and increased Hyperactivity, Self-regulation and Anti-social behaviour (comparing those in schools below and above the mean on this factor). However the effects are somewhat weaker than those found for the Ofsted measures of overall school effectiveness and improvement. 


\section{The EPPE 3-11 Research: Background}

The original EPPE study investigated children's intellectual and social / behavioural development between the ages of 3-7 years (Sylva et al., 2004) and focussed on pre-school influences. The EPPE 3-11 study, funded by the Department for Children, Schools and Families (DCSF) has followed up the same sample of children to the end of primary school (age 11 years) and investigates both pre-school and primary school influences on children's attainment, progress and social/behavioural development. This project has now been funded to follow the same sample to the end of Key Stage 3 (age 14). The EPPE website: www.ioe.ac.uk/projects/eppe gives further details about the study and the sample.

The EPPE research adopts an educational effectiveness design using mixed methods including multilevel modelling for the analyses of child outcomes and case studies of effective practice. Earlier reports have documented the enduring impact of pre-school and the importance of early family experiences (particularly the Early years home learning environment [HLE]) on children's later attainment and social behavioural development up to age 10 in primary school (Sammons et al., 2007a; 2007b; Melhuish et al., 2008). In addition, during the pre-school phase the project explored the links between child outcomes and pre-school setting/classroom practices and processes through observations. This identified the features of pre-school experiences found to be linked with more positive developmental outcomes for young children up to age 5 years (see Sylva, 1999; Siraj-Blatchford, 2003; Sylva et al., 2006).

The analysis reported in this Research Brief investigates the way school and classroom processes influence the cognitive progress and social/behavioural development of children between the ages of 6 and 10 years old (Years 1 to 5) in a sub-sample of 1160 children (approximately $45 \%$ of the total EPPE 3-11 child sample) in 125 primary schools in England. The variation in primary teachers' classroom practice in these 125 schools has been described in
Sammons et al., 2006. The findings here build on these earlier analyses, using the same classroom observational measures. The cognitive outcome measures were collected using standardised tests ${ }^{6}$ of Reading and Maths (not national assessments) to avoid any possible influence associated with preparations for national assessments. The social / behavioural measures were derived from teacher completed pupil profiles (covering the four dimensions of Hyperactivity, Self regulation, Anti-social behaviour and Pro-social behaviour). Children's progress was measured over a four year period and thus is likely to reflect the overall influence of schools and teachers across several school years, but the observational data were confined to Year 5. This strategy enables both school and class influences on children's academic and social/behavioural progress to be explored ${ }^{7}$.

The aim is to establish whether particular features of classroom practices and school processes help to predict child outcomes after controlling for a range of background factors (child, family, HLE, prior attainment). Multilevel modelling and the use of Effect Sizes (ES) allow such comparisons to be made $^{8}$. The research focuses on measuring children's progress and development in cognitive and social / behavioural outcomes between Year 1 to Year 5.

For further details on the research and analysis used in this study, see the Research Report (Sammons 2008a).

\section{What matters in the classroom}

It was hypothesised that higher quality classroom experiences would predict better child outcomes in Year 5, taking account of children's prior attainment and social/behavioural development and background factors. School effectiveness research has

\footnotetext{
${ }^{6}$ NFER tests of Reading and Maths.

${ }^{7}$ Ideally observations would have been conducted in all years in KS 2 but this was not practical so $\mathrm{Yr} 5$ was chosen as representative KS2 experience in a school, and it was also the year when children's outcomes were assessed by EPPE.

${ }^{8}$ An effect size is a statistical measure that can be used to illustrate the strength of impact of different factors used to predict variation in children's outcomes.
} 
drawn attention to the importance of the classroom level in accounting for variations in pupil outcomes but such research has generally only tested limited measures of classroom processes and usually only examined cognitive outcomes (Teddlie \& Reynolds, 2000). The EPPE research has studied a detailed set of classroom measures obtained from observations in Year 5 and a number of different indicators of children's outcomes and therefore can explore the relative strength of different features on children's progress in a wide range of outcomes.

\section{Overall Teaching Quality}

Teachers' and children's observed behaviours were found to differ significantly across the 125 Year 5 classes (Sammons et al., 2006). The observation instruments identified significant variations in observed quality indicating that children's educational experiences in Year 5 classes differed significantly with some benefiting from higher quality experiences. The present analyses indicate that teachers could be divided into groups based on observations of their overall quality of teaching.

The classroom observation data revealed a number of underlying factors (dimensions) and these were tested to explore relationships with children's progress in a range of outcomes.

\section{Aspects of teaching}

A number of classroom processes were identified from the observational data such as Quality of pedagogy, Disorganisation, Child positivity, pupils' Positive engagement and the extent of Attention and control, as well as specific features of practice related to literacy and numeracy teaching. A measure of overall Teaching quality derived from these dimensions was constructed and teachers were grouped in terms of overall quality of practice. This measure of overall Teaching quality was a significant and moderately strong predictor of better Reading $(E S=0.37)$ and Maths progress $(E S=0.35)^{9}$.

\footnotetext{
${ }^{9}$ The strength of predictors can be reported in terms of Effect sizes (ES) these provide a summary measure of the strength of prediction of an outcome net of other predictors. An ES of 0.1 is relatively weak, one of 0.35 moderately strong, one of 0.7 strong, for example.
}

It is possible to use effect sizes (ES) to compare the strength of different factors such as overall Teaching quality with that of other background influences on children's progress for this subsample of EPPE children. For example, the influence of overall Teaching quality (Maths ES= 0.35 ; Reading $E S=0.37$ ) is smaller than the influence of early years Home Learning Environment (Maths ES $=0.37$; Reading $\mathrm{ES}=0.62$ ) and mothers' highest qualification level (having a degree versus no qualifications, Maths ES=0.63; Reading $E S=0.83$ ) and larger than the net influence of either gender (Maths $E S=0.10$ ); Reading $E S=0.05$ ) or eligibility for FSM (Maths not significant; Reading $E S=0.19$ ). Further details of background effects are shown in the full report.

Quality of pedagogy: This more detailed measure was part of the overall Teaching quality assessment and included items such as richness of instructional method, a positive classroom climate, productive use of instructional time, the use of feedback, teacher sensitivity and lack of teacher detachment. This measure was significantly related to children's progress in Maths ( $E S=0.27)$. Overall, this factor describes a classroom where teachers provide a rich learning environment where pupils are challenged in their learning and provided with specific evaluative feedback on how to improve their work. Reviews of school and teacher effectiveness research have suggested that schools vary more in their effects on Maths than on Reading (Scheerens and Bosker, 1997; Muijs and Reynolds, 2005). The current results are in accord with such conclusions showing that variations in the Quality of pedagogy in Year 5 classes are particularly important in accounting for differences in children's Maths progress.

The factor Disorganisation was related to the observed behavioural climate of the classroom and results support earlier studies indicating that fostering a calm and orderly climate is important for learning and teaching. Previously, EPPE reported moderate associations between the level of socially disadvantaged pupils in a school and the extent of Disorganisation observed in Year 5 classes (Sammons et al., 2006). Higher levels of Disorganisation were significantly associated with poorer Reading $(E S=0.21)$ and 
Maths $(E S=0.34)$ progress and also increased Hyperactivity $(E S=0.37$ ). It may be harder for teachers to maintain good order in class in schools serving higher proportions of disadvantaged children. Also poorer classroom practice may be a contributory factor in explaining the poorer outcomes of children in more disadvantaged communities. Probably both explanations play a part. Our analyses controlled for a wide range of background measures, including parents' qualifications, occupations and income. Other research (Ross \& Hutchings, 2003; Darling-Hammond, 2002) has suggested that schools in disadvantaged settings can find it harder to recruit and retain teachers and tend to be served by less experienced staff. This may play a part in the finding of a negative association between higher quality practice observed in Year 5 classes and overall level of disadvantage of pupil intake.

In addition the factor Attention and control was found to be linked to better Maths progress $(E S=0.27)$ and Self-regulation $(E S=0.36)$ while Child Positivity, (the nature of child-teacher relationships, children's co-operative skills and their self-reliance) was a predictor of better Reading progress (ES $=0.39$ ).

\section{What matters in the school}

In addition to classroom observations, the teachers of the observed classes completed a questionnaire. From this questionnaire several measures were constructed.

Use of homework and school standards Teachers' perceptions of the Use of homework and school standards (including such items as teachers set homework every week for their class, most teachers mark and return homework promptly, and whether the overall standards set for pupils at the school were perceived to be high enough) also showed a significant association with better progress in Maths with the most positive impact for the medium versus low group ( $E S=0.27)$. Higher scores here were also associated with improvements in Selfregulation $(E S=0.32)$ and Pro-social behaviour $(E S=0.33)$.

\section{Pupil agency and voice}

The factor based on teachers' perceptions of Pupil agency and voice (such as pupils organise activities for themselves and whether pupils' views are listened to and taken seriously) was associated with better Reading progress (where medium amounts showed the most positive effect, $E S=0.26$ ) and with Self-regulation ( $E S=0.27$, where higher amounts were best).

It was hypothesised that children would show positive social behaviour in schools where their views are listened to and accommodated, however, the findings suggest otherwise. Contrary to expectations, EPPE children's Hyperactive and Anti-social behaviour was significantly increased in schools where teachers reported High levels of Pupils' agency and voice ( $E S=0.30$ and $E S=0.38$ respectively). These schools may be responding to poor pupil behaviour by giving more emphasis to pupils' voice so Pupils' agency and voice may be seen as a constructive response by schools seeking to counter negative behaviour. Alternatively, some moderate amount of involvement and autonomy may be optimum, and beyond a certain point, children at this age may not respond well to high levels of autonomy because such strategies may adversely affect the disciplinary climate. Further study of Pupil Agency and voice is needed to explore these associations and their impact in more depth.

\section{Anti-academic ethos}

Where teachers reported an Anti-academic ethos amongst pupils in their school, children in the sample also showed poorer progress in Reading ( $E S=0.31)$ and Maths ( $E S=0.37$ ), increased Hyperactivity $(E S=0.36)$ and Antisocial behaviour $(E S=0.31)$ and poorer Prosocial behavioural $(E S=0.38)$ development.

\section{Parental communication and support}

Two aspects of the home-school relationship (School communication with parents and Parental support for their child's learning) were significant predictors of better child outcomes.

Teachers' perceptions of the school's communication with parents was the stronger of these two predictors (Reading $E S=0.38$, Maths $E S=0.34$ ) with children making better progress 
in schools where teachers reported good communication (such as parents being regularly informed about their child's progress / achievements and the school being good at communicating its expectations of pupils to parents). This factor also predicted better developmental progress for Self-regulation $(\mathrm{ES}=0.27)$.

Teachers' judgements of overall level of parental support for children's learning also showed a positive relationship with pupils' progress in Reading $(E S=0.28)$ and their Pro-social development $(E S=0.38)$.

\section{Quality matters (Ofsted inspection measures)}

In addition to investigating the impact of observed classroom processes, further analyses explored the predictive power of more global indicators of school quality, based on Ofsted inspectors' judgements. Several classroom process factors derived from the classroom observations were positively related to Ofsted measures of overall school effectiveness, improvement and leadership. The observed measure of overall Teaching quality was found to be higher in schools independently identified by inspectors as showing better quality in overall judgements of effectiveness, improvement and leadership (Sammons et al., 2006).

\section{School effectiveness}

School effectiveness as judged by Ofsted was found to be a strong predictor of better outcomes for children after control for prior attainment or prior social behaviour and other background factors. Attending a school judged by Ofsted as more effective made a significant difference to Maths progress $(E S=0.41)$ and for Self-regulation (ES=0.39). The results for Reading also indicated that children made more progress in schools judged to be more effective by Ofsted $(E S=0.30) \quad$ All other progress measures show effects that were in a similar direction but were not statistically significant.

\section{Improvement since last inspection}

The Improvement since last inspection indicator also showed a very similar pattern to findings on overall school effectiveness with schools that had shown most improvement being significantly associated with better progress for our sample in Maths $(E S=0.35)$, Self-regulation $(E S=0.49)$, Pro-social behaviour $(E S=0.43)$ and improvements in terms of reduced Anti-social behaviour ( $E S=0.31)$.

\section{Leadership}

The findings for the Ofsted School Leadership indicator also showed a significant positive relationship with better progress in Maths $(E S=0.32)$, and similar benefits for reduced Hyperactivity $(E S=-0.22)$ and Anti-social behaviour ( $E S=-0.23$ ), but these latter two results only approached statistical significance.

\section{Measures of social disadvantage (Free school meals - FSM)}

The level of disadvantage of the school's pupil intake ( $\%$ of pupils eligible for FSM) was related to children's cognitive and social/behavioural progress (for Maths, Hyperactivity, Selfregulation and Anti-social behaviour). A higher level of social disadvantage was a predictor of poorer progress. These analyses controlled for all other significant child, family and HLE characteristics including the individual child's family income (measured in Year 1 at age 6) and eligibility for FSM. However, the effects are somewhat weaker and less significant (ES range 0.23-0.29) than those found for the Ofsted measures of school effectiveness and improvement (ES range 0.27-0.49). Nonetheless, these findings support other research on the influence of school composition and indicate that the challenges in raising attainment are greater for schools in areas of higher disadvantage.

Elsewhere we have shown that only one of the classroom observation measures was associated with level of disadvantage (Sammons et al., 2006). Classroom Disorganisation was weakly negatively associated with overall social disadvantage of pupil intake to a school (\% pupils eligible for FSM, Correlation $r=-0.36)$.

\section{Conclusions and Implications}

Reviews of school and teacher effectiveness research have pointed to the importance of a range of school and classroom features that promote better educational outcomes for 
students, see Teddlie \& Reynolds (2000), Scheerens \& Bosker (1997) and Sammons (2007c). These include a positive school culture, good leadership, creating a positive learning environment, high expectations and good quality teaching.

The analyses of classroom observations show that it is possible to group teachers in Year 5 primary classes in terms of their overall Teaching quality across a range of observed classroom behaviour and practices. Overall Teaching quality was a significant predictor of better cognitive progress for children across the period Years 1 to 5 , and this is particularly evident in comparing the High and Low groups. In other words, children in schools where Year 5 overall Teaching quality was observed to be High do significantly better in both Reading and Maths progress than those attending schools where Year 5 overall Teaching quality was observed to be Low. However, overall Teaching quality was not associated with social/behavioural progress. It appears that the overall quality of teaching as measured by the observation instruments has a greater influence on children's academic progress, than on other social/behavioural outcomes.

With regard to other school measures, children who attended more effective and improved schools, as measured by earlier Ofsted judgements made during regular school inspections, showed longer term benefits on a range of social / behavioural outcomes as well as academic outcomes. The judgement of school leadership also showed a significant though weaker positive influence. This supports the conclusions of school effectiveness research that schools matter. Even when the powerful influences of child, family and home are controlled, going to a 'better' primary school exerts a positive net influence on children's academic progress and also on social / behavioural outcomes.

In addition, the results indicate that teachers' perceptions of a number of features of their schools (such as communication with parents, parental support, consistent emphasis on homework, pupil agency and voice, and antiacademic ethos) are also significant predictors of children's academic progress and social / behavioural progress from Year 1 to Year 5.

The findings confirm that there are significant variations in the overall observed quality of teaching in Year 5 classes and such variations are important predictors of children's progress in Reading and Maths. Specific features of teachers' practice and children's responses are also predictors of better social / behavioural outcomes. In addition, some aspects of organisation such as the school's emphasis on communication with parents and homework, and level of parental support also show a significant impact in promoting better progress. In combination with the findings on teacher quality, the results point to important features of schools and classroom processes that help to explain differences in children's outcomes and thus provide evidence on successful practice.

Taken together these results demonstrate those aspects of teachers' classroom practice and overall features of schools, including inspection judgements of school quality that have predictive validity in terms of better child outcomes at age 10. They support conclusions from previous school and teacher effectiveness studies that point to important variations between teachers and schools in their effects on pupils' progress and social/behavioural development. The quality of classroom teaching and the overall quality of the school both matter, while contextual influences and communication with parents are also significant. We conclude that initiatives that place a stronger emphasis on promoting the overall quality of teaching and creating a more orderly classroom climate are likely to improve educational outcomes for all children and may be particularly important for schools with higher proportions of disadvantaged children (because these schools are more likely to have higher levels of classroom disorder).

After taking account of other influences it is clear that the quality of classroom teaching matters, and the overall school characteristics also matter. Children in the EPPE sample who had the benefit of attending a primary school independently judged by Ofsted to be more 'effective and improved' showed both better 
academic progress and social / behavioural development. The findings also provide some independent confirmation that inspection judgements of effectiveness and improvement provide useful indicators of differences in school quality that have a measurable impact on a range of pupil outcomes. Both the quality of teaching and the overall effectiveness of the school are found to be significant predictors of better cognitive progress and social/behavioural development. Given that many studies (including EPPE 3-11) have demonstrated links between pupil disadvantage (such as low SES and family income) and significantly poorer educational outcomes, enhancing the quality of teaching and the overall effectiveness of the school are likely to be particularly important for disadvantaged groups of pupils. A major research review by Scheerens \& Bosker (1997) concluded that school effects are larger for ethnic minority and disadvantaged groups (our sample size does not allow detailed analysis of school effects for specific sub-groups here) and is supported by findings for all primary schools in England (Melhuish et al., 2006). Therefore improving the quality of teaching and overall effectiveness of the school is likely to be necessary to promote better long term educational outcomes for disadvantaged groups of pupils in particular. This conclusion is also supported by recent Ofsted findings of schools in disadvantaged areas (Ofsted, 2007). ${ }^{10}$ The EPPE 3-11 findings support the view that quality of classroom practices and overall quality of schools and their leadership make an important difference to children's academic and social/behavioural progress. The results suggest the need to reduce the variation in the quality found between class practices and school processes in order to raise overall standards and promote greater equity.

This longitudinal study is the first of its kind in England (educational effectiveness) to provide recent and robust evidence on the role of classroom influences such as teaching quality and school processes in shaping primary children's progress and development (on a wide range of outcomes) during Key Stage 2.

\footnotetext{
${ }^{10}$ See Ofsted Annual Report 2006/2007 (para. 282-284, p. 69 -70).
}

Confidence in findings is enhanced by the extent of controls for the impact of other background factors in the models used.

\section{Methodology}

The EPPE 3-11 study is the first large-scale longitudinal study to combine a wide range of data that explore the relationship between detailed measures of child, family and home learning environment (HLE) and children's progress (in both cognitive and social / behavioural outcomes) and to link this detailed information to what teachers do in the classroom and measures from inspection judgements (Ofsted). This has enabled the exploration of the predictive power of different measures in accounting for variation in children's progress across a wide range of outcomes, both academic and social behaviour.

This report describes the results of quantitative analyses based on a sub-sample of 1160 EPPE 3-11 children across Years 1 to 5 of primary education (age 6 to 10 years), representing approximately $45 \%$ of the total EPPE child sample. The research builds on the earlier analyses of children's Reading and Maths attainments and social/behavioural outcomes in Year 5 for the full EPPE 3-11 sample (see Sammons, 2007a; 2007b), by investigating relationships between children's outcomes and measures of classroom processes, collected through observation of Year 5 classes in 125 focal schools chosen from the wider EPPE 3-11 data set. The focus on progress and development across four successive years is likely to enhance the chances of identifying school influences as well as those relating to teachers' classroom practices.

Two instruments were used for classroom observations: the Classroom Observation System for Fifth Grade (COS-5, NICHD/Pianta 2001) and The Instructional Environment Observation Scale (IEO, Stipek, 1999). For detailed descriptions of these instruments see Sammons et al., (2006). Observations were conducted in Year 5 to avoid any influence associated with end of Key Stage 2 National assessments (this reduces the likelihood that 
any teaching to the test might affect results). Field work was carried out during spring and summer terms of 2004 and 2005. In addition, teachers were asked to complete a questionnaire with a $94 \%$ response rate.

Multilevel models were used to test the predictive power of different measures of classroom processes and overall quality of teaching. It should be noted, that the models of children's progress control for prior attainment (or prior social behaviour) measured in Year 1, as well as a wide range of child, family and HLE influences. The progress and developmental gains are thus measured over a four year period in primary school. The outcomes studied include Reading and Maths (measured by NFER standardised tests) and four social / behavioural measures derived from teachers' ratings of individual children (Hyperactivity, Selfregulation, Anti-social behaviour and Pro-social behaviour).

In addition global measures of overall Teaching quality were derived and tested to establish whether there were meaningful groupings of classes on the basis of overall quality.

It was hypothesised that children would make more cognitive progress and show more positive social/behavioural development in schools that had been rated more favourably on Ofsted indicators of quality in previous inspection reports, taking into account other influences. Ofsted measures were tested in the multilevel models of children's outcomes in Year 5 and the results supported the hypothesis.

\section{References}

Darling-Hammond, L. (2002), Access to quality teaching: An analysis of inequality in California's public schools: Santa Clara Law Review.

Melhuish, E., Romaniuk, H., Sammons, P., Sylva, K., Siraj-Blatchford, I. \& Taggart. (2006)

The Effective Pre-school, Primary and Education Project (EPPE 3-11): The Effectiveness of Primary Schools in England in Key Stage 2 for 2002, 2003 and 2004. Full Report London: Institute of Education, University of London http://www.ioe.ac.uk/schools/ecpe/eppe/eppe311/eppe3-11pubs.htm

Melhuish, E., Sylva, K., Sammons, P., SirajBlatchford, I., Taggart, B. and Phan, M. (2008), 'Effects of the Home Learning Environment and pre-school center experience upon literacy and numeracy development in early primary school.' Journal of Social Issues, 64, 157-188.

Muijs, D. and Reynolds, D. (2005), Effective Teaching. London: Sage.

NICHD. (2001), 'Fifth Grade School Observation Procedures Manual.' NICHD Study of Early Child Care and Youth Development: NICHD Study of Early Child Care and Youth Development. Developed by R. Pianta.

Ofsted. (2007), The Annual Report of Her Majesty's Chief Inspector of Education, Children's Services and Skills 2006/07. London: The Stationery Office.

Ross, A. and Hutchings, M. (2003), Attracting, Developing and Retaining Effective Teachers in the United Kingdom and Northern Ireland: OECD Country Background Report: Institute of Policy Studies in Education, London Metropolitan University.

Sammons, P., Taggart, B., Siraj-Blatchford, I., Sylva, K., Melhuish, E., Barreau, S. and Manni, L. (2006), Effective Pre-school and Primary Education 3-11 (EPPE 3-11) Summary Report: Variations in Teacher and Pupil Behaviours in Year 5 Classes. Research Report No. 817. Nottingham: DfES Publications.

Sammons, P., Sylva, K., Melhuish, E., SirajBlatchford, I., Taggart, B. and Barreau, S. (2007a), Effective Pre-school and Primary Education 3-11 Project (EPPE 3-11): Influences on Children's Development and Progress in Key Stage 2: Social/behavioural Outcomes in Year 5. Research Report no. DCSF-RRO07. Nottingham: DfES Publications.

Sammons, P., Sylva, K., Melhuish, E., SirajBlatchford, I., Taggart, B., Grabbe, Y. and Barreau, S. (2007b), Effective Pre-school and Primary Education 3-11 Project (EPPE 3-11) 
Summary Report: Influences on Children's Attainment and Progress in Key Stage 2: Cognitive Outcomes in Year 5. Report No. RR828. Nottingham: DfES Publications.

Sammons P (2007c) School Effectiveness and Equity: Making connections. CfBT Education Trust. Reading.

www.cfbt/evidenceforeducation.com

Sammons, P., Sylva, K., Melhuish, E., SirajBlatchford, I., Taggart, B., Barreau, S. and Grabbe, Y. (2008), The Influence of School and Teaching Quality on Children's Progress in Primary School. Report No. DCSF-RR028. Nottingham: DfES Publications.

Scheerens, J. and Bosker, R. (1997), The Foundations of Educational Effectiveness. Oxford: Pergamon.

Siraj-Blatchford, I., Sylva, K., Taggart, B., Sammons, P., Melhuish, E. C. and Elliot, K. (2003), The Effective Provision of Pre-School Education (EPPE) Project: Technical Paper 10 Intensive Case Studies of Practice across the Foundation Stage. London: DfES / Institute of Education, University of London.

Stipek, D. (1999), Instructional Environment Observation Scale. University of California: MacArthur Pathways through Middle Childhood Network.

Sylva, K., Siraj-Blatchford, I., Melhuish, E. C., Sammons, P., Taggart, B., Evans, E., Dobson, A., Jeavons, M., Lewis, K., Morahan, M. and Sadler, S. (1999), The Effective Provision of Pre-School Education (EPPE) Project: Technical Paper 6 - Characteristics of the Centres in the EPPE Sample: Observation Profiles. London: DfEE / Institute of Education, University of London.

Sylva, K., Melhuish, E. C., Sammons, P., SirajBlatchford, I. and Taggart, B. (2004), The Effective Provision of Pre-School Education (EPPE) Project: Final Report. London: DfES/ Institute of Education, University of London.

Sylva, K., Siraj-Blatchford, I., Taggart, B., Sammons, P., Melhuish, E., Elliot, K. and
Totsika, V. (2006), 'Capturing Quality in Early Childhood Through Environmental Rating Scales'. Early Childhood Research Quarterly 21 (1), 76-92.

Teddlie, C. and Reynolds, D. (2000), The International Handbook of School Effectiveness Research. London: Routledge/Falmer.

\section{Additional Information}

Copies of the full report (DCSF-RR028) - priced $£ 4.95$ - are available by writing to DCSF Publications, PO Box 5050, Sherwood Park, Annesley, Nottingham NG15 ODJ.

Cheques should be made payable to "DCSF Priced Publications".

Copies of this Research Brief (DCSF-RB028) are available free of charge from the above address (tel: 084560222 60). Research Briefs and Research Reports can also be accessed at www.dcsf.gov.uk/research/

Further information about this research can be obtained from Jessica Dunn, W606, DCSF, Moorfoot, Sheffield S1 4PQ.

Email: jessica.dunn@dfe.gsi.gov.uk

The views expressed in this report are the authors' and do not necessarily reflect those of the Department for Education. 
Summary table of relationships between different class and school predictors and children's progress in Cognitive and social/behavioural outcomes (Year 1 - Year 5)

\begin{tabular}{|c|c|c|c|c|c|c|}
\hline & Reading & Maths & Hyperactivity & Self-regulation & Pro-social & Anti-social \\
\hline \multicolumn{7}{|l|}{ COS-5 (Pianta) } \\
\hline Overall teaching quality & 0.37 & 0.35 & & & & \\
\hline Quality of pedagogy & & 0.27 & 0.28 & 0.17 & $\begin{array}{c}0.27 \\
\text { Low-Med } \\
\text { gp }\end{array}$ & \\
\hline Disorganisation & 0.21 & 0.34 & 0.37 & & & \\
\hline Child Positivity & 0.39 & & & & & \\
\hline Positive engagement & & & & & $\begin{array}{c}0.33 \\
\text { Low-Med } \\
\text { gp }\end{array}$ & \\
\hline Attention and control & & 0.27 & & 0.28 & & \\
\hline \multicolumn{7}{|l|}{ IEO (Stipek) } \\
\hline \multicolumn{7}{|l|}{ Literacy } \\
\hline \multicolumn{7}{|l|}{ Global indicator } \\
\hline Pedagogy & 0.23 & 0.45 & & & & \\
\hline \multicolumn{7}{|l|}{ Subject development } \\
\hline \multicolumn{7}{|l|}{ Learning linkages } \\
\hline \multicolumn{7}{|l|}{ Numeracy } \\
\hline \multicolumn{7}{|l|}{ Global indicator } \\
\hline Pedagogy & & 0.23 & & & & 0.28 \\
\hline Subject development & $\begin{array}{c}0.32 \\
\text { Med gp }\end{array}$ & & 0.28 & & & \\
\hline \multicolumn{7}{|l|}{ Learning linkages } \\
\hline \multicolumn{7}{|l|}{ Teacher perceptions } \\
\hline $\begin{array}{l}\text { School communication } \\
\text { with parents }\end{array}$ & 0.38 & 0.34 & & 0.27 & & \\
\hline $\begin{array}{l}\text { Parental support of their } \\
\text { child's learning }\end{array}$ & 0.28 & & & & 0.38 & \\
\hline $\begin{array}{l}\text { Use of homework and } \\
\text { school standards }\end{array}$ & & $\begin{array}{c}0.27 \\
\text { Medium grp }\end{array}$ & & 0.32 & 0.33 & \\
\hline Pupils' agency and voice & $\begin{array}{c}0.26 \\
\text { Med-high grp }\end{array}$ & & 0.30 & 0.27 & & 0.38 \\
\hline Anti-academic ethos & 0.31 & 0.37 & 0.36 & & 0.38 & 0.31 \\
\hline \multicolumn{7}{|l|}{ Ofsted judgements } \\
\hline School effectiveness & 0.30 & 0.41 & 0.27 & 0.39 & 0.37 & 0.35 \\
\hline $\begin{array}{l}\text { Improvements since last } \\
\text { inspection }\end{array}$ & 0.31 & 0.35 & 0.34 & 0.49 & 0.43 & 0.31 \\
\hline Quality of assessment & & 0.28 & & & & \\
\hline Leadership & & 0.32 & 0.22 & & & 0.23 \\
\hline \multicolumn{7}{|l|}{ Attendance } \\
\hline$\%$ of pupils eligible for FSM & & 0.23 & 0.25 & 0.25 & & 0.29 \\
\hline
\end{tabular}

${ }^{*} p<0.05$ appears in BOLD, others are approaching significance. blank cells indicate non significance 


\title{
VARIATIONS IN TEACHER AND PUPIL BEHAVIOURS IN YEAR 5 CLASSES
}

\author{
Pam Sammons $\$$, Brenda Taggart*, Iram Siraj-Blatchford*, Kathy Sylva ${ }^{+}$, Edward Melhuish" and \\ Sofka Barreau* \\ *Institute of Education, University of London, ${ }^{+}$University of Oxford, \\ ${ }^{\#}$ Birkbeck, University of London and ${ }^{\$}$ University of Nottingham
}

The EPPE 3-11 Project builds on the work of the earlier Effective Provision of Pre-School Education (EPPE) project, which was the first major longitudinal study in Europe to investigate the impact of pre-school provision on a national sample of young children, tracing their development between the ages of 3 and 7 years. EPPE 3-11 follows the same sample of 2500 plus children to age 11 years, the end of Key Stage 2 (KS2). This research brief reports the results of detailed observations of practice conducted in 125 Year 5 classes attended by EPPE children, and measures the variation in teachers' organisation and pedagogy and in pupils' responses. The brief describes patterns of association between indicators of primary school effectiveness (measured using value added approaches and national assessment results) and quality (measured by Ofsted inspection grades) and differences between Year 5 classes in observed practice and behaviour. The observations were conducted in a range of lessons with a particular emphasis on the core subjects.

\section{Key findings}

Pedagogy

- There was significant variation in both teachers' classroom practice and pupils' behaviour in different Year 5 classes.

- Levels of student engagement were found to be relatively high and classroom climates were generally positive. Teacher detachment and levels of pupil 'off task' behaviour were generally low.

- There was considerable variation in the quality of the educational experiences of children in different classes, indicating that some children attend poorer quality settings, which has implications for the promotion of greater equality of educational opportunities.

- Most teachers broadly followed the format of the National Strategies (Literacy and Maths) except for the use of the plenary which was not observed in nearly half of classes.

- The quality of teaching and pupil response was found to be consistently higher in classes where a plenary was used in both literacy and numeracy lessons and lowest in classes where no plenary was used in either subject.

The impact of School Context

- The incidence of poor pupil behaviour and classroom disorganisation was observed to be greater in schools with higher levels of social disadvantage, measured by the percentage of pupils eligible for free school meals (FSM). The quality of pedagogy was also found to be poorer in schools with higher levels of social disadvantage.

Associations between classroom practice and measures of 'effectiveness'

- Observed practice was found to be better in schools that had been rated more positively by Ofsted Inspectors in earlier inspections (particularly in schools rated more highly on overall leadership and school effectiveness). This suggests that the practice of Year 5 teachers in more effective schools is related to the overall quality of the school and its leadership.

- Significant positive associations were also found between Ofsted judgements of school effectiveness and improvement since the last inspection and teachers' use of a plenary in literacy and numeracy lessons.

Several aspects of observed practice were also found to be weakly related to better value added outcomes in English and Maths. 


\section{The Aims of EPPE 3-11}

This Research Brief focuses on the results of detailed observation of 125 Year 5 classes in a purposive sample of 125 primary schools with a range of effectiveness and from different geographical areas. Here we identify the extent of variations in classroom practice and pupil behaviour and patterns of association with measures of school effectiveness and quality, and with indicators of the social disadvantage of school context.

EPPE 3-11 uses a number of measures of primary school 'effectiveness' and 'quality'. The 'effectiveness' of a school was estimated by comparing linked Key Stage 1 (KS1) to Key Stage 2 (KS2) national assessment results using multilevel 'value added' analyses. The analysis made comparisons between classroom observations, value added measures, and Ofsted judgements of 'effectiveness', 'improvement', 'leadership' and 'quality of teaching and learning' etc., as well as linking contextual information about social disadvantage (\% of pupils' eligible for free school meals [FSM]).

The research tested the applicability, to a large sample of Year 5 classes in England, of two observation instruments designed to measure the quality of practice and range of teacher and pupil behaviours. This allows comparisons with other international research. Observations using the Classroom Observation Schedule (Pianta) instrument were conducted at the start of the morning and afternoon, with a particular focus on literacy and numeracy lessons, although science and other humanities (e.g. history or geography) lessons were also observed. A total of 153, 20 minute literacy and 149 numeracy sessions were observed. A second instrument, the Instructional Effectiveness Instrument (Stipek) was used in 93 classes to observe complete literacy and numeracy lessons (approx. one hour each).

The two instruments identified important differences in observed practice and behaviour and were found to distinguish between better and poorer quality classroom experiences for Year 5 pupils.

\section{Key Findings explored:}

\section{Pedagogical practices and classroom organisation}

Classroom observations showed considerable variation in the quality of learning experiences. The extent of the variation indicates that pupils in different Year 5 classes can have quite different educational experiences. Despite evidence of rising standards across primary schools associated with the National Strategies, it appears that quality remains uneven. This was particularly evident in important domains such as: 'Richness of instructional methods', 'Using basic skills in the context of problem solving' and the 'Development of higher order thinking skills'. Characteristics of classroom that were rated more highly in these areas were: 'thought provoking' reciprocal discussions, children using hypothesis to experiment with a range of ways of tackling a problem and teachers modelling problem solving. In around a fifth of classes relatively little use of evaluative feedback was seen, while approximately $17 \%$ of classes had very low ratings for 'Richness of instruction'. Pupils in such classes therefore had poorer learning environments than those in typical classes.

Teaching analytic skills - There was little or none of this pedagogical practice observed in around $30 \%$ of Year 5 classes in the sample. 'Analysis' in this context includes 'higher order' critical thinking skills of analysis, inference, application, interpretation, problem solving, and planning.

Student engagement - Generally, levels of student engagement were found to be relatively high and classroom climates positive, although in a small minority of classes this was not the case. Teacher detachment was usually low and encouragingly little 'off task' pupil behaviour was 
observed. The levels of 'off task' behaviour observed were lower than those reported in findings from classroom studies conducted in the 1980s.

Organisation of teaching - Overall the time spent in different groupings (e.g. whole class, individual etc.) was found to accord with the National Strategies (Literacy/Numeracy) recommendations for daily lessons, with the exception of the use of the plenary session (see below). More time in whole class (56\%) than individual work $(36 \%)$ was observed, and group work was found to be less common $(9 \%)$. These findings differ from earlier primary school studies where individual work tended to be more common than whole class work, but differences in definitions in different observation instruments make direct comparisons difficult (Galton et al. 1999). Teachers appear to be making more use of 'interactive whole class teaching' as recommended in the National Strategies.

However, some authors, such as Smith et al. (2004), have argued that 'interactive whole class' teaching strategies have not dramatically transformed traditional patterns of whole class interaction. Furthermore, Alexander (2004) suggests that the benefits of 'Dialogic Teaching'11 will only be realised when teachers recognise that it: "...demands both pupil engagement and teacher intervention. And the principle means by which pupils actively engage and constructively intervene is through talk" (Alexander, 2004, authors emphasis).

'Group work' - Observations of pupils in large and small groups were very rare (except in Science where $11 \%$ of observations were in groups). Teachers varied in the extent to which they encouraged pupil co-operation in both the type of tasks they assigned and the level of cooperation they encouraged. The SPRinG study (Blatchford et al, 2004) is an intervention study which promotes positive teacher and pupil interactions. This research indicates that there can be benefits from collaborative group work at KS2 as pupils engage in more (and more sustained) interactions with other pupils. Our research indicates that in many of the Year 5 classes observed such group work was uncommon.

\section{The Plenary}

Most schools broadly followed the format of the National Strategies (Literacy and Maths) except for the use of plenary. Around a third of classes observed used a plenary in both literacy and numeracy. In half the classes a plenary was observed in one but not the other subject. In approximately a quarter no plenary was observed in either lesson. The quality of teaching was found to be significantly higher in classes where plenaries were used for both literacy and numeracy, and lowest in classes where plenaries were absent. The absence of the plenary in around half of literacy and numeracy lessons observed (51\% Literacy, $49 \%$ numeracy) is of particular concern as this part of the lesson is intended to give opportunities for feedback for improvement and consolidation of learning. Black and Wiliam (1998) argue that 'informative' feedback is 'an essential component of classroom work' (1998 p9) that can lead to raised standards of achievement. By missing this part of the lesson some teachers may be reducing the opportunity to provide consolidation. In particular the use of more demanding higher order communication is typically more common in plenary and other whole class activities.

\section{Disadvantaged groups}

The quality of teaching tends to be poorer in schools with higher levels of social disadvantage and this has implications for the social inclusion and raising standards agenda. In such schools, Year 5 classes scored significantly lower on particular aspects of the 'quality' of teaching. In mathematics, there were fewer opportunities for pupils to practice basic skills in the context of problem solving (which encourages higher order thinking). There was also less 'social support

\footnotetext{
${ }^{11}$ Broadly equivalent conceptions include 'mutualist and dialectical pedagogy' (Bruner), 'dialogic enquiry' (Wells), 'interthinking' (Mercer), 'dialogue of enquiry' (Lindfor), and in the early years, 'sustained shared thinking' (Siraj-Blatchford, et al, (2002).
} 
for learning' characterised by everyone's contribution being taken seriously and pupil errors being used as opportunities to explore learning. There were also fewer opportunities for children to demonstrate their subject knowledge.

In literacy, pupils in schools serving more disadvantaged intakes spent more time in 'off task' talk (student engagement) and their classrooms were less likely to be well organised, with transitions between activities being poorly managed. In addition, the classroom climate (extent to which pupils are respected and have autonomy) and social support for learning (high expectations) were significantly and negatively associated with the level of social disadvantage (measured by \% of pupils eligible for FSM).

The classroom observations that identified disruptive behaviour, discipline episodes and class 'chaos' suggest that pupil behaviour was generally good in the large majority of classes. However, behaviour tended to be worse in schools where there were relatively more children eligible for free school meals (\% FSM). The results also indicated that poor organisation of work and classes by some Year 5 teachers was associated with level of social disadvantage (e.g. on the item 'chaos').

These results warrant further investigation, given concerns about the gap in attainment related to pupil background which has been shown to increase as children progress through school. Our findings may reflect the influence of lower teacher expectations or the recruitment of less experienced or poorly performing teachers in schools serving more disadvantaged communities. They may also link to difficulties relating to pupil behaviour, attitudes and attendance. In fact it may be all of these, 'expectations' do not have to be 'self-fulfilling' to constitute a problem, as Good and Brophy (1997) have argued: "Expectations tend to be selfsustaining. They affect both perception, by causing teachers to be alert for what they expect and less likely to notice what they do not expect, and interpretation, by causing teachers to interpret (and perhaps distort) what they see so that it is consistent with their expectations. Some expectations persist even though they do not coincide with the facts (Good \& Brophy, 1990, p441).

Associations between classroom practice and measure of 'effectiveness'

One method used in the EPPE research to explore the 'effectiveness' of all primary schools (over 16,000) in England was to analyse matched pupil national assessment scores from KS1 to KS2 taking account of the background characteristics of the child and the school (a form of contextualised value added based on data for 2002-4). The analysis is therefore based on the 'residual' scores for each school in the three core subjects (English, maths and science calculated separately). The value added results were then extracted and compared with the observation sample of 125 focal schools. This identified schools where children generally made progress 'as expected', 'better than' or 'less well' than predicted by their prior attainment and background.

Moderately strong 'between-subject' statistical results were identified indicating schools that were more effective in one core subject tend to be more effective in others, while those that are less effective in one area also tend to be less effective in others. In addition the extent of stability in school effectiveness was investigated across years. Several aspects of teachers' observed practices were found to be significantly associated with the value added analyses of progress in Maths and English in KS2.

Although only weak to moderate, such associations show some interesting links between overall school effectiveness and specific features of classroom practices. Thus they provide some helpful insights into what feature of effective practice and pedagogical approaches may promote better pupil progress. 
In English three aspects of pedagogy were noteworthy: having a positive classroom climate (safe and respectful, opportunities for collaboration, sensitive discipline); good classroom routines (maximised use of instructional time, well prepared materials) and the developing of higher order thinking skills in the context of 'reading as meaning making'. This involved teachers encouraging children when reading to go beyond decoding text, and teachers drawing on pupils' previous knowledge and reasoning to encourage pupils to evaluate critically their comprehension.

In Maths, as well as having good classroom routines, three other areas of mathematical pedagogical knowledge were associated with better outcomes in the value added measures: 'use of maths analyses', 'depth of knowledge' and 'locus of maths authority'. In 'use of maths analyses' higher ratings were associated with children being given opportunities to construct original ways to solve maths problems and being allowed to make conjectures with justifications. 'Depth of knowledge' focused on the depth to which maths knowledge is treated in class. This was evident when teachers structured their lessons so that most children were engaged in one or more of the following: demonstrating their understanding of the problematic nature of information or ideas, demonstrating complex understandings by arriving at a reasoned, supported conclusion or explaining how they solved a complex problem. The 'locus of maths authority' is the extent to which the teacher and the pupil hold each other accountable for convincing themselves and each other that their reasoning is sound. In classrooms where this occurred the teacher often answered a question with a question or offered instrumental help (good scaffolding), pushing pupils to make their own decisions.

The second method of exploring 'effectiveness' and its relationship to classroom practices was to compare the observations of our 125 'focal' schools with Ofsted judgements of quality of provision from the most recent inspection reports (conducted prior to the observation data collection). Classroom observations were matched with Ofsted global judgements on overall school 'effectiveness', 'improvement since last inspection', 'leadership', 'quality of teaching and learning' and inspectors' ratings of pupil outcomes (attendance, attitudes and behaviour). The results indicated significant, though modest positive associations with a number of our observational measures of teacher pedagogy and teacher and pupil behaviour. We found that teachers' observed practice in Y5 classes tended to be 'better' in those schools that had previously been rated more positively in the professional judgement of inspectors. Of particular note were the positive associations between Ofsted judgements and higher observed ratings of 'productive use of instructional time' (smooth transitions, good planning, efficient routines for when pupils finish work), 'pupil self reliance' (where pupils display autonomy, initiative and are self directed), 'richness of instructional methods' (intellectually engaging and thought provoking lessons which contain reciprocal discussions) and 'positive classroom climate' (where the emotional and social tone of the classroom was respectful, safe and welcoming).

The positive associations between the quality of overall school leadership and better classroom practice identified by observations supports the view that overall school influences can affect classroom practice and the conclusion that good school leadership tends to promote better teaching and learning and thus better outcomes for children. Generally the findings indicate links between more global constructs of school effectiveness, as defined by inspectors and more specific aspects of observed classroom practice related to the quality of teaching. The classroom practices of individual teachers appear to be positively influenced directly or indirectly by the overall effectiveness and leadership of their school.

The correlations between Ofsted ratings of quality and the 'value added' indicators with the classroom level observations indicate that the classroom observation schedules used by researchers in the 125 'focal' schools allow important features of classroom practice associated with better outcomes for children in English primary schools to be identified. These analyses 
improve understanding of the extent of variation in school and classroom processes in Year 5, and provides insights into more effective practices and pedagogical approaches.

\section{Conclusions and Key Messages}

This research brief highlights new findings on the quality of teaching and learning in Year 5 English primary classes. The results are relevant to policy makers and practitioners concerned with improving practice and promoting greater equity by closing the attainment gap associated with social disadvantage. There are implications for the further development of the National Strategies and the results highlight areas of possible weakness in the teaching in some classes that could benefit from further guidance and professional development. The findings are of relevance to the Excellence and Enjoyment (DfES, 2003) agenda and the promotion of personalised learning. They should also to be of interest to Ofsted inspectors and to schools' approaches to the improvement of classroom practice through self evaluation and review.

\section{Key messages}

- There is wide variation in teachers' practice and children's responses in Year 5 classes and this is likely to affect pupils' educational outcomes.

- The quality of classroom practice is associated with the use of plenary sessions in literacy and numeracy lessons. Practice was found to be better in classes that used plenaries in both of these subjects and poorer in classes where no plenary was observed in either. Plenaries were present in only approximately half of the literacy and numeracy lessons observed.

- The quality of Year 5 pedagogy and organisation and pupil behaviour is poorer in schools with higher levels of social disadvantage in their pupil intakes. This may reflect lower expectations, difficulties in recruiting/retaining good/experienced teachers and the greater behavioural difficulties associated with teaching in more challenging contexts. The quality of Year 5 practice observed was better in schools that had been rated more highly in terms of overall school leadership, effectiveness and improvement on the previous inspection. Such schools appear to provide a more positive context for teaching and learning.

- The use of well researched classroom observation instruments may provide valuable evidence for teachers' professional development and support a school's self evaluation and review process. This is particularly relevant where they identify quality across a range of features of teaching and learning that are linked with better outcomes for children

\section{Methodology}

The EPPE 3-11 project contains a series of three 'nested' studies or 'tiers' which help answer specific research questions (www.ioe.ac.uk/projects/eppe).

Tier 1 helps answer the research question about the effectiveness of the $800+$ primary schools the EPPE3-11 children attend. It uses statistical data (KS1 and KS2 national assessment results) derived from every primary school in the country (over three consecutive years 20022004) for English and Maths so that schools can be studied in various 'effectiveness' bands (the 'value-added analyses). Further information on Tier 1 can be found in Melhuish et al (2006). Tier 3, the focus of this Research Brief, explores classroom practice through two different but complementary classroom observations. This addresses the question of what constitutes good classroom practice in Year 5 and what makes for an effective primary school experience.

Informed by the Tier 1 analyses, this stage of the project links observations of classroom practice to a range of ratings of effectiveness and quality. A sample of 125 'focal' schools' were selected from the Tier 1 analyses. The schools were chosen across the range of 'effectiveness' 
scores for English and Maths and in different LEAs across the country from amongst those attended by children in the EPPE3-11 sample. Trained researchers conducted detailed classroom observations in each school in 2004 and 2005. Classroom observations were conducted using schedules developed in the USA for the NICHD study. These two schedules (Pianta NICHD 2001 and Stipek 1999, named after their authors) covered a wide range of pedagogical practices and pupil behaviours. The frequency of different behaviours was observed for individual pupils as well as more global rating of general classroom quality. For further description of the research instruments see Sammons et al. (2006a \& b). In addition to the value added analyses described above, the classroom observations were also compared to Ofsted inspection judgements on effectiveness, improvement since last inspection, leadership, quality of teaching and learning and inspectors' ratings of a number of pupil outcomes (attendance, attitudes and behaviour) using numeric judgements matched to 107 of the focal schools.

\section{References:}

Alexander, R. (2004) Towards dialogic teaching: rethinking classroom talk, York, Dialogos

Black, P. \& Wiliam, D. (1998), 'Assessment and classroom learning'. Assessment in Education: Principals, Policy and Practice, 5, 7-75.

Galton, M., Hargreaves, L., Comber, C., Wall, D. \& Pell, A. (1999), Inside the Primary Classroom: 20 Years On. London: Routledge.

Blatchford, P., Galton, M. \& Kutnick, P. (2004) Improving the effectiveness of pupil group work. Report on first results from the TLRP Phase 2 SPRinG project, Paper to Annual ESRC TLRP Conference, Cardiff, November 2004.

Good, T.L. \& Brophy, J.E. (1990) Educational Psychology: A realistic approach $4^{\text {th }}$ ed. London: Longman.

NICHD. (2001), 'Fifth Grade School Observation Procedures Manual.' NICHD Study of Early Child Care and Youth Development: NICHD Study of Early Child Care and Youth Development.

Melhuish, E., Romaniuk, H., Sammons, P., Sylva, K., Siraj-Blatchford I \& Taggart B (2006) Effective Pre-school and Primary Education 3-11(EPPE 3-11) project. The Effectiveness of Primary Schools in England in Key Stage 2 for 2002, 2003 and 2004. DfES Research Brief X06-06. Nottingham: DfES.

Sammons, P., Taggart, B., Siraj-Blatchford, I., Sylva, S., Melhuish, E. Barreau, S. \& Manni. L. (2006a) Effective Pre-school and Primary Education (EPPE 3-11) Project: Variations in Teacher and Pupil Behaviours in Year 5 Classrooms and Associations with School Characteristics. DfES Research Report RR817. Nottingham: DfES.

Sammons, P., Taggart, B., Sylva, K., Melhuish, E., Siraj-Blatchford, I., Barreau, S. and Manni, L. (2006b), Effective Pre-school and Primary Education 3-11 Project (EPPE 3-11) Tier 3: Variations in Teacher and Pupil Behaviours in Year 5 Classes. Full Report. Institute Of Education, University of London. http://www.ioe.ac.uk/schools/ecpe/eppe/eppe3-11/eppe3-11pubs.htm

Siraj-Blatchford, I., Sylva, K., Muttock, S., Gilden, R., \& Bell, D. (2002). Researching effective pedagogy in the early years. DfES Research Report 356. Nottingham: Department for Education and Skills.

Smith, F., Hardman, F., Wall, K. and Mroz, M. (2004), 'Interactive Whole Class Teaching in the National Literacy and Numeracy Strategies'. British Educational Research Journal, 30, 395-411.

Stipek, D. (1999), Instructional Environment Observation Scale. University of California: MacArthur Pathways through Middle Childhood Network. 


\section{Appendix 2 The classroom observation instruments:}
a) The Classroom Observation System for Fifth Grade (COS-5)
b) The Instructional Environment Observation (IEO), Literacy
c) The Instructional Environment Observation (IEO), Numeracy 
COS-5 - Frequency of Behaviour

\begin{tabular}{|c|c|c|c|c|c|c|c|c|c|c|}
\hline \multirow{2}{*}{$\begin{array}{l}1 \text { Child-level setting } \\
\text { Whole class }\end{array}$} & \multicolumn{10}{|c|}{ Minute interval } \\
\hline & 1 & 2 & 3 & 4 & 5 & 6 & 7 & 8 & 9 & 10 \\
\hline & 1 & 2 & 3 & 4 & 5 & 6 & 7 & 8 & 9 & 10 \\
\hline Small group 6 or fewer & 1 & 2 & 3 & 4 & 5 & 6 & 7 & 8 & 9 & 10 \\
\hline Individual & 1 & 2 & 3 & 4 & 5 & 6 & 7 & 8 & 9 & 10 \\
\hline \multicolumn{11}{|l|}{11 Content of tc activity } \\
\hline Literacy/Language Arts & 1 & 2 & 3 & 4 & 5 & 6 & 7 & 8 & 9 & 10 \\
\hline Word-level Activities & 1 & 2 & 3 & 4 & 5 & 6 & 7 & 8 & 9 & 10 \\
\hline Comprehension & 1 & 2 & 3 & 4 & 5 & 6 & 7 & 8 & 9 & 10 \\
\hline $\begin{array}{l}\text { Part of Literacy hour } 12234^{*} \\
\end{array}$ & $\overline{11}$ & 2 & $\overline{3}$ & 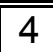 & $\overline{5}$ & 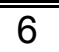 & $\overline{7}$ & $\overline{8}$ & $\overline{9}$ & 10 \\
\hline Part of Literacy hour $\mathrm{W} / \mathrm{S} / \mathrm{T}$ & 1 & 2 & 3 & 4 & 5 & 6 & 7 & 8 & 9 & 10 \\
\hline Mathematics & 1 & 2 & 3 & 4 & 5 & 6 & 7 & 8 & 9 & 10 \\
\hline Computation & 1 & 2 & 3 & $\overline{4}$ & 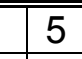 & 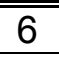 & 7 & 8 & 9 & 10 \\
\hline Concept Development/Problem Solving & 1 & 2 & 3 & 4 & 5 & 6 & 7 & 8 & 9 & 10 \\
\hline Part of NNS O / M / $\mathrm{P}^{*}$ & 1 & 2 & 3 & 4 & 5 & 6 & 7 & 8 & 9 & 10 \\
\hline Science & $\overline{11}$ & $\overline{2}$ & 3 & 4 & 5 & 6 & 7 & 8 & 9 & 10 \\
\hline Humanities & 1 & 2 & 3 & 4 & 5 & 6 & 7 & 8 & 9 & 10 \\
\hline Enrichment & 1 & 2 & 3 & 4 & 5 & 6 & 7 & 8 & 9 & 10 \\
\hline Computers/Technology & 1 & 2 & 3 & 4 & 5 & 6 & 7 & 8 & 9 & 10 \\
\hline Free time & 1 & 2 & 3 & 4 & 5 & 6 & 7 & 8 & 9 & 10 \\
\hline Transitions/Management/Business & 1 & 2 & 3 & 4 & 5 & 6 & 7 & 8 & 9 & 10 \\
\hline \multicolumn{11}{|l|}{111 Teacher Behaviour } \\
\hline Attends to TC & 1 & 2 & 3 & 4 & 5 & 6 & 7 & 8 & 9 & 10 \\
\hline Teaching Basic Skills/facts & 1 & 2 & 3 & 4 & 5 & 6 & 7 & 8 & 9 & 10 \\
\hline Teaching Analysis/Infer/Plan & 1 & 2 & 3 & 4 & 5 & 6 & 7 & 8 & 9 & 10 \\
\hline Managerial instructions & 1 & 2 & 3 & 4 & 5 & $\overline{6}$ & 7 & 8 & 9 & 10 \\
\hline Monitoring/Checking work & 1 & 2 & 3 & 4 & 5 & 6 & 7 & 8 & 9 & 10 \\
\hline Display Positive Affect & 1 & 2 & 3 & 4 & 5 & 6 & 7 & 8 & 9 & 10 \\
\hline Displays Negative Affect & 1 & 2 & 3 & 4 & 5 & 6 & 7 & 8 & 9 & 10 \\
\hline Disciplines & 1 & 2 & 3 & 4 & 5 & 6 & 7 & 8 & 9 & 10 \\
\hline \multicolumn{11}{|l|}{ 1V Child Academic Behaviour } \\
\hline Engaged in learning & 1 & 2 & 3 & 4 & 5 & 6 & 7 & 8 & 9 & 10 \\
\hline Highly Engaged & 1 & 2 & 3 & 4 & 5 & 6 & 7 & 8 & 9 & 10 \\
\hline Unproductive/Spaced Out /Disengaged & 1 & 2 & 3 & 4 & 5 & 6 & 7 & 8 & 9 & 10 \\
\hline Off-task - Alternative Academic Activity & 1 & 2 & 3 & 4 & 5 & 6 & 7 & 8 & 9 & 10 \\
\hline Learning/Performing Basic Skills/Facts & 1 & 2 & 3 & 4 & 5 & 6 & 7 & 8 & 9 & 10 \\
\hline Learning/Performing Analysis/Inference etc. & 1 & 2 & 3 & 4 & 5 & 6 & 7 & 8 & 9 & 10 \\
\hline Collaborative Work & $\overline{11}$ & $\overline{2}$ & 3 & 4 & $\overline{5}$ & 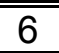 & $\overline{77}$ & 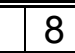 & 9 & 10 \\
\hline Requests Attention/Help/Information & 1 & 2 & 3 & 4 & 5 & 6 & 7 & 8 & 9 & 10 \\
\hline Volunteers & 1 & 2 & 3 & 4 & 5 & 6 & 7 & 8 & 9 & 10 \\
\hline \multicolumn{11}{|l|}{ V Child Social Behaviour } \\
\hline Positive /Neutral Engagement with Peers & 1 & 2 & 3 & 4 & 5 & 6 & 7 & 8 & 9 & 10 \\
\hline $\begin{array}{l}\text { Negative/Aggressive Engagement with } \\
\text { Peers }\end{array}$ & 1 & 2 & 3 & 4 & 5 & 6 & 7 & 8 & 9 & 10 \\
\hline Positive Affect Toward Teacher & 1 & 2 & 3 & 4 & 5 & 6 & 7 & 8 & 9 & 10 \\
\hline Negative Affect Toward Teacher & 1 & 2 & 3 & 4 & 5 & 6 & 7 & 8 & 9 & 10 \\
\hline General Disruptive Behaviour & 1 & 2 & 3 & 4 & 5 & 6 & 7 & 8 & 9 & 10 \\
\hline $\begin{array}{l}\text { End-of-behavioural ratings: } \\
\text { Classroom level setting } \\
\text { Teacher suggests/offers collaborative activity } \\
\text { Test/Quiz administered during any portion of } \\
\text { (Adapted from NICHD FSV01G5) }\end{array}$ & iole, & & A lo & & $\begin{array}{l}3 \ldots . . . \\
N \\
N\end{array}$ & ewh & & & & \\
\hline
\end{tabular}


A Measures of Quality

Child Codes

\begin{tabular}{|l|l|l|l|l|l|l|}
\hline 1 & 2 & 3 & 4 & 5 & 6 & 7 \\
\hline Uncharacteristic & & $\begin{array}{l}\text { Minimally } \\
\text { characteristic }\end{array}$ & $\begin{array}{l}\text { Norm } \\
\text { Exception: 5 }\end{array}$ & $\begin{array}{l}\text { Very } \\
\text { characteristic }\end{array}$ & & $\begin{array}{l}\text { Extremely } \\
\text { characteristic }\end{array}$ \\
\hline
\end{tabular}

Child code Scores should reflect global classroom observations related to TC's point of $\quad$ Rating view.

1 Positive Affect (1-7)

2 Self Reliance (1-7)

\begin{tabular}{l|l|l|l|l|l|l|}
1 & 2 & 3 & 4 & 5 & 6 & 7
\end{tabular}

3 Sociable/Cooperative with Peers (1-7)

\begin{tabular}{llllllll|}
1 & 2 & 3 & 4 & 5 & 6 & 7
\end{tabular}

4 Attention (1-7)

\begin{tabular}{l|l|l|l|l|l|l|}
1 & 2 & 3 & 4 & 5 & 6 & 7
\end{tabular}

5 Disruptive (1-7)

6 Activity Level (1-7)

\begin{tabular}{|l|l|l|l|l|l|l|}
\hline 1 & 2 & 3 & 4 & 5 & 6 & 7 \\
\hline
\end{tabular}

7. Child-Teacher Relationship (1-7)

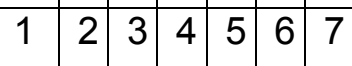

\section{End-of-cycle ratings:}

\begin{tabular}{|c|c|c|c|c|}
\hline \multicolumn{5}{|c|}{ General Observations on the whole class } \\
\hline 1 & The children respected by peers. & $\begin{array}{l}1 \text { None/Some } \\
\text { of the time }\end{array}$ & 2 Most of the time & $\begin{array}{l}3 \text { All of the } \\
\text { time }\end{array}$ \\
\hline 2 & $\begin{array}{l}\text { Children are responsible for time and materials } \\
\text { (independence) tangible responsibility }\end{array}$ & $\begin{array}{l}1 \text { None/Some } \\
\text { of the time }\end{array}$ & 2 Most of the time & $\begin{array}{l}3 \text { All of the } \\
\text { time }\end{array}$ \\
\hline 3 & $\begin{array}{l}\text { The learning intentions of the lesson/activity is } \\
\text { clear to children }\end{array}$ & 1 Not clear & 2 Clear to some & 3 Clear to all \\
\hline 4 & $\begin{array}{l}\text { Children could reflect on their learning through } \\
\text { review }\end{array}$ & 1 No evidence & 2 Some evidence & 3 Very evident \\
\hline 5 & $\begin{array}{l}\text { The teacher's materials/resources were well } \\
\text { organised/managed and 'fit for purpose' }\end{array}$ & $\begin{array}{l}1 \text { Not well } \\
\text { organised }\end{array}$ & $\begin{array}{l}2 \text { Some } \\
\text { organisation }\end{array}$ & $\begin{array}{l}3 \text { Well } \\
\text { organised }\end{array}$ \\
\hline
\end{tabular}

(Adapted from NICHD FSV01G5) 
B Measures of Quality

Classroom Codes

\begin{tabular}{|l|l|l|l|l|l|l|}
\hline 1 & 2 & 3 & 4 & 5 & 6 & 7 \\
\hline Uncharacteristic & & $\begin{array}{l}\text { Minimally } \\
\text { characteristic }\end{array}$ & Norm & $\begin{array}{l}\text { Very } \\
\text { characteristic }\end{array}$ & & $\begin{array}{l}\text { Extremely } \\
\text { characteristic }\end{array}$ \\
\hline
\end{tabular}

Classroom codes Scores should reflect global classroom observations

1 Richness of instructional methods (1-7)

2 Over-Control (1-7)

3 Chaos (1-7)

4 Detachment / Teacher (1-7)

5 Positive Classroom Climate (1-7)

6 Negative Classroom Climate (1-7)

7 Productive Use of Instructional Time (1-7)

8 Evaluative Feedback (1-7)

9 Teacher Sensitivity (1-7)

\begin{tabular}{l|l|l|l|l|l|}
\hline 1 & 2 & 3 & 4 & 5 & 6
\end{tabular}

Rating

End-of-cycle ratings:

1 The teacher is clear about what she expects the children to do in their activities.

\begin{tabular}{|l|l|l|l|l|}
\hline 1 & 2 & 3 & 4 & 5 \\
\hline $\begin{array}{l}\text { Not clear, very } \\
\text { confusing to all }\end{array}$ & $\begin{array}{l}\text { Clearer but, majority } \\
\text { of children still } \\
\text { confused }\end{array}$ & $\begin{array}{l}\text { Some clear and some } \\
\text { confused }\end{array}$ & Mostly clear & Extremely clear to all \\
\hline
\end{tabular}

2 The teacher ensures that concepts/ideas are clear to the children.

\begin{tabular}{|l|l|l|l|l|}
\hline 1 & 2 & 3 & 4 & 5 \\
\hline $\begin{array}{l}\text { Not clear, very } \\
\text { confusing to all }\end{array}$ & $\begin{array}{l}\text { Clearer but, majority } \\
\text { of children still } \\
\text { confused }\end{array}$ & $\begin{array}{l}\text { Some clear and some } \\
\text { confused }\end{array}$ & Mostly clear & $\begin{array}{l}\text { Extremely clear to all } \\
\text { (Excluding SEN } \\
\text { child) }\end{array}$ \\
\hline
\end{tabular}


The Effectiveness of Pre-School and Primary Education 3-11 (EPPE 3-11)

Observational Ratings of Year 5 Classrooms (Summer 2005)

Literacy Stipek

Name of Researcher. Code No

Name of School. Code No

Name of Teacher. Code No.

Observation Number (should be coder no. / chronological obs no.).

Observing: Literacy (specify set if applicable)

Date: .

1. Classroom personnel

Total number of adults during observation $\begin{array}{lllllllllll}0 & 1 & 2 & 3 & 4 & 5 & 6 & 7 & 8 & 9 & 10\end{array}$

\begin{tabular}{|l|l|l|}
\hline \multicolumn{2}{|l|}{ Description of each Adult } & Definition no. from list below \\
\hline 1 & & \\
\hline 2 & & \\
\hline 4 & & \\
\hline 5 & & \\
\hline 6 & & \\
\hline
\end{tabular}

Adult definition number: Enter each person's number in the Definitions column above.

\begin{tabular}{|l|l|l|}
\hline Teaching staff & Support staff & Others \\
\hline 1 Class teacher & 4 Teachers' Aide/ primary helper & 7 Student teacher \\
\hline 2 Regular teacher & 5 Learning support assistant (general) & 8 Volunteer \\
\hline 3 Special needs teacher & 6 Learn. Supp. Assistant (individ child) & \\
\hline Any other please specify: & \multicolumn{2}{|l}{} \\
\hline
\end{tabular}

2. Number of children Number of boys

3. Type of classroom - please ring

\begin{tabular}{|l|l|l|}
\hline 1 Regular classroom & 4 Library & 7 Hall \\
\hline 2 Resource room & 5 Music Room & 8 Playground \\
\hline 3 Computer room & 6 Art Room & \\
\hline Any other please specify: &
\end{tabular}

4. Time observation begin

$\begin{array}{lllllllllllllllll}\text { Hours: } & 8 & 9 & / & 10 & 11 & / 2 & / & 1 & 2 & / & 3 & / & 4 \\ \text { Minutes } & 0 & / & / & 2 & / & 3 & / & 4 & / & 5 & & & & & & \\ & 0 & 1 & / & 2 & / & 3 & / & 4 & 5 & / & 6 & 7 & / & 8 & \end{array}$

5. Time Observation Ends:
Hours :
$8 / 9 / 10 / 11 / 12 / 1 / 2 / 3 / 4$
Minutes
$0 / 1 / 2 / 3 / 4 / 5$
$0 / 1 / 2 / 3 / 445 / 6 / 7 / 8 / 9$

Subject: Literacy

Observer Number

Collect a copy of time table

Information on classroom setting and pupil organisation

Describe grouping: structure of class/pupils:

Single academic year group, ordinary mixed ability or 
Mixed Year $5 / 6$ children, but set for this subject and it is the middle group of Year $5 / 6$ s combined Single academic year group, but streamed and the mixed ability group

Purpose of lesson: learning intention as articulated by the teacher

Describe the type of text children are reading:

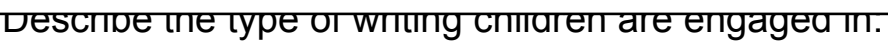

Did you see the following? Please check all that apply.

Whole class reading

d) Individual work

Whole class writing

e) Teacher sanctioned group work (collaborative)

Main lesson f) Plenary $\square$ 
Observer Number

Subject: Literacy

SCORING SHEETS

\begin{tabular}{||l|l||}
\hline \multicolumn{1}{|c|}{} & $\begin{array}{l}\text { Scor } \\
\text { e }\end{array}$ \\
\hline $\begin{array}{l}\text { General Classroom Management and Climate Scales } \\
\text { One for each of the lessons observed }\end{array}$ & \\
\hline \hline A Classroom climate & \\
\hline B Classroom routines & \\
\hline
\end{tabular}

General Instruction Scales

One for each of the lessons observed

C Cross-Disciplinary Connections

D Linkage to life beyond the classroom

E Social support for student learning

F $\quad$ Student engagement 
Observer Number

Subject: Literacy

\section{Reading Instructional Scales}

$\mathrm{L} \quad$ Reading as meaning making

M Basic skills development in the context of reading

Writing Instructional Scales

$\mathrm{N} \quad$ Higher order thinking in writing

O Purposeful development of writing skills

Instructional Conversations

$\mathrm{P} \quad$ Instructional conversations 
The Effectiveness of Pre-School and Primary Education 3-11 (EPPE 3-11)

Observational Ratings of Year 5 Classrooms (Summer 2005)

NUMERACY Stipek

Name of Researcher. Code No

Name of School. Code No

Name of Teacher. Code No.

Observation Number (should be coder no. / chronological obs no.).

Observing: Numeracy (specify set if applicable)

Date: $. . . . / \ldots \ldots / \ldots \ldots$

1. Classroom personnel

Total number of adults during observation $\begin{array}{lllllllllll}0 & 1 & 2 & 3 & 4 & 5 & 6 & 7 & 8 & 9 & 10\end{array}$

\begin{tabular}{|l|l|l|}
\hline \multicolumn{2}{|l|}{ Description of each Adult } & Definition no. from list below \\
\hline 1 & & \\
\hline 2 & & \\
\hline 3 & & \\
\hline 5 & & \\
\hline 6 & & \\
\hline
\end{tabular}

Adult definition number: Enter each person's number in the Definitions column above.

\begin{tabular}{|l|l|l|}
\hline Teaching staff & Support staff & Others \\
\hline 1 Class teacher & $\begin{array}{l}4 \text { Teachers' Aide/ primary } \\
\text { helper }\end{array}$ & 5 Student teacher \\
\hline 2 Regular teacher & $\begin{array}{l}\text { 5 Learning support assistant } \\
\text { (general) }\end{array}$ & 8 Volunteer \\
\hline 3 Special needs teacher & $\begin{array}{l}6 \text { Learn. Supp. Assistant } \\
\text { (individ child) }\end{array}$ & \\
\hline Any other please specify: & & \\
\hline
\end{tabular}

2. Number of children Number of boys.

3. Type of classroom - please ring

\begin{tabular}{|l|l|l|}
\hline 1 Regular classroom & 4 Library & 7 Hall \\
\hline 2 Resource room & 5 Music Room & 8 Playground \\
\hline 3 Computer room & 6 Art Room & \\
\hline Any other please specify: &
\end{tabular}

4. Time observation begin
Hours :
$8 / 9 / 10 / 11 / 12 / 1 / 2 / 3 / 4$
Minutes
$0 / 1 / 2 / 3 / 4 / 5$
$0 / 1 / 2 / 3 / 4 / 5 / 6 / 7 / 8 / 9$

5. Time Observation Ends:
Hours :
$8 / 9 / 10 / 11 / 12 / 1 / 2 / 2 / 3 / 4$
Minutes
$0 / 1 / 2 / 3 / 4 / 5$

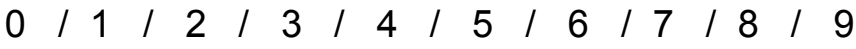


Collect a copy of time table

Information on classroom setting and pupil organisation

Describe grouping: structure of class/pupils:

Single academic year group, ordinary mixed ability or

Mixed Year 5/6 children, but set for this subject and it is the middle group of Year $5 / 6$ s combined

Single academic year group, but streamed and the mixed ability group

Purpose of lesson: learning intention as articulated by the teacher

Did you see the following? Please check all that apply.

Oral/Mental session

Main lesson $\square$

Individual work $\square$

Teacher sanctioned group work (collaborative)

Plenary $\square$ 
Observer Number

Subject: Numeracy

SCORING SHEETS

\begin{tabular}{|l|l|}
\hline & $\begin{array}{l}\text { Scor } \\
\text { e }\end{array}$ \\
\hline General Classroom Management and Climate Scales
\end{tabular}

One for each of the lessons observed

A Classroom climate

B Classroom routines

General Instruction Scales

One for each of the lessons observed

C Cross-Disciplinary Connections

D Linkage to life beyond the classroom

E Social support for student learning

F Student engagement 
Observer Number

Subject: Numeracy

\begin{tabular}{|c|c|}
\hline & matical Instructional Scales \\
\hline $\bar{G}$ & Use of maths analysis \\
\hline $\mathrm{H}$ & Depth of knowledge and student understanding \\
\hline 1 & Basic skill development in the context of problem solving \\
\hline $\mathrm{J}$ & Maths discourse and communication \\
\hline $\mathrm{K}$ & Locus of maths authority \\
\hline
\end{tabular}


Appendix 3 Complete List of Schools

(Including Quality of Pedagogy, Academic Effectiveness Scores* and FSM)

\begin{tabular}{|c|c|c|c|c|c|}
\hline School & IEO & $\cos -5$ & $\begin{array}{l}\text { Maths Residual } \\
\text { Score }\end{array}$ & $\begin{array}{l}\text { English } \\
\text { Residual } \\
\text { Score }\end{array}$ & $\begin{array}{l}\% \text { Pupils Eligible } \\
\text { for FSM }\end{array}$ \\
\hline \multicolumn{6}{|c|}{ Academically Highly Effective, Good Quality pedagogy } \\
\hline s01 & 3 & 5 & 3 & 4 & 0.00 \\
\hline S02 & 3 & 3 & 5 & 4 & 21.62 \\
\hline S03 & 2 & 5 & 4 & 4 & 31.51 \\
\hline S04 & 3 & 4 & 3 & 4 & 30.80 \\
\hline S05 & 3 & 4 & 3 & 5 & 15.94 \\
\hline S06 & 3 & 3 & 5 & 3 & 2.94 \\
\hline S07 & 3 & 4 & 3 & 4 & 18.30 \\
\hline S08 & 3 & 4 & 4 & 4 & 11.84 \\
\hline S12 & 3 & 3 & 3 & 4 & 30.66 \\
\hline S13 & 3 & 4 & 4 & 3 & 49.13 \\
\hline \multicolumn{6}{|c|}{ Medium Academic Effectiveness, Medium Quality pedagogy } \\
\hline S25 & 2 & 3 & 3 & 3 & 7.19 \\
\hline S26 & 2 & 3 & 3 & 3 & 11.41 \\
\hline S27 & 2 & 3 & 3 & 3 & 5.19 \\
\hline S28 & 2 & 3 & 3 & 3 & 2.53 \\
\hline S29 & 2 & 3 & 3 & 3 & 18.50 \\
\hline S30 & 2 & 3 & 3 & 3 & 35.19 \\
\hline S31 & 2 & 3 & 3 & 3 & 15.00 \\
\hline S32 & 2 & 3 & 3 & 3 & 9.01 \\
\hline S36 & 2 & 3 & 3 & 3 & 13.74 \\
\hline \multicolumn{6}{|c|}{ Low Academic Effectiveness, Poor Quality pedagogy } \\
\hline S73 & 1 & 3 & 2 & 3 & 7.63 \\
\hline S74 & 2 & 3 & 2 & 2 & 10.39 \\
\hline S75 & 2 & 3 & 2 & 2 & 39.13 \\
\hline S76 & 1 & 1 & 1 & 2 & 53.11 \\
\hline S77 & 2 & 1 & 2 & 2 & 12.31 \\
\hline S78 & 2 & 1 & 3 & 2 & 12.63 \\
\hline S79 & 2 & 3 & 2 & 2 & 59.61 \\
\hline S80 & 1 & 2 & 2 & 1 & 40.38 \\
\hline S81 & 1 & 1 & 2 & 3 & 15.04 \\
\hline S82 & 1 & 1 & 3 & 2 & 15.68 \\
\hline
\end{tabular}

Continued next page 


\begin{tabular}{|c|c|c|c|c|c|c|}
\hline School & IEO & COS -5 & $\begin{array}{l}\text { Maths } \\
\text { Score }\end{array}$ & Residual & $\begin{array}{l}\text { English } \\
\text { Residual } \\
\text { Score }\end{array}$ & FSM \\
\hline \multicolumn{7}{|c|}{ High/Medium Academic Effectiveness, Good/Medium Quality pedagogy } \\
\hline So9 & 2 & 4 & 4 & & 3 & 21.13 \\
\hline S10 & 2 & 4 & 4 & & 3 & 7.01 \\
\hline S11 & 2 & 3 & 3 & & 4 & 41.52 \\
\hline S14 & 3 & 4 & 3 & & 3 & 11.04 \\
\hline S15 & 3 & 4 & 3 & & 3 & 9.01 \\
\hline S16 & 3 & 4 & 3 & & 3 & 4.15 \\
\hline S17 & 3 & 4 & 3 & & 3 & 9.83 \\
\hline S18 & 2 & 5 & 3 & & 3 & 39.46 \\
\hline S19 & 3 & 4 & 3 & & 3 & 10.63 \\
\hline S20 & 2 & 5 & 3 & & 3 & 50.48 \\
\hline S21 & 2 & 5 & 3 & & 3 & 46.23 \\
\hline S22 & 3 & 5 & 3 & & 3 & 8.19 \\
\hline S23 & 3 & 5 & 3 & & 3 & 8.80 \\
\hline S24 & 3 & 4 & 3 & & 3 & 35.31 \\
\hline \multicolumn{7}{|c|}{ Low/Medium Academic Effectiveness, Poor/Medium Quality pedagogy } \\
\hline S38 & 2 & 4 & 2 & & 1 & 6.93 \\
\hline S39 & 1 & 2 & 4 & & 3 & 63.38 \\
\hline S40 & 2 & 1 & 3 & & 3 & 9.53 \\
\hline S41 & 1 & 2 & 3 & & 3 & 55.05 \\
\hline S42 & 1 & 4 & 2 & & 2 & 29.61 \\
\hline S43 & 1 & 2 & 3 & & 3 & 4.16 \\
\hline S44 & 1 & 3 & 3 & & 3 & 4.8 \\
\hline S45 & 2 & 1 & 3 & & 3 & 43.05 \\
\hline S46 & 1 & 2 & 3 & & 3 & 42.57 \\
\hline S47 & 2 & 1 & 3 & & 3 & 5.97 \\
\hline S48 & 1 & 2 & 3 & & 3 & 30.70 \\
\hline S49 & 2 & 4 & 1 & & 2 & 9.20 \\
\hline
\end{tabular}

Continued

next 


\begin{tabular}{|c|c|c|c|c|c|}
\hline School & IEO & COS -5 & $\begin{array}{l}\text { Maths Residual } \\
\text { Score }\end{array}$ & $\begin{array}{l}\text { English } \\
\text { Residual Score }\end{array}$ & FSM \\
\hline \multicolumn{6}{|c|}{ Around Medium Academic Effectiveness, Medium Quality pedagogy } \\
\hline S34 & 2 & 2 & 3 & 3 & 7.07 \\
\hline S35 & 2 & 2 & 3 & 3 & 41.87 \\
\hline S33 & 2 & 4 & 3 & 3 & 35.44 \\
\hline S37 & 2 & 4 & 3 & 3 & 8.41 \\
\hline S50 & 3 & 2 & 3 & 3 & 25.08 \\
\hline S51 & 2 & 2 & 3 & 5 & 29.74 \\
\hline S52 & 1 & 4 & 3 & 3 & 17.53 \\
\hline S53 & 1 & 4 & 2 & 3 & 10.19 \\
\hline S54 & 2 & 4 & 2 & 3 & 17.74 \\
\hline S55 & 3 & 3 & 3 & 3 & 36.31 \\
\hline S56 & 3 & 2 & 2 & 3 & 3.33 \\
\hline S57 & 3 & 2 & 4 & 3 & 35.45 \\
\hline S58 & 3 & 3 & 3 & 3 & 25.55 \\
\hline S59 & 1 & 4 & 3 & 3 & 7.89 \\
\hline S60 & 3 & 3 & 3 & 3 & 14.86 \\
\hline S61 & 1 & 4 & 3 & 3 & 12.35 \\
\hline S62 & 2 & 2 & 4 & 4 & 29.41 \\
\hline S63 & 2 & 4 & 2 & 3 & 11.14 \\
\hline S64 & 1 & 4 & 4 & 3 & 43.05 \\
\hline \multicolumn{6}{|c|}{ No Category } \\
\hline S65 & 1 & 3 & 4 & 4 & 52.82 \\
\hline S66 & 1 & 5 & 3 & 2 & 11.19 \\
\hline S67 & 1 & 5 & 3 & 3 & 48.37 \\
\hline S68 & 1 & 3 & 5 & 3 & 15.27 \\
\hline S69 & 3 & 4 & 2 & 2 & 1.34 \\
\hline S70 & 1 & 5 & 2 & 4 & 27.61 \\
\hline S71 & 3 & 4 & 1 & 2 & 59.55 \\
\hline S72 & 1 & 5 & 3 & 3 & 23.01 \\
\hline
\end{tabular}

\begin{tabular}{|llll|}
\hline * & \multicolumn{3}{l|}{ Possible Scores on the IEO, COS-5, Maths and English Residual Scores } \\
\hline Measure & Possible Scores & Lowest Possible & Highest Possible \\
IEO & $1-3$ & 1 & 3 \\
COS-5 & $1-5$ & 1 & 5 \\
Maths/English Residual & $1-5$ & 1 & 5 \\
\hline
\end{tabular}




\section{Appendix 4 IEO and COS-5: Training and Reliability}

Training

The COS-5 and IEO instruments are complex requiring intensive training to prepare researchers for their use in Year 5 classrooms. Ten Research Assistants were recruited and all had extensive experience of working in primary schools both as practitioners and researchers.

A total of 12 days were needed to complete the training for both the IEO and COS-5 instruments, excluding time for researcher review of materials and classroom and video training. The training programme covered:

review of the documentation associated with the instruments

familiarisation with definitions

instructions on administration

video training on scoring

reviews of scoring

individual and paired observations in naturalistic settings.

Following initial in-house and video training, the researchers then tested the instrument in the field with both individual and paired observations. These observations were completed in Year 5 classrooms of schools unrelated to the project. At each stage of the training, researchers had to compare assessments and give justifications for their judgements. This was seen as an important part of validating the reliability of the observations. Both the COS- 5 and the IEO rely on numerical ratings and qualitative justifications.

Reliability

Checks on inter-observer reliability were conducted at each stage of the training in order to ensure consistency across coders. Final inter-observer reliability was achieved after the extensive training period. The format for reliability was video tapes of whole lessons which observers coded in isolation. This follows the procedures used by the NICHD for reliability. In EPPE 3-11 ten researchers coded eleven lessons for the COS-5 instrument and six lessons for the IEO. The lessons covered Literacy, Mathematics, Geography and Start of the afternoon.

For the COS-5 instrument inter-observer reliability was available from the NICHD study (reliability for the IEO was unavailable). The NICHD (2001) reported "average exact agreement with the gold-standard videotape test for the time-sampled codes, estimated by correlation with master-coders scores, was $.848 \ldots . . .$. average live reliability across all global ratings, estimated using correlations was .714" (NICHD, 2001, p 6). In the EPPE 3-11 reliability inter-rater agreement was also assessed using simple correlations for comparison with the NICHD data, exact agreement with the gold standard was $r=0.82-$ with a range of $0.75-0.87$, and average exact agreement across all raters was $r=0.80$ with a range of $0.68-0.95$.

In addition inter-observer agreement was assessed for each instrument using the Kappa statistics. Each observer was compared against a gold standard for each lesson type; nine pairs of comparisons were calculated for each lesson type within each instrument. The derived scores were then averaged across lessons and across observers for each instrument. Inter-observer agreement was high for both the COS-5 (weighted Kappa scores, 0.56 - 0.920; with a mean of 0.80) and the IEO (weighted Kappa scores, $0.55-0.84$ with a mean of 0.74 ) instruments. 


\section{Appendix 5.1 Organisation}

Quantitative Analysis

\begin{tabular}{|c|c|c|c|c|c|}
\hline \multirow[t]{2}{*}{ Measure } & \multicolumn{3}{|c|}{$\begin{array}{l}\text { Academic } \\
\text { Effectiveness (X) }\end{array}$} & \multirow[b]{2}{*}{$p$} & \multirow[t]{2}{*}{ Bonferroni } \\
\hline & Low & Med & High & & \\
\hline $\begin{array}{l}\text { CT's resources are well-organised, } \\
\text { managed and fit for purpose }(1-3, \max =3) \text {, } \\
F(2,230)=32.861\end{array}$ & 2.34 & 2.71 & 2.96 & .000 & $\begin{array}{l}\mathrm{L}<\mathrm{M}(.000) \\
\mathrm{L}<\mathrm{H}(.000) \\
\mathrm{M}<\mathrm{H}(.005)\end{array}$ \\
\hline $\begin{array}{l}\text { Productive use of instructional time }(1-7 \text {, } \\
\max =7), F(2,230)=45.940\end{array}$ & 3.56 & 4.83 & 5.77 & .000 & $\begin{array}{l}\mathrm{L}<\mathrm{M}(.000) \\
\mathrm{L}<\mathrm{H}(.000) \\
\mathrm{M}<\mathrm{H}(.000)\end{array}$ \\
\hline $\begin{array}{l}\text { CT is clear about what } \mathrm{s} \text { /he expects the } \\
\text { children to do in their activities }(1-5 \text {, max }= \\
5), F(2,230)=25.354\end{array}$ & 4.18 & 4.71 & 4.88 & .000 & $\begin{array}{l}\mathrm{L}<\mathrm{M}(.000) \\
\mathrm{L}<\mathrm{H}(.000) \\
\mathrm{M}=\mathrm{H}(.335)\end{array}$ \\
\hline $\begin{array}{l}\text { Time TC spends on transitions/ } \\
\text { management/ business }(0-10, \max =10) \text {, } \\
\mathrm{F}(2,230)=2.443\end{array}$ & 2.32 & 2.04 & 1.26 & .089 & $\begin{array}{l}\mathrm{L}=\mathrm{M}(1.000) \\
\mathrm{L}=\mathrm{H}(.101) \\
\mathrm{M}=\mathrm{H}(.384)\end{array}$ \\
\hline $\begin{array}{l}\text { Time CT spends on "business" }(0-10, \max \\
=10), F(2,230)=1.454\end{array}$ & 1.70 & 1.26 & 1.25 & .236 & $\begin{array}{l}\mathrm{L}=\mathrm{M}(.472) \\
\mathrm{L}=\mathrm{H}(.391) \\
\mathrm{M}=\mathrm{H}(1.000)\end{array}$ \\
\hline $\begin{array}{l}\text { TC's level of self-reliance }(1-7, \max =7) \text {, } \\
F(2,230)=7.887\end{array}$ & 3.90 & 4.10 & 4.69 & .000 & $\begin{array}{l}\mathrm{L}=\mathrm{M}(1.000) \\
\mathrm{L}<\mathrm{H}(.001) \\
\mathrm{M}<\mathrm{H}(.017)\end{array}$ \\
\hline $\begin{array}{l}\text { Children are responsible for time and } \\
\text { resources }(\max =3), F(2,230)=17.231\end{array}$ & 1.49 & 2.08 & 2.20 & .000 & $\begin{array}{l}\mathrm{L}<\mathrm{M}(.000) \\
\mathrm{L}<\mathrm{H}(.000) \\
\mathrm{M}=\mathrm{H}(1.000)\end{array}$ \\
\hline $\begin{array}{l}\text { Classroom routines }(\text { IEO) }(1-5, \max =5) \text {. } \\
F(2,55)=12.850\end{array}$ & 2.85 & 3.83 & 4.60 & .000 & $\begin{array}{l}\mathrm{L}<\mathrm{M}(.023) \\
\mathrm{L}<\mathrm{H}(.000) \\
\mathrm{M}=\mathrm{H}(.106)\end{array}$ \\
\hline
\end{tabular}

Qualitative descriptions

Some examples from the classroom observation instruments:

"No sense of time wasted." (School 1, COS-5 (2), p 5).

"Little time wasted. Children quickly settled to the task and a high percentage were on task" (School 3, COS-5 (3), p 3)

"Routines are very efficient - have monitors for everything to help the teacher. No disruption" (School 5, COS-5 (7), p 5)

"Well established routine" (School 6, COS-5 (1), p 5)

"From the very beginning a quick pace. No time lost whatsoever" ( School 1, Literacy IEO, p 3)

"Difficult to see how any more could have been squeezed in" (School 1, Numeracy IEO, p 3)

"Children immediately sit on carpet as arrive in room. Routine to brainstorm in pairs then as class went smoothly" (School 2, Literacy IEO, p 3)

"No time lost. Very quick start to Literacy ... Finally all cleared up quickly. They know what to do and they do it" (School 4, Literacy IEO, p 3)

"Children familiar with routines - they know what to do when they return from assembly" (School 6, Numeracy IEO, p 3)

"Couldn't be smoother" (School 7, Literacy IEO, p 3)

"A well oiled machine. Happy confident children move easily from one activity to the next" (School 7, Numeracy IEO, p 3)

"No time lost ever ... Great routines which all follow" (School 8, Literacy IEO, p 5) 


\section{Appendix 5.2 Shared Goals}

Quantitative Analysis

\begin{tabular}{|l|l|l|l|l|l|}
\hline \multirow{2}{*}{ Measure } & \multicolumn{2}{|l|}{$\begin{array}{l}\text { Academic } \\
\text { Effectiveness (X) }\end{array}$} & & \multirow{2}{*}{ Bonferroni } \\
\cline { 2 - 6 } & Low & Med & High & $\mathrm{p}$ & \\
\hline $\begin{array}{l}\text { The learning intentions of the lesson/ } \\
\text { activity are clear to all children (1-3, max }= \\
3), \mathrm{F}(2,230)=14.534\end{array}$ & & 2.65 & 2.85 & .000 & $\begin{array}{l}\mathrm{L}<\mathrm{M}(.017) \\
\mathrm{L}<\mathrm{H}(.000) \\
\mathrm{M}<\mathrm{H}(.046)\end{array}$ \\
\hline $\begin{array}{l}\text { Teacher ensures concepts/ideas are clear } \\
\text { to all (max }=5) \\
\mathrm{F}(2,230)=25.354\end{array}$ & 4.18 & 4.71 & 4.88 & .000 & $\begin{array}{l}\mathrm{L}<\mathrm{M}(.000) \\
\mathrm{L}<\mathrm{H}(.000) \\
\mathrm{M}=\mathrm{H}(.335)\end{array}$ \\
\hline
\end{tabular}

Qualitative descriptions

A good example of teachers not only checking that their pupils understood the key concepts in a lesson but modifying their lesson plan accordingly came from School 2. After an introduction to explain symmetry (the children had been working on this topic in Art as well as in Maths), the children were asked to work in pairs and create patterns for each other to repeat. The patterns had to contain two lines of symmetry and a perpendicular line. Despite a careful introduction to the task and modelling how to approach it, the children are still not sure what to do (there is much confusion over how to draw a perpendicular line). When the teacher realised that the confusion was wide-spread, she stopped the lesson, re-explained the task and then asked the children to have another go (School 2, Numeracy IEO). 


\section{Appendix 5.3 Classroom climate}

Quantitative Analysis

\begin{tabular}{|c|c|c|c|c|c|}
\hline \multirow[t]{2}{*}{ Measure } & \multicolumn{3}{|c|}{$\begin{array}{c}\text { Academic } \\
\text { Effectiveness }(\mathrm{X})\end{array}$} & & \multirow[t]{2}{*}{ Bonferroni } \\
\hline & Low & Med & High & $p$ & \\
\hline $\begin{array}{l}\text { Positive classroom climate, COS-5, (1-7, } \\
\max =7), F(2,230)=41.924\end{array}$ & 4.22 & 5.32 & 6.07 & .000 & $\begin{array}{l}\mathrm{L}<\mathrm{M}(.000) \\
\mathrm{L}<\mathrm{H}(.000) \\
\mathrm{M}<\mathrm{H}(.001)\end{array}$ \\
\hline $\begin{array}{l}\text { Negative classroom climate, COS-5, (1-7, } \\
\max =7), F(2,230)=19.190\end{array}$ & 2.59 & 1.49 & 1.35 & .000 & $\begin{array}{l}\mathrm{L}>\mathrm{M}(.000) \\
\mathrm{L}>\mathrm{H}(.000) \\
\mathrm{M}=\mathrm{H}(1.000)\end{array}$ \\
\hline $\begin{array}{l}\text { Teacher detachment, COS-5, }(1-7, \max = \\
7), F(230)=14.417\end{array}$ & 3.02 & 1.74 & 1.99 & .000 & $\begin{array}{l}\mathrm{L}>\mathrm{M}(.000) \\
\mathrm{L}>\mathrm{H}(.000) \\
\mathrm{M}=\mathrm{H}(.986)\end{array}$ \\
\hline $\begin{array}{l}\text { Classroom climate, IEO, }(1-5, \max =5) \text {, } \\
F(2,55)=13.741\end{array}$ & 3.10 & 3.94 & 4.75 & .000 & $\begin{array}{l}\mathrm{L}<\mathrm{M}(.035) \\
\mathrm{L}<\mathrm{H}(.000) \\
\mathrm{M}<\mathrm{H}(.047)\end{array}$ \\
\hline $\begin{array}{l}\text { Teacher displays positive affect, COS- } 5,(0- \\
10, \max =10), F(2,230)=.307\end{array}$ & 0.46 & 0.60 & 0.48 & .736 & $\begin{array}{l}L=M(1.000) \\
L=H(1.000) \\
M=H(1.000)\end{array}$ \\
\hline $\begin{array}{l}\text { Teacher displays negative affect, COS- } 5 \text {, } \\
(0-10, \max =10), F(2,230)=4.935\end{array}$ & 0.24 & 0.01 & 0.00 & .008 & $\begin{array}{l}\mathrm{L}>\mathrm{M}(.032) \\
\mathrm{L}>\mathrm{H}(.015) \\
\mathrm{M}=\mathrm{H}(1.000)\end{array}$ \\
\hline $\begin{array}{l}\text { Target child displays positive/neutral affect } \\
\text { towards peers, COS- } 5,(0-10, \text { max }=10) \text {, } \\
F(2,230)=.241\end{array}$ & 2.88 & 2.58 & 2.75 & .786 & $\begin{array}{l}L=M(1.000) \\
L=H(1.000) \\
M=H(1.000)\end{array}$ \\
\hline $\begin{array}{l}\text { Target child displays negative/aggressive } \\
\text { affect towards peers, COS- } 5,(0-10 \text {, max }= \\
10), F(2,230)=0.923\end{array}$ & 0.11 & 0.03 & 0.06 & .399 & $\begin{array}{l}L=M(.541) \\
L=H(1.000) \\
M=H(1.000)\end{array}$ \\
\hline $\begin{array}{l}\text { Target child displays positive affect towards } \\
\text { teacher, COS-5, }(0-10, \max =10), F(2,230) \\
=1.941\end{array}$ & 0.52 & 0.42 & 0.73 & .146 & $\begin{array}{l}\mathrm{L}=\mathrm{M}(1.000) \\
\mathrm{L}=\mathrm{H}(.593) \\
\mathrm{M}=\mathrm{H}(.166)\end{array}$ \\
\hline $\begin{array}{l}\text { Target child displays negative affect } \\
\text { towards teacher, COS }-5,(0-10, \text { max }=10) \text {, } \\
F(2,230)=1.936\end{array}$ & 0.02 & 0.00 & 0.00 & .147 & $\begin{array}{l}\mathrm{L}=\mathrm{M}(.288) \\
\mathrm{L}=\mathrm{H}(.259) \\
\mathrm{M}=\mathrm{H}(1.000)\end{array}$ \\
\hline $\begin{array}{l}\text { Child demonstrates positive affect, COS-5, } \\
(1-7, \max =7), F(2,230)=2.593\end{array}$ & 4.28 & 4.61 & 4.65 & .077 & $\begin{array}{l}\mathrm{L}=\mathrm{M}(.219) \\
\mathrm{L}=\mathrm{H}(.112) \\
\mathrm{M}=\mathrm{H}(1.000)\end{array}$ \\
\hline $\begin{array}{l}\text { Child-teacher relationship, COS-5, } \quad(1-7, \\
\max =7), F(2,230)=8.387\end{array}$ & 3.94 & 3.99 & 4.65 & .000 & $\begin{array}{l}\mathrm{L}=\mathrm{M}(1.000) \\
\mathrm{L}<\mathrm{H}(.001) \\
\mathrm{M}<\mathrm{H}(.003)\end{array}$ \\
\hline $\begin{array}{l}\text { Target child is sociable/cooperative with } \\
\text { peers, COS-5, }(1-7, \max =7), \mathrm{F}(2,230)= \\
4.026\end{array}$ & 3.50 & 3.61 & 4.30 & .019 & $\begin{array}{l}\mathrm{L}=\mathrm{M}(1.000) \\
\mathrm{L}=\mathrm{H}(.027) \\
\mathrm{M}=\mathrm{H}(.086)\end{array}$ \\
\hline $\begin{array}{l}\text { Children are well-liked and respected by } \\
\text { peers, COS-5, }(1-3, \max =3), F(2,230)= \\
29.747\end{array}$ & 2.19 & 2.61 & 2.78 & .000 & $\begin{array}{l}\mathrm{L}<\mathrm{M}(.000) \\
\mathrm{L}<\mathrm{H}(.000) \\
\mathrm{M}<\mathrm{H}(.010)\end{array}$ \\
\hline $\begin{array}{l}\text { Teacher sensitivity, COS-5, }(1-7, \max =7) \text {, } \\
F(2,230)=33.486\end{array}$ & 3.56 & 4.85 & 5.57 & .000 & $\begin{array}{l}\mathrm{L}<\mathrm{M}(.000) \\
\mathrm{L}<\mathrm{H}(.000) \\
\mathrm{M}<\mathrm{H}(.015)\end{array}$ \\
\hline $\begin{array}{l}\text { Social support for student learning, IEO, (1- } \\
5, \max =5), F(2,26)=6.293\end{array}$ & 3.00 & 4.22 & 4.30 & .000 & $\begin{array}{l}\mathrm{L}<\mathrm{M}(.022) \\
\mathrm{L}<\mathrm{H}(.011) \\
\mathrm{M}=\mathrm{H}(1.000)\end{array}$ \\
\hline
\end{tabular}


Affection shown by the teacher:

Some examples from the classroom observation instruments:

"(The teacher) is happy and jokey with the rest of the class. Lots of smiles are shared. Mentors different groups - praise-encouragement. Obvious affection seen" (School 2, COS-5 (6), p 6)

"Good relationships seen, affectionate" (School 5, COS-5 (5), p 5)

"Happy and relaxed children ... Children smile lots at the teacher - she has a lovely jokey attitude and obvious fondness for the children" (School 2, Literacy IEO, p 3)

"Obvious affection for children" (School 8, Numeracy IEO, p 3)

Encouragement:

Some examples from the classroom observation instruments:

"Teacher is very involved and aware of children. Lots of praise, e.g. "fantastic"' (School 4, COS-5 (3), p 5)

"Teacher praises the way they are all getting on with each other. (Target child) Cooperates all task all session" (School 5, COS-5 (3), p 4)

"The teacher was supportive and encouraging, smiled a lot." (School 1, Literacy IEO, field notes, $\mathrm{p}$ 4)

"The teacher established firm, friendly control and the children responded. They clearly enjoyed their learning and all were respectful. Lots of praise, encouragement for the children" (School 3, Numeracy IEO, p 3)

"Demeanour of teacher: relaxed, does not raise her voice, lots of smiles . . laughed with children at the news reader reports. Very proud of children's achievements." (School 5, Literacy IEO, p 3)

"Relaxed, well prepared. . . Never raised voice. Lots of smiles and pats on back to children" (School 5, Numeracy IEO, p 3)

"Good evidence of mutual support between children. Class teacher and teaching assistant are both encouraging and listen respectfully to children's suggestions" (School 6, Literacy IEO, p 3)

Safe, supportive environment:

Some examples from the classroom observation instruments:

"Warm supportive feeling. Children very content" (School 3, COS-5 (8), p 5)

"This classroom is a safe place to take risks" (School 7, COS-5 (7), p 5)

"Lovely atmosphere - children laugh and smile" (School 8, COS-5 (2), p 5)

"An experienced teacher, firm, friendly with high expectations." (School 1, Literacy IEO, p. 3)

"Firm but friendly and supportive teaching approach. . . The children feel very secure in terms of both learning and socially" (School 1, Numeracy IEO, p 3)

"Supportive approach with high expectations" (School 1, Numeracy IEO, field notes, p 3)

"The classroom seemed totally safe. The children were confident and comfortable and enjoying each other's company; also respectful towards the teacher who they obviously liked" (School 3, Literacy IEO, p 3)

"A very safe classroom, the children are secure and confident. The teacher maintains firm, friendly and supportive control. All respect each other - adults, children. The children are enjoying their learning. Nothing less than a 5 [maximum score]) (School 4, Literacy IEO, $p$ 3)

"A lot of support for the children from the class teacher, the special needs teacher and the learning support assistant. Children were also supportive to each other." (School 4, Literacy IEO, p 3)

"A safe place to make mistakes" (School 7, Numeracy IEO, p 3) 
Mutual respect:

Some examples from the classroom observation instruments:

"A feeling that children were enjoying their learning and confident. A feeling of respect between children and teacher . . . Very aware of children. A very warm supportive attitude, which reflects a child-centred approach. No need to discipline." (School 1, COS-5 (1), p. 5)

"Genuine respect by children and teacher for all in the class" (School 6, COS-5 (4), p 5)

"Classroom climate ... a strong feeling of respect for each other. A mature attitude." (School 1, Literacy IEO, p 5)

"All (classroom climate) characterised by mutual respect" (School 4, Numeracy IEO, p 3)

"Such mutual respect allows children to confidently share their writing with the class.

Everyone listens intently as others read. Class teacher makes positive comments - but also some fair criticisms well received" (School 7, Literacy IEO, p 3)

"Respect obvious. Work well in groups - negotiate roles, share work and help others " (School 8, Literacy IEO, p 5) 


\section{Appendix 5.4 Behaviour Management}

Quantitative Analysis

\begin{tabular}{|c|c|c|c|c|c|}
\hline \multirow[t]{2}{*}{ Measure } & \multicolumn{3}{|c|}{$\begin{array}{l}\text { Academic } \\
\text { Effectiveness (X) }\end{array}$} & \multirow[b]{2}{*}{$p$} & \multirow[t]{2}{*}{ Bonferroni } \\
\hline & Low & Med & High & & \\
\hline $\begin{array}{l}\text { CT disciplines }(0-10, \max =10) \\
F(2,230)=8.230\end{array}$ & 0.55 & 0.14 & 0.12 & .000 & $\begin{array}{l}\mathrm{L}>\mathrm{M}(.003) \\
\mathrm{L}>\mathrm{H}(.001) \\
\mathrm{M}=\mathrm{H}(1.000)\end{array}$ \\
\hline $\begin{array}{l}\text { TC's general level of disruptive behaviour } \\
(\max =10) \\
F(2,230)=3.402\end{array}$ & 0.16 & .00 & .00 & .035 & $\begin{array}{l}\mathrm{L}>\mathrm{M}(.083) \\
\mathrm{L}>\mathrm{H}(.070) \\
\mathrm{M}=\mathrm{H}(1.000)\end{array}$ \\
\hline $\begin{array}{l}\text { TC's disruptiveness }(\max =7) \\
\mathrm{F}(2,230)=3.605\end{array}$ & 1.65 & 1.17 & 1.52 & .029 & $\begin{array}{l}\mathrm{L}>\mathrm{M}(.029) \\
\mathrm{L}=\mathrm{H}(1.000) \\
\mathrm{M}=\mathrm{H}(.173)\end{array}$ \\
\hline $\begin{array}{l}\text { Child is engaged in learning, COS-5, }(0-10 \text {, } \\
\max =10), F(2,230)=3.266\end{array}$ & 7.41 & 8.32 & 8.10 & .040 & $\begin{array}{l}\mathrm{L}<\mathrm{M}(.048) \\
\mathrm{L}=\mathrm{H}(.180) \\
\mathrm{M}=\mathrm{H}(1.000)\end{array}$ \\
\hline $\begin{array}{l}\text { Student engagement, IEO, }(1-5, \max =5) \text {, } \\
F(2,55)=9.640\end{array}$ & 3.15 & 3.94 & 4.50 & .000 & $\begin{array}{l}\mathrm{L}<\mathrm{M}(.046) \\
\mathrm{L}<\mathrm{H}(.000) \\
\mathrm{M}=\mathrm{H}(.257)\end{array}$ \\
\hline $\begin{array}{l}\text { Over control }(\max =7) \\
F(2,230)=4.269\end{array}$ & 2.84 & 2.06 & 2.31 & .015 & $\begin{array}{l}\mathrm{L}>\mathrm{M}(.015) \\
\mathrm{L}=\mathrm{H}(.146) \\
\mathrm{M}=\mathrm{H}(1.000)\end{array}$ \\
\hline $\begin{array}{l}\text { Chaos }(\max =7) \\
F(2,230)=11.279\end{array}$ & 2.30 & 1.43 & 1.51 & .000 & $\begin{array}{l}L>M(.000) \\
L>H(.000) \\
M=H(1.000)\end{array}$ \\
\hline
\end{tabular}

Qualitative descriptions

Some examples from the classroom observation instruments:

"Follows expectations and rules" (School 1, COS-5 (1), p 4)

"The whole class and all children are on task, but control is established by involving children in their learning" (School 1, COS-5 (1), p 5)

"The teacher was very aware of all that was happening in the class. No need for any discipline." (School 1, COS-5 (2), p 5)

"There was no sense of over-control" (School 1, COS-5 (3), p 5)

"The teacher uses his name several times - compliments him on his eagerness to volunteer and participate. Praises for correct answers. He smiles back at her several times. Eye contact." (School 2, COS-5 (1), p 5)

"Teacher has to discipline him once. Does so in jokey manner by saying all he will remember is the colour of the carpet" (School 2, COS-5 (5), p 5)

"Calm, quiet and firm discipline - mainly by expectation" (School 3, COS-5 (6), p 5)

"No disciplinary incidents, clear expectations. Children on task" (School 4, COS-5 (3), p 5)

"No chaos/poor discipline etc. Occasional quiet reminder of rules. All well organised" (School 4, COS-5 (8), p 5)

"No disruption. No discipline needed" (School 5, COS-5 (7), p 5)

"You can hear a pin drop in this class as children work out factors of 42 - and such a low buzz of enthusiasm" (School 7, COS-5 (7), p 5)

"The teacher has a 'strong' presence and maintains a high profile throughout . . . Feeling of teacher and children jointly seeking progress. Control by expectation, involvement in learning. Teacher is firm and friendly" (School 1, Literacy IEO, p 3)

"In whole session, no disciplinary problems. Only one very minor disagreement by 3 boys which they settled amicably" (School 3, Literacy IEO, p 3) 
"The teacher established firm friendly control and the children responded" (School 3, Numeracy IEO, p 4)

"The teacher maintains firm, friendly and supportive control" (School 4, Literacy IEO, p 3)

"Teacher experienced and in control. Talks at normal volume, children listen attentively. She allows lots of quiet on task discussion in pairs and with whole class - children were on task, no disruptions observed" (School 5, Numeracy IEO, p 3)

"High expectations, challenging work, appropriate and qualified praise - and no chance to slack" (School 7, Literacy IEO, p 3)

"No discipline problems. All on task and enthusiastic about all of lesson" (School 8, Literacy IEO, p 3) 


\section{Appendix 5.5 Collaborative learning}

Quantitative analysis

\begin{tabular}{|c|c|c|c|c|c|}
\hline \multirow{2}{*}{ Measure } & \multicolumn{3}{|c|}{$\begin{array}{l}\text { Academic } \\
\text { Effectiveness }(X)\end{array}$} & \multirow[b]{2}{*}{$p$} & \multirow{2}{*}{ Bonferroni } \\
\hline & Low & Med & High & & \\
\hline $\begin{array}{l}\text { Target child works in a collaborative group } \\
(\max =10) \\
F(2,230)=2.249\end{array}$ & 0.46 & 0.14 & 0.70 & .108 & $\begin{array}{l}\mathrm{L}=\mathrm{M}(.683) \\
\mathrm{L}=\mathrm{H}(1.000) \\
\mathrm{M}=\mathrm{H}(.106)\end{array}$ \\
\hline $\begin{array}{l}\text { Target child is involved in collaborative } \\
\text { work }(0-10, \max =10) \\
F(2,230)=1.397\end{array}$ & 0.79 & 0.32 & 0.72 & .249 & $\begin{array}{l}\mathrm{L}=\mathrm{M}(.358) \\
\mathrm{L}=\mathrm{H}(1.000) \\
\mathrm{M}=\mathrm{H}(.556)\end{array}$ \\
\hline
\end{tabular}

\begin{tabular}{|l|l|c|c|c|}
\hline \multirow{2}{*}{ Measure } & $\begin{array}{l}\text { Academic Effectiveness / } \\
\text { Quality of Pedagogy }\end{array}$ & No & Yes & Total \\
\hline \multirow{2}{*}{ IEO: Group work seen (yes or no) } & Low / Poor & 9 & 7 & 16 \\
\cline { 2 - 5 } & Medium & 9 & 7 & 16 \\
\cline { 2 - 5 } & High / Good & 8 & 6 & 14 \\
\hline
\end{tabular}

* Two observations per school. Totals are smaller than twice the total number of schools in each group as this measure was taken during the second year of observations only.

Qualitative descriptions

Some examples from the classroom observation instruments:

"Worked closely with peers, discussing work and approach to the problem" (School 1, COS$5(1), \mathrm{p} 4)$

"Took a leading role when working with peers" (School 1, COS-5 (2), p 4)

"Children were working collaboratively. The classroom was a buzz of activity" (School 1, COS-5 (2), p 5)

"Collaborative work, children involved" (School 1, COS-5 (3), p 5)

"Talking, working with peers. At times ... (the child) takes the lead in discussion" (School 1, COS-5 (4), p 4)

"A collaborative situation" (School 1, COS-5 (5), p 4)

"Cooperates well in group reading - take turns in group, no arguments. Listens well to peers read" (School 2, COS-5 (8), p 4)

Some examples from the classroom observation instruments:

"Clear evidence of good positive relationship with peers with 'response' partner and others on the table" (School 1, COS-5 (3), p 4)

"Children worked with response partners" (School 1, COS-5 (3), p 5)

"During the collaborative part, discusses well with partner" (School 5, COS-5 (1), p 4)

"Children participate ... read and discuss their work" (School 5, COS-5 (3), p 6)

"Children made excellent progress in using and applying their knowledge of place value in this whole class activity. Good teaching - and opportunities to learn from contributions of others" (School 7, COS-5 (2), p 5)

"Children are encouraged to discuss with their partner during the mental introduction to the lesson to decide on the 2 step process needed to multiply by 20" (School 2, Numeracy IEO, p 3) 


\section{Appendix 5.6 Personalised Learning}

Quantitative Analysis

\begin{tabular}{|l|l|l|l|l|l|}
\hline \multirow{2}{*}{ Measure } & \multicolumn{2}{|l|}{$\begin{array}{l}\text { Academic } \\
\text { Effectiveness }(\mathrm{X})\end{array}$} & & \multirow{2}{*}{ Bonferroni } \\
\cline { 2 - 5 } & Low & Med & High & $\mathrm{p}$ & \\
\hline $\begin{array}{l}\text { Teacher detachment }(1-7, \max =7) \\
\mathrm{F}(2,230)=14.417\end{array}$ & 3.02 & 1.74 & 1.99 & .000 & $\begin{array}{l}\mathrm{L}>\mathrm{M}(.000) \\
\mathrm{L}>\mathrm{H}(.000) \\
\mathrm{M}=\mathrm{H}(.986)\end{array}$ \\
\hline $\begin{array}{l}\text { Teacher sensitivity (1-7, max }=7) \\
\mathrm{F}(2,230)=33.486\end{array}$ & 3.56 & 4.85 & 5.57 & .000 & $\begin{array}{l}\mathrm{L}<\mathrm{M}(.000) \\
\mathrm{L}<\mathrm{H}(.000) \\
\mathrm{M}<\mathrm{H}(.015)\end{array}$ \\
\hline $\begin{array}{l}\text { Social support for student learning (IEO) (1- } \\
\text { 5, max }=5), \mathrm{F}(2,26)=6.293\end{array}$ & 3.00 & 4.22 & 4.30 & .006 & $\begin{array}{l}\mathrm{L}<\mathrm{M}(.022) \\
\mathrm{L}<\mathrm{H}(.011) \\
\mathrm{M}=\mathrm{H}(1.000)\end{array}$ \\
\hline $\begin{array}{l}\text { Richness and variety of instructional } \\
\text { material }(1-7, \max =7) \\
\mathrm{F}(2,230)=33.396\end{array}$ & 3.04 & 3.82 & 4.93 & .000 & $\begin{array}{l}\mathrm{L}<\mathrm{M}(.004) \\
\mathrm{L}<\mathrm{H}(.000) \\
\mathrm{M}<\mathrm{H}(.000)\end{array}$ \\
\hline
\end{tabular}

Qualitative descriptions

Some examples from the classroom observation instruments:

"Children were involved in their learning and enjoying the experience. Indicating teacher sensitivity and understanding of children's needs (School 1, COS-5 (3), p 5)

"Clearly knows children well. Gives sensitive support and realistic feedback as she moves round the room" (School 6, COS-5 (1), p 5)

"Teacher is very aware of children's strengths and weaknesses and gives appropriate support and asks questions to suit those she chooses to answer, often naming them rather than choosing volunteers" (School 7, COS-5 (2), p. 5)

"Knows children well. Directs some questions - and frames some for particular children" (School 7, COS-5 (7), p 5)

"Sure of her understanding of the children, their levels and needs. Also in her ability to extend appropriately." (School 1, Literacy IEO, p 3)

"Teacher encourages all to contribute ideas throughout. Children listen well to each other.

Teacher uses children's names to get them to contribute." (School 2, Literacy IEO, p 3)

"Teacher says to all about boy at front 'Very good boy spending his time reading whilst he's waiting.' This is one of very shy boys, less confident. She boosts his confidence here" (School 5, Literacy IEO field notes, p 1) 


\section{Appendix 5.7 Making Links Explicit}

\begin{tabular}{|c|c|c|c|c|c|}
\hline \multirow[t]{2}{*}{ Measure } & \multicolumn{3}{|c|}{$\begin{array}{l}\text { Academic } \\
\text { Effectiveness (X) }\end{array}$} & \multirow[b]{2}{*}{$p$} & \multirow[t]{2}{*}{ Bonferroni } \\
\hline & Low & Med & High & & \\
\hline $\begin{array}{l}\text { Cross-disciplinary connections, IEO }(1-5 \text {, } \\
\max =5), F(255,)=3.578\end{array}$ & 1.45 & 1.06 & 1.95 & .035 & $\begin{array}{l}\mathrm{L}=\mathrm{M}(.737) \\
\mathrm{L}=\mathrm{H}(.396) \\
\mathrm{M}<\mathrm{H}(.031)\end{array}$ \\
\hline $\begin{array}{l}\text { Linkage to life beyond the classroom, IEO, } \\
(1-5, \max =5) \\
\mathrm{F}(2,55)=.894\end{array}$ & 1.85 & 2.11 & 2.40 & .415 & $\begin{array}{l}\mathrm{L}=\mathrm{M}(1.000) \\
\mathrm{L}=\mathrm{H}(.561) \\
\mathrm{M}=\mathrm{H}(1.000)\end{array}$ \\
\hline
\end{tabular}

Qualitative descriptions

Some examples from the classroom observation instruments:

"A practical science session investigating sweeteners ... Totally absorbed all children" (School 1, COS-5 (4), p 5)

Lesson on seed dispersal includes pots of planted seeds, some of which are growing (School 2, COS-5 (6), p 5)

"Discuss symmetry in natural life, i.e. butterflies. The children have been studying symmetry in Art and in artists' work. Artwork is displayed and during Numeracy, a child points out the lines of symmetry" (School 2, Numeracy IEO, p 3)

"Children were dissecting flowers, sticking them in their books, recording, using magnifying glasses and microscopes. Engaged children" (School 3, COS-5 (6), p 5)

"Used children's experiences (about transport) in their writing " (School 4, COS-5 (2), p 5) 


\section{Appendix 5.8 Dialogic Teaching and Learning}

\begin{tabular}{|c|c|c|c|c|c|}
\hline \multirow[t]{2}{*}{ Measure } & \multicolumn{3}{|c|}{$\begin{array}{l}\text { Academic } \\
\text { Effectiveness (X) }\end{array}$} & \multirow[b]{2}{*}{$p$} & \multirow[t]{2}{*}{ Bonferroni } \\
\hline & Low & Med & High & & \\
\hline $\begin{array}{l}\text { Target child engaged in comprehension in } \\
\text { literacy, COS }-5,(1-10, \max =10) \\
F(2,230)=0.062\end{array}$ & 3.95 & 3.53 & 3.38 & .940 & $\begin{array}{l}L=M(1.000) \\
L=H(1.000) \\
M=H(1.000)\end{array}$ \\
\hline $\begin{array}{l}\text { Target child engaged in concept } \\
\text { development or problem solving in } \\
\text { numeracy, COS-5, }(1-10, \max =10) \\
F(2,230)=0.872\end{array}$ & 0.79 & 1.57 & 1.33 & .420 & $\begin{array}{l}\mathrm{L}=\mathrm{M}(.349) \\
\mathrm{L}=\mathrm{H}(.773) \\
\mathrm{M}=\mathrm{H}(1.000)\end{array}$ \\
\hline $\begin{array}{l}\text { Target child learning or performing analysis } \\
\text { or inference, COS-5, }(1-10, \max =10) \\
\mathrm{F}(2,230)=2.390\end{array}$ & 2.78 & 2.32 & 3.96 & .001 & $\begin{array}{l}\mathrm{L}=\mathrm{M}(1.000) \\
\mathrm{L}=\mathrm{H}(.096) \\
\mathrm{M}<\mathrm{H}(.012)\end{array}$ \\
\hline $\begin{array}{l}\text { Teacher teaching analysis, inference or } \\
\text { planning, COS-5, }(1-10, \max =10) \\
F(2,230)=7.030\end{array}$ & 1.48 & 1.72 & 2.17 & $.094^{*}$ & $\begin{array}{l}\mathrm{L}=\mathrm{M}(1.000) \\
\mathrm{L}=\mathrm{H}(.328) \\
\mathrm{M}=\mathrm{H}(.940)\end{array}$ \\
\hline $\begin{array}{l}\text { Reading as meaning making, IEO, }(1-5 \text {, } \\
\text { max }=5) \\
F(2,15)=1.808\end{array}$ & 3.33 & 3.43 & 4.60 & .198 & $\begin{array}{l}\mathrm{L}=\mathrm{M}(1.000) \\
\mathrm{L}=\mathrm{H}(.319) \\
\mathrm{M}=\mathrm{H}(.364)\end{array}$ \\
\hline $\begin{array}{l}\text { Higher order thinking in writing, IEO, }(1-5 \text {, } \\
\max =5) \\
F(2,26)=1.382\end{array}$ & 3.00 & 3.78 & 4.00 & .269 & $\begin{array}{l}\mathrm{L}=\mathrm{M}(.720) \\
\mathrm{L}=\mathrm{H}(.373) \\
\mathrm{M}=\mathrm{H}(1.000)\end{array}$ \\
\hline $\begin{array}{l}\text { Instructional conversations (literacy), IEO, } \\
(1-5, \max =5) \\
\mathrm{F}(2,26)=5.035\end{array}$ & 3.00 & 4.33 & 4.50 & .014 & $\begin{array}{l}\mathrm{L}<\mathrm{M}(.055) \\
\mathrm{L}<\mathrm{H}(.022) \\
\mathrm{M}=\mathrm{H}(1.000)\end{array}$ \\
\hline $\begin{array}{l}\text { Use of maths analysis, IEO, }(1-5, \max =5) \\
\mathrm{F}(2,26)=12.267\end{array}$ & 1.90 & 3.56 & 3.90 & .000 & $\begin{array}{l}\mathrm{L}<\mathrm{M}(.003) \\
\mathrm{L}<\mathrm{H}(.000) \\
\mathrm{M}=\mathrm{H}(1.000)\end{array}$ \\
\hline $\begin{array}{l}\text { Depth of knowledge and student } \\
\text { understanding (maths), IEO, }(1-5, \max =5) \\
F(2,25)=9.899\end{array}$ & 2.11 & 3.67 & 3.80 & .001 & $\begin{array}{l}\mathrm{L}<\mathrm{M}(.004) \\
\mathrm{L}<\mathrm{H}(.001) \\
\mathrm{M}=\mathrm{H}(1.000)\end{array}$ \\
\hline $\begin{array}{l}\text { Maths discourse and communication, IEO, } \\
(1-5, \max =5) \\
\mathrm{F}(2,26)=18.469\end{array}$ & 1.90 & 3.56 & 4.00 & .000 & $\begin{array}{l}\mathrm{L}<\mathrm{M}(.000) \\
\mathrm{L}<\mathrm{H}(.000) \\
\mathrm{M}=\mathrm{H}(.732)\end{array}$ \\
\hline $\begin{array}{l}\text { Locus of maths authority, IEO, }(1-5, \text { max }= \\
5) \\
F(2,26)=7.800\end{array}$ & 1.90 & 3.33 & 3.80 & .002 & $\begin{array}{l}\mathrm{L}<\mathrm{M}(.029) \\
\mathrm{L}<\mathrm{H}(.002) \\
\mathrm{M}=\mathrm{H}(1.000)\end{array}$ \\
\hline
\end{tabular}

Approaches significance

Some examples from the classroom observation instruments:

"Hum of working conversation, all learning based. Children were positive and were enjoying their learning" (School 1, COS-5 (3), p 5)

"The teacher discusses choice books in a friendly manner - respects the child's opinion re: their choice" (School 2, COS-5 (7), p 5)

"High expectations from the teacher and she gave lots of encouragement, challenging children to succeed. Discussion between teachers and children at class, group and individual levels ... A A high level of appropriate support for children's learning" (School 3, Numeracy IEO, p 3)

"The introduction on the carpet fully engaged the children in their learning and there was considerable evidence of shared understanding ... The teacher used challenging questions to draw out children's understanding" (School 4, Numeracy IEO, p 4)

The teacher asks the children "Why is this (real life problems) important?" (School 5, 
Numeracy IEO, field notes, $\mathrm{p}$ 2)

When reading a poem to her pupils, the School 8 teacher "stops regularly to question about inference" and then questions the children about the way the verses were written (as opposed to just asking about their content), (School 8, Literacy IEO, lesson plan p 1) 


\section{Appendix 5.9 Assessment for learning}

\begin{tabular}{|l|l|l|l|l|l|}
\hline \multirow{2}{*}{ Measure } & \multicolumn{2}{|l|}{$\begin{array}{l}\text { Academic } \\
\text { Effectiveness (X) }\end{array}$} & & \multirow{2}{*}{ Bonferroni } \\
\cline { 2 - 5 } & Low & Med & High & $\mathrm{p}$ & \\
\hline $\begin{array}{l}\text { Evaluative feedback, COS-5 (1-7, max }=7) \\
\mathrm{F}(2,230)=11.237\end{array}$ & 3.20 & 3.90 & 4.42 & .000 & $\begin{array}{l}\mathrm{L}<\mathrm{M}(.026) \\
\mathrm{L}<\mathrm{H}(.000) \\
\mathrm{M}=\mathrm{H}(.158)\end{array}$ \\
\hline $\begin{array}{l}\text { Children could reflect on their learning } \\
\text { through review, COS-5, (1-3, max = 3) } \\
\mathrm{F}(2,230)=12.677\end{array}$ & 1.79 & 1.72 & 2.20 & .000 & $\begin{array}{l}\mathrm{L}=\mathrm{M}(1.000) \\
\mathrm{L}<\mathrm{H}(.000) \\
\mathrm{M}<\mathrm{H}(.000)\end{array}$ \\
\hline
\end{tabular}

Some examples from the classroom observation instruments:

"Feedback at the individual level and also at class level; there was depth" (School 1, COS-5 (2), p 5)

"The teacher was involved with children throughout - as a whole class or with groups or individuals. Responded accordingly . . . every indication of good quality feedback" (School 1, COS-5 (3), p 5)

"Uses children's writing to model and feedback. Uses children's paragraph structures as basis for work (School 3, COS-5 (3), p 5)

"The teacher uses children's answers to model correct answers" (School 5, COS-5 (7), p 5)

"Gives individual feedback to most children as moves round" (School 6, COS-5 (1), p 5)

"Children are encouraged to evaluate their own handwriting. The class teacher also gives

feedback as she goes round the class discussing handwriting" (School 7, COS-5 (1), p 5) 


\section{Appendix 5.10 Plenary}

\begin{tabular}{|c|c|c|c|c|c|}
\hline \multicolumn{6}{|c|}{ Plenary * Effectiveness Crosstabulation } \\
\hline \multicolumn{6}{|c|}{ Count \begin{tabular}{l|l|l|l} 
& & &
\end{tabular}} \\
\hline & & \multicolumn{4}{|c|}{ Effectiveness } \\
\hline & & Low & Medium & High & Total \\
\hline \multirow[t]{3}{*}{ Plenary } & 0 & 12 & 7 & 7 & 26 \\
\hline & 1 & 4 & 9 & 7 & 20 \\
\hline & Total & 16 & 16 & 14 & 46 \\
\hline
\end{tabular}


Ref: DFE-RR129

ISBN: 978-1-84775-937-5

(C) Institute of Education, Birkbeck (University of London) and University of Oxford

June 2011 